\title{
Flat morphisms of finite presentation are very flat
}

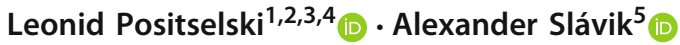

Received: 1 February 2019 / Accepted: 11 September 2019 / Published online: 25 September 2019 (c) Fondazione Annali di Matematica Pura ed Applicata and Springer-Verlag GmbH Germany, part of Springer Nature 2019

\begin{abstract}
Principal affine open subsets in affine schemes are an important tool in the foundations of algebraic geometry. Given a commutative ring $R, R$-modules built from the rings of functions on principal affine open subschemes in Spec $R$ using ordinal-indexed filtrations and direct summands are called very flat. The related class of very flat quasi-coherent sheaves over a scheme is intermediate between the classes of locally free and flat sheaves, and has serious technical advantages over both. In this paper, we show that very flat modules and sheaves are ubiquitous in algebraic geometry: if $S$ is a finitely presented commutative $R$-algebra which is flat as an $R$-module, then $S$ is a very flat $R$-module. This proves a conjecture formulated in the February 2014 version of the first author's long preprint on contraherent cosheaves (Positselski in Contraherent cosheaves, arXiv:1209.2995 [math.CT]). We also show that the (finite) very flatness property of a flat module satisfies descent with respect to commutative ring homomorphisms of finite presentation inducing surjective maps of the spectra.
\end{abstract}

Keywords Commutative rings - Finitely presented commutative algebras - Flat modules · Flat morphisms of schemes - Very flat modules · Very flat morphisms of schemes · Finitely very flat modules · Contraadjusted modules $\cdot$ Contramodules $\cdot$ Cotorsion theories · Approximation sequences · Surjective descent

Mathematics Subject Classification 14B25 $13 \mathrm{~B} 30 \cdot 13 \mathrm{~J} 10 \cdot 13 \mathrm{C} 60 \cdot 13 \mathrm{D} 99$

$凶 \quad$ Leonid Positselski

positselski@yandex.ru

Alexander Slávik

Slavik.Alexander@seznam.cz

1 Institute of Mathematics of the Czech Academy of Sciences, Žitná 25, 11567 Prague 1, Czech Republic

2 Laboratory of Algebraic Geometry, National Research University Higher School of Economics, Moscow, Russia 119048

3 Laboratory of Algebra and Number Theory, Institute for Information Transmission Problems, Moscow, Russia 127051

4 Department of Mathematics, Faculty of Natural Sciences, University of Haifa, 31905 Mount Carmel, Haifa, Israel

5 Department of Algebra, Faculty of Mathematics and Physics, Charles University, Sokolovská 83, 186 75 Prague 8, Czech Republic 


\section{Contents}

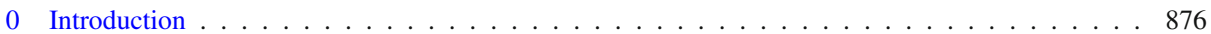

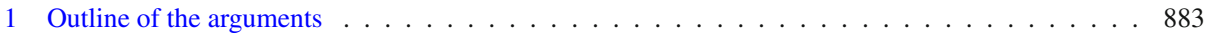

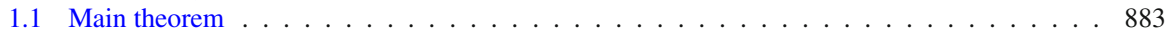

1.2 Noetherian main lemma . . . . . . . . . . . . . . . . . . . . . 883

1.3 Finitely very flat main lemma . . . . . . . . . . . . . . . . . . . . 883

1.4 Bounded torsion main lemma . . . . . . . . . . . . . . . . . . . . . . 884

1.5 Obtaining contraadjusted modules $\ldots \ldots \ldots \ldots \ldots \ldots$

1.6 Toy Main Lemma . . . . . . . . . . . . . . . . . . . . . . . . . . . . 885

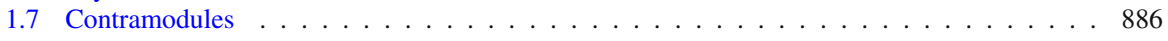

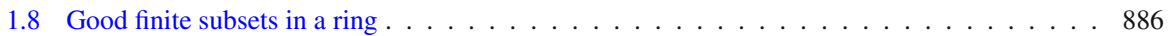

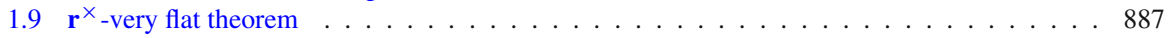

1.10 Flat lemma . . . . . . . . . . . . . . . . . . . . . . . . . . 887

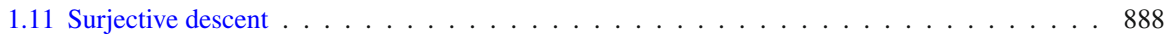

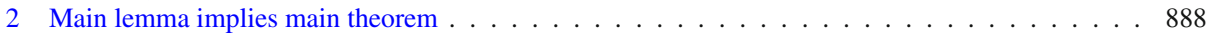

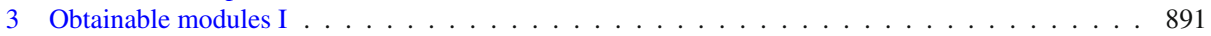

4 Toy examples of a main lemma . . . . . . . . . . . . . . . . . . . . . . 893

5 Contramodule approximation sequences . . . . . . . . . . . . . . . . . . . . 897

5.1 Cotorsion theories in abelian categories _ . . . . . . . . . . . . . . . . . . 897

5.2 Flat cotorsion theory in $r$-contramodule $R$-modules for a Noetherian commutative ring $R \quad$. 898

5.3 Very flat cotorsion theory in the bounded torsion case . . . . . . . . . . . . . . 900

5.4 Separated $r$-contramodule $R$-modules ～. . . . . . . . . . . . . . . . . . . 903

5.5 Quotseparated $r$-contramodule $R$-modules . . . . . . . . . . . . . . . . . . . . 904

5.6 Very flat cotorsion theory in quotseparated $r$-contramodule $R$-modules . . . . . . . . . 906

6 Obtainable modules II . . . . . . . . . . . . . . . . . . . . . . . . . . . . . . . . 909

7 Noetherian Main Lemma . . . . . . . . . . . . . . . . . . . . . . . . . . . . . . . 910

8 Finitely very flat Main Lemma $\ldots \ldots \ldots 12 \ldots \ldots \ldots$

9 Examples and applications . . . . . . . . . . . . . . . . . . . . . . . 917

10 Descent for surjective morphisms . . . . . . . . . . . . . . . . . . . . . . . 920

References . . . . . . . . . . . . . . . . . . . . . . 923

\section{Introduction}

0.1. The abelian category of modules over a ring has enough projective objects, which can be used in order to construct left derived functors. Right derived functors can be constructed using injective modules.

The abelian category of quasi-coherent sheaves on a scheme also has enough injective objects but, generally speaking, no projectives. On a quasi-projective scheme, there are enough invertible sheaves, and some other schemes may have enough locally free sheaves, of finite or infinite rank.

Locally free sheaves of possibly infinite rank, or what is the same over a locally Noetherian scheme [1, Corollary 4.5], locally projective sheaves, actually form a quite remarkable class. In particular, the projectivity of a module over a commutative ring is a local property, satisfying descent with respect to Zariski coverings, and in fact, even with respect to faithfully flat ring morphisms $[17,28]$. But what if there are not enough locally projective sheaves?

A Noetherian scheme is said to have the resolution property if it has enough locally free sheaves of finite rank [31]. It seems to be an open question whether there exist algebraic varieties that do not have the resolution property.

On the other hand, on any quasi-compact semi-separated scheme there are enough flat quasi-coherent sheaves [13, Section 2.4], [5, Lemma A.1]. But arbitrary flat modules over a commutative ring are much more complicated, from the homological point of view, than the projective modules. In particular, flat modules over a ring that is not necessarily Noetherian 
of finite Krull dimension form an exact category of infinite homological dimension.

0.2. In fact, the derived category of the exact category of flat modules over an associative ring was studied and shown to be equivalent to the homotopy category of projective modules [15]. Extending this approach to schemes, one can define the mock homotopy category of (nonexistent) projective quasi-coherent sheaves on a quasi-compact semi-separated scheme as the derived category of the exact category of flat quasi-coherent sheaves [14].

One of the potential shortcomings of this approach is that it is not directly applicable to matrix factorizations. Matrix factorizations are a kind of curved differential modules, so the conventional construction of the derived category does not make sense for them. Instead, one has to use derived categories of the second kind, such as the coderived and the absolute derived categories [5,16]. However, for an exact category of infinite homological dimension, the coderived category may differ from the derived category [19, Section 2.1], [20, Examples 3.3].

One of the approaches suggested in [5, Remark 2.6] is to use the very flat quasi-coherent sheaves (which were previously defined in [22, Section 4.1]) in lieu of the locally free or flat ones. The aim of this paper is to show just how well this approach turns out to work.

0.3. For every quasi-compact semi-separated scheme $X$, there is a full subcategory of very flat quasi-coherent sheaves $X-\mathrm{qcoh}_{\mathrm{vfl}} \subset X$-qcoh in the abelian category $X$-qcoh of quasicoherent sheaves on $X$ having the following properties:

(VF1) All very flat quasi-coherent sheaves are flat, that is $X-$ qcoh $_{\mathrm{vfl}} \subset X-\mathrm{qcoh}_{\mathrm{fl}}$, where $X-$ qcoh $_{\mathrm{fl}} \subset X$-qcoh denotes the full subcategory of flat quasi-coherent sheaves on $X$.

(VF2) All locally projective quasi-coherent sheaves are very flat, that is $X$-qcoh $X$-qcoh ${ }_{\mathrm{vfl}}$, where $X$-qcoh , $_{\mathrm{p}} \subset X$-qcoh denotes the full subcategory of locally projective quasi-coherent sheaves on $X$.

(VF3) The tensor product of any two very flat quasi-coherent sheaves is very flat, that is $X-\mathrm{qcoh}_{\mathrm{vfl}} \otimes_{\mathcal{O}_{X}} X-\mathrm{qcoh}_{\mathrm{vfl}} \subset X-\mathrm{q} \operatorname{coh}_{\mathrm{vff}}$.

(VF4) The full subcategory $X$-qcoh ${ }_{\mathrm{vfl}} \subset X$-qcoh is closed under extensions, infinite direct sums, direct summands, and the passages to the kernels of surjective morphisms of quasi-coherent sheaves.

(VF5) Every quasi-coherent sheaf on $X$ is a quotient sheaf of a very flat quasi-coherent sheaf.

Here we say that a quasi-coherent sheaf $\mathcal{F}$ on $X$ is locally projective if, for every affine open subscheme $U \subset X$, the $\mathcal{O}_{X}(U)$-module $\mathcal{F}(U)$ is projective.

The properties (VF4-VF5) can be summarized by saying that $X$-qcoh $_{\mathrm{vfl}}$ is a resolving subcategory in $X$-qcoh (closedness under direct sums is an extra property not covered by the definition of a resolving subcategory). In particular, it follows that the full subcategory $X-\mathrm{qcoh}{ }_{\mathrm{vfl}}$ inherits an exact category structure from the abelian category $X-\mathrm{qcoh}$.

(VF6) The exact category $X$-qcoh ${ }_{\mathrm{vfl}}$ has finite homological dimension, not exceeding the number of affine open subschemes in an affine open covering of a quasi-compact semi-separated scheme $X$.

(VF7) The embedding of exact categories $X-\mathrm{qcoh}_{\mathrm{vfl}} \longrightarrow X$-qcoh $_{\mathrm{fl}}$ induces an equivalence of their derived categories, $\mathrm{D}\left(X-\mathrm{q} \operatorname{coh}_{\mathrm{vfl}}\right) \simeq \mathrm{D}\left(X-\mathrm{q} \operatorname{coh}_{\mathrm{fl}}\right)$.

The properties (VF1-VF5) hold for the class of all flat quasi-coherent sheaves in place of the class of very flat quasi-coherent sheaves just as well, but the property (VF6) does not. In 
particular, it means that any very flat module ( $=$ very flat quasi-coherent sheaf over an affine scheme) has projective dimension not exceeding 1.

But even flat modules of projective dimension 1 can be complicated. The following property shows the difference between a very flat module and an arbitrary flat module of projective dimension 1. Given a scheme $X$ and a scheme point $x \in X$, let us view $x$ as a scheme (namely, the spectrum of the residue field $k_{x}(X)$ of the point $\left.x \in X\right)$ and denote by $\iota_{x}: x \longrightarrow X$ the natural morphism. For any quasi-coherent sheaf $\mathcal{F}$ on $X$, the $P$-support $\operatorname{PSupp}_{X} \mathcal{F} \subset X$ of $\mathcal{F}$ is the set of all points $x \in X$ such that the quasi-coherent sheaf (or, which is the same in this case, the $k_{x}(X)$-vector space) $\iota_{x}^{*} \mathcal{F}$ does not vanish.

(VF8) For any very flat quasi-coherent sheaf $\mathcal{F}$ on $X$, the subset $\operatorname{PSupp}_{X} \mathcal{F} \subset X$ is open in the Zariski topology of $X$.

Notice that the property (VF8) certainly does not hold for arbitrary flat modules or quasicoherent sheaves, as the example of the flat $\mathbb{Z}$-module $\mathbb{Q}$ (whose P-support consists of the single generic point of $\operatorname{Spec} \mathbb{Z}$ ) already demonstrates.

On the other hand, the properties (VF1-VF4) and (VF8) hold for the class of all locally projective quasi-coherent sheaves in place of the class of all very flat ones. When the analogue of (VF5) holds for locally projective quasi-coherent sheaves, it follows that the analogues of (VF6-VF7) also do.

0.4. Further important properties of the class of very flat quasi-coherent sheaves are related to changing schemes. Let $f: Y \longrightarrow X$ be a morphism of quasi-compact semi-separated schemes, and let $X=\bigcup_{\alpha} U_{\alpha}$ be an open covering of the scheme $X$.

(VF9) The inverse image functor $f^{*}$ takes very flat quasi-coherent sheaves on $X$ to very flat quasi-coherent sheaves on $Y$, that is $f^{*}\left(X-\right.$ qcoh $\left._{\mathrm{vfl}}\right) \subset Y-\mathrm{qcoh}_{\mathrm{vfl}}$.

(VF10) The very flatness property of a quasi-coherent sheaf on $X$ satisfies Zariski descent, that is a quasi-coherent sheaf $\mathcal{F}$ on $X$ is very flat if and only the quasi-coherent sheaf $\left.\mathcal{F}\right|_{U_{\alpha}}$ is very flat on $U_{\alpha}$ for every $\alpha$.

Of course, the classes of flat quasi-coherent sheaves and locally projective quasi-coherent sheaves also satisfy their similar versions of (VF9-VF10).

All of the above having been mentioned, one remaining major property one would like to have for the class of very flat quasi-coherent sheaves is the preservation by direct images with respect to good enough affine morphisms. For example, the class of flat quasi-coherent sheaves is preserved by direct images with respect to all flat affine morphisms $f: Y \longrightarrow X$, where an affine morphism $f$ is said to be flat if the $\mathcal{O}_{X}(U)$-module $\mathcal{O}_{Y}\left(f^{-1}(U)\right)$ is flat for every affine open subscheme $U \subset X$. Equivalently, a morphism $f$ is flat if the local ring $\mathcal{O}_{y}(Y)$ is a flat module over the local ring $\mathcal{O}_{x}(X)$ for every pair of points $x \in X$ and $y \in Y$ such that $f(y)=x$.

What can one say about the preservation of very flatness by the direct images? What are the very flat affine morphisms of schemes? Notice first of all that, according to (VF10), the question reduces to affine schemes, so it suffices to discuss the very flat morphisms of commutative rings. What are these? Before we begin to answer this question, let us define, at long last, the very flat modules.

0.5. Let $R$ be a commutative ring. For every element $r \in R$, we denote by $R\left[r^{-1}\right]$ the result of inverting the element $r$ in $R$, that is, the localization of $R$ with respect to the multiplicative subset $\left\{1, r, r^{2}, r^{3}, \ldots\right\} \subset R$. So $R\left[r^{-1}\right]$ is an $R$-algebra, and in particular, an $R$-module. Using the telescope construction for the inductive limit, one can easily produce a two-term free resolution of the $R$-module $R\left[r^{-1}\right]$, so its projective dimension does not exceed 1 . 
An $R$-module $C$ is said to be $r$-contraadjusted if $\operatorname{Ext}_{R}^{1}\left(R\left[r^{-1}\right], C\right)=0$. An $R$-module $C$ is contraadjusted if it is $r$-contraadjusted for every $r \in R$. An $R$-module $F$ is called very flat if $\operatorname{Ext}_{R}^{1}(F, C)=0$ for every contraadjusted $R$-module $C$.

A somewhat more explicit or more intuitively clear description of the class of all very flat $R$-modules can be given using the notion of a smooth chain of submodules, or a transfinitely iterated extension. Let $F$ be an $R$-module and $\gamma$ be an ordinal. Suppose that for every ordinal $\alpha \leq \gamma$ we are given a submodule $F_{\alpha} \subset F$ such that the following conditions are satisfied:

- $F_{0}=0$ and $F_{\gamma}=F$;

- one has $F_{\alpha} \subset F_{\beta}$ for all $\alpha \leq \beta$;

- and one has $F_{\beta}=\bigcup_{\alpha<\beta} F_{\alpha}$ for all the limit ordinals $\beta \leq \gamma$.

Then, the $R$-module $F$ is said to be a transfinitely iterated extension ("in the sense of the inductive limit") of the $R$-modules $F_{\alpha+1} / F_{\alpha}$, where $0 \leq \alpha<\gamma$.

An $R$-module $F$ is very flat if and only if it is a direct summand of a transfinitely iterated extension of (some $R$-modules isomorphic to) the $R$-modules $R\left[r^{-1}\right]$, where $r \in R$. This is a simple corollary of the main results of the paper [6] applied to the specific situation at hand (see also [22, Corollary 1.1.4]).

The very flat modules over commutative rings were originally defined in the preprint [22, Section 1.1] and further studied in the paper [29]. Why are they relevant to algebraic geometry? One of the simplest answers to this question is this: let $U$ be an affine scheme, and $V \subset U$ be an affine open subscheme. Then $\mathcal{O}(V)$ is a very flat $\mathcal{O}(U)$-module [22, Lemma 1.2.4].

Thus, the class of very flat $R$-modules, defined above in terms of the rings of functions $R\left[r^{-1}\right]$ on the principal affine open subschemes $\operatorname{Spec} R\left[r^{-1}\right] \subset \operatorname{Spec} R, r \in R$, can be equivalently defined in terms of (the underlying $R$-modules of) the rings of functions on arbitrary affine open subschemes in Spec $R$.

A quasi-coherent sheaf $\mathcal{F}$ on a scheme $X$ is said to be very flat if, for every affine open subscheme $U \subset X$, the $\mathcal{O}_{X}(U)$-module $\mathcal{F}(U)$ is very flat.

0.6. Now let us discuss the direct images with respect to affine morphisms. Let $R \longrightarrow S$ be a morphism of commutative rings and $G$ be a flat $S$-module. Assume that $S$ is a flat $R$-module. Then $G$ is a flat $R$-module.

What is the analogue of this assertion for very flat modules? Let $G$ be a very flat $S$-module. What do we need to know about the ring homomorphism $R \longrightarrow S$ in order to conclude that $G$ is a very flat $R$-module? The following definition provides the obvious answer: a commutative ring homomorphism $R \longrightarrow S$ is called very flat if, for every element $s \in S$, the $R$-module $S\left[s^{-1}\right]$ is very flat.

The naïve condition that just the ring $S$ itself should be a very flat $R$-module is not enough. In fact, in Example 9.7 in this paper we demonstrate an example of a commutative ring $S$ with an element $s \in S$ such that $S$ is a free abelian group, but $S\left[s^{-1}\right]$ is not a very flat abelian group or $\mathbb{Z}$-module.

This discussion implies that proving that a particular ring homomorphism is very flat may be not an easy task, even in the most elementary situations. Let $S=R[x]$ be the polynomial ring in one variable over $R$. How does one check that the $R$-module $S\left[s^{-1}\right]$ is very flat for every $s \in S$ ? A geometric proof of this assertion in the case when $R$ contains a field can be found in [22, Theorem 1.7.13]. It is a complicated argument consisting of many steps.

How does one prove that a finite flat morphism is very flat? Let $S=R[x] /(f)$ be the quotient ring of the polynomial ring $R[x]$ by the ideal generated by a unital polynomial $f(x)=x^{n}+f_{n-1} x^{n-1}+\cdots+f_{0}$, where $f_{i} \in R$. So $S$ is a finitely generated free $R$-module 
of rank $n$. How does one check that the $R$-module $S\left[s^{-1}\right]$ is very flat for every $s \in S$ ? Our attempts to look into specific simple instances of this question led to the general theory developed in this paper.

How does one prove that an étale morphism of algebraic varieties is very flat? To all of these questions, we offer one and the same answer: by applying the theorem formulated in the title of the present paper (property (VF15) below, or Main Theorem 1.1 and Corollary 9.1).

0.7. The definition of a very flat morphism of schemes in now in order. A morphism of schemes $f: Y \longrightarrow X$ is called very flat if, for any affine open subschemes $U \subset X$ and $V \subset Y$ such that $f(V) \subset U$, the $\mathcal{O}_{X}(U)$-module $\mathcal{O}_{Y}(V)$ is very flat. An affine morphism $f$ is very flat if and only if, for every affine open subscheme $U \subset X$, the morphism of rings $\mathcal{O}_{X}(U) \longrightarrow \mathcal{O}_{Y}\left(f^{-1}(U)\right)$ is very flat.

(VF11) For any very flat affine morphism $f: Y \longrightarrow X$, the direct image functor $f_{*}$ takes very flat quasi-coherent sheaves on $Y$ to very flat quasi-coherent sheaves on $X$, that is $f_{*}\left(Y-\mathrm{qcoh}_{\mathrm{vfl}}\right) \subset X-\mathrm{qcoh}_{\mathrm{vfl}}$.

(VF12) Let $f: Y \longrightarrow X$ be a morphism of schemes and $X=\bigcup_{\alpha} U_{\alpha}$ be an open covering of the scheme $X$. Then the morphism $f$ is very flat if and only if the morphism $U_{\alpha} \times_{X} Y \longrightarrow U_{\alpha}$ is very flat for every $\alpha$.

(VF13) Let $f: Y \longrightarrow X$ be a morphism of schemes and $Y=\bigcup_{\beta} V_{\beta}$ be an open covering of the scheme $Y$. Then the morphism $f$ is very flat if and only if the morphism $V_{\beta} \longrightarrow X$ is very flat for every $\beta$.

(VF14) Any very flat morphism of schemes $f: Y \longrightarrow X$ is an open map between the underlying topological spaces of $Y$ and $X$.

One can easily check from the definitions that the composition of any two very flat morphisms is very flat. A morphism of rings $R \longrightarrow S$ is said to be universally very flat if the induced morphism of rings $T \longrightarrow S \otimes_{R} T$ is very flat for every ring homomorphism $R \longrightarrow T$. Similarly, a morphism of schemes is said to be universally very flat if it remains very flat after any base change.

(VF15) All flat morphisms of finite presentation between commutative rings are (universally) very flat. All flat morphisms (locally) of finite presentation between schemes are (universally) very flat.

(VF16) Any morphism from a field to a commutative ring is universally very flat. Any morphism from a scheme to the spectrum of a field is universally very flat.

In particular, the property (VF16) means that, for any commutative algebras $S$ and $T$ over a field $k$ and any element $w \in S \otimes_{k} T$, the $T$-module $\left(S \otimes_{k} T\right)\left[w^{-1}\right]$ is very flat. This is the result of our Corollary 9.8 .

To sum up much of the preceding discussion, it follows from the properties (VF11) and (VF15) that direct images with respect to flat affine morphisms of finite presentation take very flat quasi-coherent sheaves to very flat quasi-coherent sheaves. The similar assertion, of course, holds for the flat quasi-coherent sheaves, but the conditions for the direct image to preserve the class of locally projective quasi-coherent sheaves are much more restrictive.

In fact, local projectivity is not even preserved by direct images with respect to affine open embeddings. This is the reason why the proof of property (VF5) for flat or very flat quasi-coherent sheaves does not apply to locally projective ones.

0.8. Let us now provide supporting references for the assertions (VF1-VF14). The properties (VF1-VF2) hold by the definition, and (VF4) is easy to check (see the beginning of [22, 
Section 1.1]). The property (VF3) is [22, Lemma 1.2.1(a)]. The property (VF5) requires a proof; see [22, Lemma 4.1.1].

The property (VF6) is a reformulation of [22, Lemma 4.6.9(a)] (as the contraadjusted very flat quasi-coherent sheaves are the injective objects of the exact category $X-$ qcoh $_{\mathrm{vff}}$, and there are enough of them by [22, Corollary 4.1.4(b)]).

(VF7) is a result of the paper [8]; see [8, Corollary 6.1].

The property (VF8) is [22, Theorem 1.7.6]. The property (VF9) is [22, Lemma 1.2.2(b)], and (VF10) is [22, Lemma 1.2.6(a)]. The property (VF11) is [22, Lemma 1.2.3(b)], (VF12) follows from (VF10), and the property (VF13) is [22, Lemma 1.2.7]. The property (VF14) follows from (VF8) (see [22, Corollary 1.7.8]).

The property (VF15) is the main result of this paper. See Main Theorem 1.1 and Corollary 9.1 .

0.9. Let us say a few more words about (VF14) and (VF15). It is a classical theorem in algebraic geometry that any flat morphism of schemes that is locally of finite presentation is an open map [11, Théorème 2.4.6]. Our results interpret this theorem as the combination of two implications: any flat morphism locally of finite presentation is very flat, and any very flat morphism is open.

Moreover, we have a module version of (VF15) (Main Theorem 1.2), providing a similar interpretation of the assertion in [11] concerning quasi-flat morphisms.

Notice that an arbitrary flat morphism of schemes is, of course, not open: it suffices to consider the morphism $\operatorname{Spec} \mathbb{Q} \longrightarrow \operatorname{Spec} \mathbb{Z}$.

0.10. Before we finish this introduction, let us explain the connection with contraherent cosheaves, which supplied the original motivation for introducing the notion of a very flat module in the preprint [22]. The concept of a contraherent cosheaf on a scheme is dual to that of a quasi-coherent sheaf.

More specifically, a contraherent cosheaf $\mathfrak{P}$ on a scheme $X$ is a rule assigning to every affine open subscheme $U \subset X$ an $\mathcal{O}_{X}(U)$-module $\mathfrak{P}[U]$ and to every pair of embedded affine open subschemes $V \subset U \subset X$ a morphism of $\mathcal{O}_{X}(U)$-modules $\mathfrak{P}[V] \longrightarrow \mathfrak{P}[U]$ such that for every triple of embedded affine open subschemes $W \subset V \subset U \subset X$ the triangle diagram $\mathfrak{P}[W] \longrightarrow \mathfrak{P}[V] \longrightarrow \mathfrak{P}[U]$ is commutative and the following two conditions are satisfied:

(i) for any pair of embedded affine open subschemes $V \subset U \subset X$, the morphism of $\mathcal{O}_{X}(U)$-modules $\mathfrak{P}[V] \longrightarrow \mathfrak{P}[U]$ induces an isomorphism of $\mathcal{O}_{X}(V)$-modules

$$
\operatorname{Hom}_{\mathcal{O}_{X}(U)}\left(\mathcal{O}_{X}(V), \mathfrak{P}[U]\right) \simeq \mathfrak{P}[V]
$$

(ii) for any pair of embedded affine open subschemes $V \subset U \subset X$, one has

$$
\operatorname{Ext}_{\mathcal{O}_{X}(U)}^{1}\left(\mathcal{O}_{X}(V), \mathfrak{P}[U]\right)=0 .
$$

The $\mathcal{O}_{X}(U)$-module $\mathcal{O}_{X}(V)$ is very flat (see Sect. 0.5), so its projective dimension is at most 1 ; that is why the groups $\operatorname{Ext}^{i}$ with $i \geq 2$ are not mentioned in the condition (ii). Moreover, it follows that the condition (ii) is equivalent to the condition that the $\mathfrak{P}[U]$ should be a contraadjusted $\mathcal{O}_{X}(U)$-module. In fact, the category of contraherent cosheaves on an affine scheme $U$ is equivalent to the category of contraadjusted modules over the $\operatorname{ring} \mathcal{O}(U)$.

One can also consider more narrow classes of contraherent cosheaves defined by imposing stricter conditions on the $\mathcal{O}_{X}(U)$-modules $\mathfrak{P}[V]$. An $R$-module $C$ is called (Enochs) cotorsion [7] if $\operatorname{Ext}_{R}^{1}(F, C)=0$ for every flat $R$-module $C$. A contraherent cosheaf $\mathfrak{P}$ is said 
to be locally cotorsion if the $\mathcal{O}_{X}(U)$-module $\mathfrak{P}[U]$ is cotorsion for every affine open subscheme $U \subset X$. A contraherent cosheaf $\mathfrak{J}$ on a scheme $X$ is said to be locally injective if the $\mathcal{O}_{X}(U)$-module $\mathfrak{J}[U]$ is injective for every affine open subscheme $U \subset X[22$, Section 2.2].

0.11. While very flat morphisms of schemes play a role in the theory of quasi-coherent sheaves in that the direct image of a very flat quasi-coherent sheaf with respect to a very flat affine morphism remains very flat, they play an even more important role in the theory of contraherent cosheaves in that the very flatness of a morphism is necessary for the inverse images of contraherent cosheaves to be defined. To be more precise, there are two technical problems associated with the construction of an inverse image of a contraherent cosheaf: the nonexactness of colocalizations and the nonlocality of contraherence. To avoid the second problem, which is not relevant to our present discussion, let us assume that a morphism of schemes $f: Y \longrightarrow X$ is coaffine, that is, for every affine open subscheme $V \subset Y$ there exists an affine open subscheme $U \subset X$ such that $f(V) \subset U$.

In this case, given a contraherent cosheaf $\mathfrak{P}$ on $X$, one defines its inverse image $f ! \mathfrak{P}$ on $Y$ as the rule assigning to an affine open subscheme $V \subset Y$ the $\mathcal{O}_{Y}(V)$-module $\operatorname{Hom}_{\mathcal{O}_{X}(U)}\left(\mathcal{O}_{Y}(V), \mathfrak{P}[U]\right)$. For this definition to work and produce a contraherent cosheaf on $Y$, the morphism $f$ needs to be very flat. More narrow classes of contraherent cosheaves can be pulled back under less restrictive conditions: For example, when $\mathfrak{P}$ is a locally cotorsion contraherent cosheaf on $X$, it suffices that $f$ be a flat morphism of schemes; the inverse image of locally injective contraherent cosheaves can be taken with respect to an arbitrary scheme morphism [22, Section 2.3].

Locally injective contraherent cosheaves form a narrow class dual-analogous to the classes of flat or locally projective quasi-coherent sheaves. The class of locally cotorsion contraherent cosheaves is wider, and one can restrict oneself to these when working over a Noetherian or a locally Noetherian scheme, particularly if such a scheme has finite Krull dimension. (In fact, the locally cotorsion contraherent cosheaves over locally Noetherian schemes are particularly pleasant to work with, owing to the classification of flat cotorsion modules over Noetherian rings [7].) But over a non-Noetherian scheme locally cotorsion contraherent cosheaves are too few, and arbitrary (locally contraadjusted) contraherent cosheaves are needed. Hence, the crucial importance of very flat morphisms of schemes, and consequently of the results of this paper, in the contraherent cosheaf theory.

Finally, the abundance of very flat morphisms of schemes opens the perspective of developing the theory of locally contraadjusted contraherent cosheaves on stacks (e.g., described in terms of flat coverings of finite presentation); cf. [22, Appendix B].

0.12. Some applications of the methods originally developed for the purposes of this paper to the now-classical commutative algebra topic of strongly flat modules [2,3] are worked out in the companion paper [27]. In particular, arbitrary flat modules over a commutative Noetherian ring with countable spectrum are described as direct summands of transfinitely iterated extensions of localizations of the ring at its countable multiplicative subsets, and a related description of the Enochs cotorsion modules is obtained. 


\section{Outline of the arguments}

\subsection{Main theorem}

Let $R$ be a commutative ring. A commutative $R$-algebra $S$ is said to be finitely presented if it can be presented as the quotient algebra $R\left[x_{1}, \ldots, x_{m}\right] / I$ of the algebra of polynomials $R\left[x_{1}, \ldots, x_{m}\right]$ in a finite number of variables with the coefficients in $R$ by a finitely generated ideal $I \subset R\left[x_{1}, \ldots, x_{m}\right]$.

We refer to Sect. 0.5 of the Introduction above and [22, Section 1.1 and C.2] for the definitions related to very flat and contraadjusted $R$-modules (see also [29, Sections 2 and 5]). The following theorem is the main result of this paper. Its particular case for the Noetherian rings (cf. Main Theorem 1.3) was originally proposed as a conjecture in [22, Conjecture 1.7.2].

Main Theorem 1.1 Let $R$ be a commutative ring and $S$ be a finitely presented commutative $R$-algebra. Assume that $S$ is a flat $R$-module. Then $S$ is a very flat $R$-module.

Given a ring $S$, an $S$-module is called finitely presented if it is isomorphic to the cokernel of a morphism between two finitely generated free $S$-modules. The following generalization of Main Theorem 1.1 is useful in some applications.

Main Theorem 1.2 Let $R$ be a commutative ring, $S$ be a finitely presented commutative $R$ algebra, and $F$ be a finitely presented $S$-module. Assume that $F$ is a flat $R$-module. Then $F$ is a very flat $R$-module.

\subsection{Noetherian main lemma}

There are two proofs of Main Theorem 1.2 given in this paper. One of them works for Noetherian rings only. Let us formulate this assertion before explaining the key ideas behind its proof.

Main Theorem 1.3 Let $R$ be a Noetherian commutative ring, $S$ be a finitely generated commutative $R$-algebra, and $F$ be a finitely generated $S$-module. Assume that $F$ is a flat $R$-module. Then $F$ is a very flat $R$-module.

The proof of Main Theorem 1.3 is based on the following main lemma. For any element $r \in R$ and an $R$-module $M$, we denote by $M\left[r^{-1}\right]$ the $R$-module $R\left[r^{-1}\right] \otimes_{R} M$.

Main Lemma 1.4 Let $R$ be a Noetherian commutative ring, $r \in R$ be an element, and $F$ be a flat $R$-module. Then the $R$-module $F$ is very flat if and only if the $R / r R$-module $F / r F$ is very flat and the $R\left[r^{-1}\right]$-module $F\left[r^{-1}\right]$ is very flat.

The "only if" assertion in Main Lemma 1.4 is easy (all extensions of scalars preserve very flatness). The "if" assertion is of key importance.

The argument deducing Main Theorem 1.3 from Main Lemma 1.4 is presented in Sect. 2. It uses Noetherian induction and Grothendieck's generic freeness lemma.

\subsection{Finitely very flat main lemma}

The other proof of Main Theorem 1.2 not only works in full generality, but also provides a stronger result. In order to formulate it, we will need the following definition. 
Let $r_{1}, \ldots, r_{m} \in R$ be a finite collection of elements. We will denote it for brevity by a single letter $\mathbf{r}$. Let us say that an $R$-module $C$ is $\mathbf{r}$-contraadjusted if it is $r_{j}$-contraadjusted for every $j=1, \ldots, m$. An $R$-module $F$ is said to be $\mathbf{r}$-very flat if $\operatorname{Ext}_{R}^{1}(F, C)=0$ for all $\mathbf{r}$-contraadjusted $R$-modules $C$. An $R$-module is $\mathbf{r}$-very flat if and only if it is a direct summand of a transfinitely iterated extension (in the sense of the inductive limit) of $R$ modules isomorphic to $R$ or $R\left[r_{j}^{-1}\right.$ ], $1 \leq j \leq m$ (see [6], [10, Corollary 6.14], or [22, Corollary 1.1.4]).

Let us say that an $R$-module $F$ is finitely very flat if there exists a finite collection of elements $\mathbf{r}=\left\{r_{1}, \ldots, r_{m}\right\}$ in $R$ such that $F$ is $\mathbf{r}$-very flat. To repeat, it means that $F$ is a direct summand of a module admitting a filtration indexed by an arbitrarily large ordinal with all the successive quotients belonging, up to isomorphism, to the finite set of $R$-modules $R$ and $R\left[r_{j}^{-1}\right]$.

Main Theorem 1.5 Let $R$ be a commutative ring, $S$ be a finitely presented commutative $R$ algebra, and $F$ be a finitely presented $S$-module. Assume that $F$ is a flat $R$-module. Then $F$ is a finitely very flat $R$-module.

Actually, there is more to be said about $R$-modules such as in Main Theorem 1.5. Any such $R$-module $F$ can be presented by a countable set of generators with a countable set of relations. Using the Hill Lemma [10, (proof of) Lemma 7.10(H4)], one can deduce that $F$ is a direct summand of an $R$-module admitting a filtration as above indexed by a countable ordinal.

Obviously, Main Theorem 1.5 implies Main Theorem 1.2 (and Main Theorem 1.2 implies Main Theorem 1.1). The proof of Main Theorem 1.5 is based on the next main lemma.

Main Lemma 1.6 Let $R$ be a commutative ring, $r \in R$ be an element, and $F$ be a flat $R$ module. Then the $R$-module $F$ is finitely very flat if and only if the $R / r R$-module $F / r F$ is finitely very flat and the $R\left[r^{-1}\right]$-module $F\left[r^{-1}\right]$ is finitely very flat.

Once again, the "only if" assertion is easy (all extensions of scalars preserve finite very flatness). The "if" assertion is important.

The argument deducing Main Theorem 1.5 from Main Lemma 1.6 is also explained in Sect. 2. It also uses Noetherian induction (even though the rings involved are not Noetherian!). The point is that any finitely presented algebra over a commutative ring can be obtained by an extension of scalars from a finitely presented algebra over a ring finitely generated over the integers.

\subsection{Bounded torsion main lemma}

Notice that Main Lemma 1.4 does not follow from Main Lemma 1.6. One of the reasons why we present two proofs of the main theorem in this paper is because we like the very flat version of the main lemma more than the finitely very flat one.

We do not know whether the Noetherianity condition can be dropped in Main Lemma 1.4, but it can be weakened. Given an element $r$ in a commutative ring $R$ and an $R$-module $M$, we say that the $r$-torsion in $M$ is bounded if there exists an integer $m \geq 1$ such that $r^{n} x=0$ implies $r^{m} x=0$ for all $x \in M$ and $n \geq 1$. The following result is provable with our methods.

Main Lemma 1.7 Let $R$ be a commutative ring and $r \in R$ be an element such that the $r$ torsion in $R$ is bounded. Let $F$ be a flat $R$-module. Then the $R$-module $F$ is very flat if and only if the $R / r R$-module $F / r F$ is very flat and the $R\left[r^{-1}\right]$-module $F\left[r^{-1}\right]$ is very flat. 
The condition on the element $r \in R$ in Main Lemma 1.7 can be relaxed somewhat further. We refer to Remark 5.17 for the discussion.

\subsection{Obtaining contraadjusted modules}

Let us now say a few words about the proofs of the main lemmas. The trick is that these proofs happen on the contraadjusted rather than on the very flat side. One does not really do anything with the given flat module $F$. Instead, one works with an arbitrary contraadjusted $R$-module $C$ (in the case of Main Lemmas 1.4 and 1.7) or with an arbitrary $\mathbf{r}$-contraadjusted $R$-module $C$ (in the case of Main Lemma 1.6).

The contraadjusted modules are described in terms of a certain generation procedure. Given some class of $R$-modules $\mathrm{E} \subset R$-mod, we produce a possibly larger class of $R$ modules $C$ such that whenever one has $\operatorname{Ext}_{R}^{>0}(F, E)=0$ for a given $R$-module $F$ and all $E \in \mathrm{E}$, one also has $\operatorname{Ext}_{R}^{>0}(F, C)=0$ for all $C \in \mathrm{C}$.

In each of the main lemmas, in order to prove that the $R$-module $F$ is (finitely) very flat, one needs to show that $\operatorname{Ext}_{R}^{>0}(F, C)=0$ for every contraadjusted (or $\mathbf{r}$-contraadjusted) $R$-module $C$. After all such $R$-modules $C$ are known to be obtainable using a generation procedure, it remains to check that $\operatorname{Ext}_{R}^{>0}(F, E)=0$ for all the $R$-modules $E$ belonging to the "seed class" $E$. The latter is chosen in such a way that this follows pretty straightforwardly from the conditions imposed on the $R$-module $F$ in the respective main lemma.

The discussion of obtainable modules in this paper occupies two sections. A simpler version of obtainability sufficient for the purposes of Toy Main Lemma 1.8 (see below) is introduced in Sect. 3, and a more sophisticated version required for Main Lemmas 1.4, 1.6, and 1.7 is subsequently defined in Sect. 6 .

\subsection{Toy Main Lemma}

As the idea of the proof described in such terms as above may seem to be too abstract and opaque, we start with a toy example. The proof of the following version of the main lemma is technically much easier; still, it demonstrates many of the same key features that manifest themselves in the proofs of Main Lemmas 1.4, 1.7, and 1.6.

Given a commutative ring $R$ with an element $r \in R$, let us say that an $R$-module $F$ is $r$-very flat if $\operatorname{Ext}_{R}^{1}(F, C)=0$ for every $r$-contraadjusted $R$-module $C$. This is the special case of the above definition of an $\mathbf{r}$-very flat module corresponding to the situation when the finite set of elements $\mathbf{r}$ consists of a single element, $\mathbf{r}=\{r\}$.

Since one has $\operatorname{Ext}_{R}^{1}\left(R, \bigoplus_{x \in X} R\left[r^{-1}\right]\right)=0=\operatorname{Ext}_{R}^{1}\left(R\left[r^{-1}\right], \bigoplus_{x \in X} R\left[r^{-1}\right]\right)$ for any index set $X$, the description of $r$-very flat $R$-modules as the direct summands of transfinitely iterated extensions reduces to the following particularly simple form. An $R$-module $F$ is $r$-very flat if and only if it is a direct summand of an $R$-module $G$ such that there exists a short exact sequence of $R$-modules<smiles>O[Hg]OP[Po]O</smiles>

where $P$ is a free $R$-module and $Q$ is a free $R\left[r^{-1}\right]$-module [10, Corollary 6.13].

Toy Main Lemma 1.8 Let $R$ be a commutative ring, $r \in R$ be an element, and $F$ be a flat $R$-module. Then the $R$-module $F$ is $r$-very flat if and only if the $R / r R$-module $F / r F$ is projective and the $R\left[r^{-1}\right]$-module $F\left[r^{-1}\right]$ is projective.

The proof of Toy Main Lemma 1.8 is given in Sect. 4 . 


\subsection{Contramodules}

Let $R$ be a commutative ring and $r \in R$ be an element. An $R$-module $C$ is said to be an $r$-contramodule [22, Section C.2], [23,24] if $\operatorname{Hom}_{R}\left(R\left[r^{-1}\right], C\right)=0=\operatorname{Ext}_{R}^{1}\left(R\left[r^{-1}\right], C\right)$. This is the key technical concept used in the proofs of all the main lemmas.

In the case when the $r$-torsion in $R$ is bounded, $r$-contramodule $R$-modules are also known under the name of cohomologically I-adically complete modules [18,32], where $I=(r) \subset R$ denotes the principal ideal generated by the element $r \in R$. For an arbitrary element $r$ in a commutative ring $R$, an $R$-module $C$ is said to be $r$-complete if the natural map from $C$ to its $r$-adic completion $C \longrightarrow \lim _{n} C / r^{n} C$ is surjective, and $r$-separated if this map is injective.

All $r$-contramodule $R$-modules (and more generally, all $r$-contraadjusted $R$-modules) are $r$-complete, and all $r$-separated $r$-complete $R$-modules are $r$-contramodules, but the converse implications do not hold. The existence of nonseparated contramodules is an important technical issue which we have to deal with throughout this paper (in particular, in Sects. 4, 5 and 8).

The category of $r$-contramodule $R$-modules is an abelian subcategory in $R$-mod. So is the category of $R\left[r^{-1}\right]$-modules. The idea of the proof of Toy Main Lemma 1.8 is to show that every $r$-contraadjusted $R$-module is obtainable (in the sense discussed in Sect. 1.5) from $r$ contramodule $R$-modules and $R\left[r^{-1}\right]$-modules. Then one shows that all the $r$-contramodule $R$-modules are obtainable from $R / r R$-modules.

The proofs of Main Lemmas 1.4 and 1.7 are more difficult because one has to perform the steps staying inside the class of contraadjusted $R$-modules. So first one shows that every contraadjusted $R$-module is obtainable from a contraadjusted $r$-contramodule $R$-module and a contraadjusted $R\left[r^{-1}\right]$-module, and then, one needs to obtain a contraadjusted $r$ contramodule $R$-module out of contraadjusted $R / r R$-modules. It is the latter step that depends on the Noetherianity/bounded torsion assumptions on $R$.

The homological techniques of working with contramodules that are required for this step were originally developed in [22, Sections C.2 and D.4]. These were originally intended to prepare ground for developing the theory of contraherent cosheaves of contramodules over formal schemes and ind-schemes. We discuss these constructions for $r$-contramodule $R$-modules in a longish Sect. 5. Then we proceed to prove Main Lemmas 1.4 and 1.7 in Sect. 7.

\subsection{Good finite subsets in a ring}

There is a caveat to the discussion of $\mathbf{r}$-contraadjusted $R$-modules in Sect. 1.3, and particularly to the mentioning of "an arbitrary r-contraadjusted $R$-module" in Sect. 1.5, that we should now explain. The $\mathbf{r}$-contraadjusted $R$-module, in the context of the discussion in Sect. 1.5, is arbitrary, but the finite subset $\mathbf{r} \subset R$ is not.

One can say that a finite subset $\mathbf{r} \subset R$ is good if, for every pair of elements $r^{\prime}$ and $r^{\prime \prime} \in \mathbf{r}$, there exists an element $r \in \mathbf{r}$ such that the $R$-algebras $R\left[\left(r^{\prime} r^{\prime \prime}\right)^{-1}\right]$ and $R\left[r^{-1}\right]$ are isomorphic to each other. In this paper, we essentially only work with good finite sets of elements $\mathbf{r}$ in commutative rings $R$.

In more down-to-earth terms, this means that, given a finite set of elements $r_{1}, \ldots, r_{m} \in R$, the first thing we want to do with it is to embed it into a certain larger finite set of elements. For every subset $J \subset\{1, \ldots, m\}$, denote by $r_{J}$ the product $\prod_{j \in J} r_{j}$. The set of elements $\left\{r_{J} \mid J \subset\{1, \ldots, m\}\right\}$ is good. 
In particular, we can now explain how to produce the specific finite set of elements in the ring $R$ appearing in Main Lemma 1.6. Let $\mathbf{s}=\left\{s_{1}, \ldots, s_{p}\right\}$ be a finite set of elements in the quotient ring $R / r R$ such that the $R / r R$-module $F / r F$ is $\mathbf{s}$-very flat, and let $\mathbf{t}=\left\{t_{1}, \ldots, t_{q}\right\}$ be a finite set of elements in the ring of fractions $R\left[r^{-1}\right]$ such that the $R\left[r^{-1}\right]$-module $F\left[r^{-1}\right]$ is t-very flat.

For every $1 \leq i \leq p$, choose a preimage $\tilde{s}_{i} \in R$ of the element $s_{i} \in R / r R$. For every $1 \leq l \leq q$, choose an element $\tilde{t}_{l} \in R$ and an integer $n_{l} \geq 0$ such that $t_{l}=\tilde{t}_{l} / r^{n_{l}}$ in $R\left[r^{-1}\right]$. Consider the collection of all elements $r, \tilde{s}_{1}, \ldots, \tilde{s}_{p}, \tilde{t}_{1}, \ldots, \tilde{t}_{q}$ in $R$, and form a good finite subset $\mathbf{r} \subset R$ containing all these elements.

Then the $R$-module $F$ is $\mathbf{r}$-very flat. This is what (the proof of) Main Lemma 1.6 actually tells.

\section{$1.9 r^{\times}$-very flat theorem}

In order to prove Main Lemma 1.6, we reformulate it in the following form, which is a kind of multi-element version of Toy Main Lemma 1.8.

We will use the following notation. Let $r_{1}, \ldots, r_{m}$ be a finite set of elements in a commutative ring $R$. As above, for every subset $J \subset\{1, \ldots, m\}$ we put $r_{J}=\prod_{j \in J} r_{j}$. Furthermore, put $K=\{1, \ldots, m\} \backslash J$ and denote by $R_{J}=\left(R / \sum_{k \in K} r_{k} R\right)\left[r_{J}^{-1}\right]$ the ring obtained by annihilating all the elements $r_{k}, k \in K$, and inverting all the elements $r_{j}, j \in J$ in the ring $R$. Let us denote the finite set of elements $\left\{r_{J} \mid J \subset\{1, \ldots, m\}\right\}$ in the ring $R$ by $\mathbf{r}^{\times}$.

Theorem 1.9 Let $R$ be a commutative ring, $r_{1}, \ldots, r_{m} \in R$ be a finite set of its elements, and $F$ be a flat $R$-module. Then the $R$-module $F$ is $\mathbf{r}^{\times}$-very flat if and only if the $R_{J}$-module $R_{J} \otimes_{R} F$ is projective for every subset $J \subset\{1, \ldots, m\}$.

Deducing Main Lemma 1.6 from Theorem 1.9 is easy, but the proof of Theorem 1.9 is quite involved. We present both these arguments in Sect. 8.

\subsection{Flat lemma}

Looking into the argument outlined in Sect. 1.5 suggests that the assumptions in the main lemmas can be weakened. Indeed, given that we assume both the $R / r R$-module $F / r F$ and the $R\left[r^{-1}\right]$-module $F\left[r^{-1}\right]$ to be, at least, very flat, why do we need to assume the $R$-module $F$ to be flat, on top of that?

In fact, the condition that the $R$-module $F$ is flat can be replaced in these assertions by the condition of vanishing of certain specific Tor modules related to the element $r$. The following lemma provides more information, however.

Lemma 1.10 Let $R$ be a commutative ring and $r \in R$ be an element. Let $F$ be an $R$-module such that $\operatorname{Tor}_{1}^{R}(R / r R, F)=0=\operatorname{Tor}_{2}^{R}(R / r R, F)$. Then the $R$-module $F$ is flat if and only if the $R / r R$-module $F / r F$ is flat and the $R\left[r^{-1}\right]$-module $F\left[r^{-1}\right]$ is flat.

Lemma 1.10 is even easier to prove than Toy Main Lemma 1.8, as there are no contramodules in the proof of Lemma 1.10. Instead, $r$-torsion modules are used. We prove both these "toy" lemmas in Sect. 4. 


\subsection{Surjective descent}

Notice that for any element $r$ in a commutative ring $R$ the ring $R / r R \oplus R\left[r^{-1}\right]$ is a finitely presented commutative $R$-algebra. Furthermore, the ring homomorphism $R \longrightarrow R / r R \oplus$ $R\left[r^{-1}\right]$ induces a bijective map (but, of course, not a homeomorphism) of the spectra

$$
\operatorname{Spec} R / r R \sqcup \operatorname{Spec} R\left[r^{-1}\right]=\operatorname{Spec}\left(R / r R \oplus R\left[r^{-1}\right]\right) \longrightarrow \operatorname{Spec} R \text {. }
$$

The following two results generalize Main Lemmas 1.4 and 1.6, respectively.

Theorem 1.11 Let $R$ be a Noetherian commutative ring and $S$ be a finitely generated commutative $R$-algebra such that the induced map of the spectra $\operatorname{Spec} S \longrightarrow \operatorname{Spec} R$ is surjective. Let $F$ be a flat $R$-module. Then the $R$-module $F$ is very flat if and only if the $S$-module $S \otimes_{R} F$ is very flat.

Theorem 1.12 Let $R$ be a commutative ring and $S$ be a finitely presented commutative $R$ algebra such that the induced map of the spectra $\operatorname{Spec} S \longrightarrow \operatorname{Spec} R$ is surjective. Let $F$ be a flat $R$-module. Then the $R$-module $F$ is finitely very flat if and only if the $S$-module $S \otimes_{R} F$ is finitely very flat.

The proofs of Theorems 1.11 and 1.12 are based on the main lemmas which they generalize. Theorem 1.11 is deduced from Main Lemma 1.4, and Theorem 1.12 is deduced from Main Lemma 1.6. This is done in Sect. 10.

The arguments use Noetherian induction very similar to the one that we use in order to deduce the main theorems from the main lemmas, and the same generic freeness lemma (cf. the discussion in Sects. 1.2-1.3).

\section{Main lemma implies main theorem}

The proofs of the main theorems are based on the following generic freeness result.

Lemma 2.1 Let $R$ be a Noetherian commutative integral domain, $S$ be a finitely generated commutative $R$-algebra, and $F$ be a finitely generated $S$-module. Then there exists a nonzero element $a \in R$ such that the $R\left[a^{-1}\right]$-module $F\left[a^{-1}\right]$ is free.

Proof This is [11, Lemme 6.9.2]. For a generalization, see [12, Theorem 24.1].

For ease of reference, let us also formulate here the assertion about preservation of (finite) very flatness by extensions of scalars.

Lemma 2.2 (a) Let $R \longrightarrow R^{\prime}$ be a morphism of commutative rings and $F$ be a very flat $R$-module. Then the $R^{\prime}$-module $R^{\prime} \otimes_{R} F$ is also very flat.

(b) Let $R \longrightarrow R^{\prime}$ be a morphism of commutative rings and $F$ be a finitely very flat $R$ module. Then the $R^{\prime}$-module $R^{\prime} \otimes_{R} F$ is also finitely very flat.

Proof Part (a) is [22, Lemma 1.2.2(b)], and the proof of part (b) is similar. If $\mathbf{r}=\left\{r_{1}, \ldots, r_{m}\right\}$ is a finite set of elements in $R$ such that the $R$-module $F$ is $\mathbf{r}$-very flat, then the $R^{\prime}$-module $R^{\prime} \otimes_{R} F$ is $\mathbf{r}^{\prime}$-very flat, where $\mathbf{r}^{\prime}=\left\{r_{1}^{\prime}, \ldots, r_{m}^{\prime}\right\}$ and $r_{j}^{\prime} \in R^{\prime}$ denotes the image of the element $r_{j} \in R$ under the ring homomorphism $R \longrightarrow R^{\prime}$.

The proofs in this section are also based on the classical technique of Noetherian induction, which can be formulated as follows. 
Noetherian Induction Principle Let $R$ be a Noetherian commutative ring. Then there cannot exist a sequence of rings $R_{n}, n \geq 0$, and nonzero elements $r_{n} \in R_{n}$ such that $R_{0}=R$ and $R_{n+1}=R_{n} / r_{n} R_{n}$ for all $n \geq 0$.

Proof Denote by $\tilde{r}_{n} \in R$ some preimages of the elements $r_{n} \in R_{n}$, and let $I_{n}$ be the ideal in $R$ generated by the elements $\tilde{r}_{0}, \ldots, \tilde{r}_{n-1}$. Then we have $R / I_{n} \simeq R_{n}$, and the ideals $I_{n} \subset R$ form an infinite ascending chain $0 \varsubsetneqq I_{0} \varsubsetneqq I_{1} \varsubsetneqq I_{2} \varsubsetneqq \cdots \subset R$, contradicting the assumption of Noetherianity of the ring $R$.

We start with explaining the proof of Main Theorem 1.3, which highlights the key ideas of the argument. Then we proceed to prove the stronger Main Theorem 1.5.

The following proposition is a particular case of Main Theorem 1.3 from which the general case is deduced.

Proposition 2.3 Let $R$ be a Noetherian commutative ring, $S$ be a finitely generated commutative $R$-algebra, and $F$ be a finitely generated $S$-module. Assume that $F$ is a flat $R$-module, and that for every nonzero element $r \in R$ the $R / r R$-module $F / r F$ is very flat. Then $F$ is a very flat $R$-module.

Proof Case I: suppose that the ring $R$ has zero-divisors, that is, there exists a pair of nonzero elements $a, b \in R$ such that $a b=0$ in $R$. By assumption, the $R / a R$-module $F / a F$ is very flat and the $R / b R$-module $F / b F$ is very flat. Now the localization morphism $R \longrightarrow R\left[a^{-1}\right]$ factorizes as $R \longrightarrow R / b R \longrightarrow R\left[a^{-1}\right]$, and hence, very flatness of the $R / b R$-module $F / b F$ implies very flatness of the $R\left[a^{-1}\right]$-module $F\left[a^{-1}\right]$ by Lemma 2.2(a). Both the $R / a R$ module $F / a F$ and the $R\left[a^{-1}\right]$-module $F\left[a^{-1}\right]$ being very flat, very flatness of the $R$-module $F$ follows by Main Lemma 1.4.

Case II: now suppose that the ring $R$ is an integral domain. By Lemma 2.1, there exists a nonzero element $a \in R$ such that the $R\left[a^{-1}\right]$-module $F\left[a^{-1}\right]$ is free. By assumption, the $R / a R$-module $F / a F$ is very flat. Once again, very flatness of the $R$-module $F$ follows by virtue of Main Lemma 1.4.

Proof of Main Theorem 1.3 Assume that the $R$-module $F$ is not very flat. By Proposition 2.3, it then follows that there exists a nonzero element $r \in R$ such that the $R / r R$-module $F / r F$ is not very flat.

Now $R / r R$ is a Noetherian ring, $S / r S$ is a finitely generated $R / r R$-algebra, and $F / r F$ is a finitely generated $S / r S$-module. Also, $F / r F$ is a flat $R / r R$-module. Set $R_{1}=R / r R$, $S_{1}=S / r S$, and $F_{1}=F / r F$. Applying Proposition 2.3 again, we find a nonzero element $r_{1} \in R_{1}$ such that the $R_{1} / r_{1} R_{1}$-module $F_{1} / r_{1} F_{1}$ is not very flat.

Proceeding in this fashion, we produce an infinite sequence of nonzero elements $r_{n} \in R_{n}$ and quotient rings $R_{n+1}=R_{n} / r_{n} R_{n}$, starting from $R_{0}=R$ and $r_{0}=r$. According to the Noetherian induction principle, this is impossible. The contradiction proves that the $R$ module $F$ is very flat.

The proof of Main Theorem 1.5 is based on the following lemma, which allows to apply Noetherian induction to some problems involving non-Noetherian rings.

Lemma 2.4 Let $R$ be a commutative ring, $S$ be a finitely presented commutative $R$-algebra, and $F$ be a finitely presented $S$-module. Then there exist a commutative ring $\bar{R}$ finitely generated over the ring of integers $\mathbb{Z}$, a ring homomorphism $\bar{R} \longrightarrow R$, a finitely generated commutative $\bar{R}$-algebra $\bar{S}$, and a finitely generated $\bar{S}$-module $\bar{F}$ such that $S=R \otimes_{\bar{R}} \bar{S}$ and $F=S \otimes_{\bar{S}} \bar{F}=R \otimes_{\bar{R}} \bar{F}$. 
Proof Let $I \subset R\left[x_{1}, \ldots, x_{m}\right]$ be a finitely generated ideal such that the $R$-algebra $R\left[x_{1}, \ldots, x_{m}\right] / I$ is isomorphic to $S$, and let $N \subset S^{n}$ be a finitely generated submodule such that the $S$-module $S^{n} / N$ is isomorphic to $F$. Let $f_{1}\left(x_{1}, \ldots, x_{m}\right), \ldots, f_{k}\left(x_{1}, \ldots, x_{m}\right) \in$ $R\left[x_{1}, \ldots, x_{m}\right]$ be a finite set of generators of the ideal $I$, and let $\left(v_{1,1}, \ldots, v_{1, n}\right), \ldots$, $\left(v_{l, 1}, \ldots, v_{l, n}\right) \in S^{n}$ be a finite set of generators of the $S$-module $N$. Finally, let $q_{i, j} \in$ $R\left[x_{1}, \ldots, x_{m}\right]$ be some preimages of the elements $v_{i, j} \in S$.

Let $\left\{r_{\alpha}\right\}$ denote the set of all coefficients of the polynomials $f_{h}\left(x_{1}, \ldots, x_{m}\right), 1 \leq h \leq k$, and $q_{i, j}\left(x_{1}, \ldots, x_{m}\right), 1 \leq i \leq l, 1 \leq j \leq n$. So $\left\{r_{\alpha}\right\}$ is a finite set of elements of the ring $R$. Set $\bar{R}$ to be the subring in $R$ generated by the elements $\left\{r_{\alpha}\right\}$. Then the polynomials $f_{h}$ and $q_{i j}$ belong to the subring $\bar{R}\left[x_{1}, \ldots, x_{m}\right] \subset R\left[x_{1}, \ldots, x_{m}\right]$.

Now we can set $\bar{I}$ to be the ideal in $\bar{R}\left[x_{1}, \ldots, x_{m}\right]$ generated by the polynomials $f_{1}, \ldots$, $f_{k}$, and put $\bar{S}=\bar{R}\left[x_{1}, \ldots, x_{m}\right] / \bar{I}$. Furthermore, let $\bar{v}_{i j} \in \bar{S}$ denote the images of the elements $q_{i j} \in \bar{R}\left[x_{1}, \ldots, x_{m}\right]$. Set $\bar{N}$ to be the submodule in $\bar{S}^{n}$ generated by the vectors $\left(\bar{v}_{1,1}, \ldots, \bar{v}_{1, n}\right), \ldots,\left(\bar{v}_{l, 1}, \ldots, \bar{v}_{l, n}\right)$, and put $\bar{F}=\bar{S}^{n} / \bar{N}$.

Alternatively, one could set $R$ to be the polynomial ring $\mathbb{Z}\left[z_{\alpha}\right]$ in the free variables $z_{\alpha}$ corresponding bijectively to the elements $r_{\alpha} \in R$. Then one would have a commutative ring homomorphism $\bar{R} \longrightarrow R$ taking $z_{\alpha}$ to $r_{\alpha}$. It would remain to lift the polynomials $f_{h}$ and $q_{i j} \in R\left[x_{1}, \ldots, x_{m}\right]$ to some polynomials $\bar{f}_{h}$ and $\bar{q}_{i j} \in \bar{R}\left[x_{1}, \ldots, x_{m}\right]$, and proceed with the constructions of an $\bar{R}$-algebra $\bar{S}$ and an $\bar{S}$-module $\bar{F}$ in the way similar to the above.

Remark 2.5 Notice that, when the $R$-module $F$ is flat, there is no claim in Lemma 2.4 that the $\bar{R}$-module $\bar{F}$ is flat.

Similarly to the above, we now proceed to formulate a particular case of Main Theorem 1.5 from which the general case will be deduced.

Proposition 2.6 Let $\bar{R}$ be a Noetherian commutative ring, $\bar{S}$ be a finitely generated commutative $\bar{R}$-algebra, and $\bar{F}$ be a finitely generated $\bar{S}$-module. Let $R$ be a commutative $\bar{R}$-algebra. Assume that $F=R \otimes \bar{R} \bar{F}$ is a flat $R$-module, and that for every nonzero element $r \in \bar{R}$ the $R / r R$-module $F / r F$ is finitely very flat. Then $F$ is a finitely very flat $R$-module.

Proof Case I: suppose that the $\operatorname{ring} \bar{R}$ has zero-divisors, that is, there exists a pair of elements $a \neq 0 \neq b$ in $\bar{R}$ such that $a b=0$ in $\bar{R}$. By assumption, the $R / a R$-module $F / a F$ is finitely very flat and the $R / b R$-module $F / b F$ is finitely very flat. Arguing as in the proof of Proposition 2.3 and using Lemma 2.2(b), we see that the $R\left[a^{-1}\right]$-module $F\left[a^{-1}\right]$ is finitely very flat. Both the $R / a R$-module $F / a F$ and the $R\left[a^{-1}\right]$-module $F\left[a^{-1}\right]$ being finitely very flat, finite very flatness of the $R$-module $F$ follows by Main Lemma 1.6.

Case II: now suppose that the ring $\bar{R}$ is an integral domain. By Lemma 2.1, there exists a nonzero element $a \in \bar{R}$ such that the $\bar{R}\left[a^{-1}\right]$-module $\bar{F}\left[a^{-1}\right]$ is free. It follows that the $R\left[a^{-1}\right]$-module $F\left[a^{-1}\right] \simeq R \otimes_{R} \bar{F}\left[a^{-1}\right]$ is free as well. By assumption, the $R / a R$-module $F / a F$ is finitely very flat. As above, finite very flatness of the $R$-module $F$ follows by means of Main Lemma 1.6.

Proof of Main Theorem 1.5 Starting from a commutative ring $R$, a finitely presented commutative $R$-algebra $S$, and a finitely presented $S$-module $F$, we first of all apply Lemma 2.4. This produces a Noetherian commutative ring $\bar{R}$ with a ring homomorphism $\bar{R} \longrightarrow R$, a finitely generated commutative $\bar{R}$-algebra $\bar{S}$, and a finitely generated $\bar{S}$-module $\bar{F}$ such that $F \simeq R \otimes_{R} \bar{F}$.

By the assumptions of the theorem, the $R$-module $F$ is flat. Assume that it is not finitely very flat. By Proposition 2.6, it then follows that there exists a nonzero element $r \in \bar{R}$ such that the $R / r R$-module $F / r F$ is not finitely very flat. 
Now $\bar{R} / r \bar{R}$ is a Noetherian ring, $\bar{S} / r \bar{S}$ is a finitely generated $\bar{R} / r \bar{R}$-algebra, and $\bar{F} / r \bar{F}$ is a finitely generated $\bar{S} / r \bar{S}$-module. Furthermore, $R / r R$ is an $\bar{R} / r \bar{R}$-algebra and $F / r F \simeq$ $R / r R \otimes_{\bar{R} / r \bar{R}} \bar{F} / r \bar{F}$ is a flat $R / r R$-module. Set $\bar{R}_{1}=\bar{R} / r \bar{R}, \bar{S}_{1}=\bar{S} / r \bar{S}, \bar{F}_{1}=\bar{F} / r \bar{F}$, $R_{1}=R / r R$, and $F_{1}=F / r F$. Applying Proposition 2.6, we find a nonzero element $r_{1} \in \bar{R}_{1}$ such that the $R_{1} / r R_{1}$-module $F_{1} / r F_{1}$ is not very flat.

Proceeding in this way, we produce an infinite sequence of nonzero elements $r_{n} \in \bar{R}_{n}$ and quotient rings $\bar{R}_{n+1}=\bar{R}_{n} / r_{n} \bar{R}_{n}$, starting from $\bar{R}_{0}=\bar{R}$ and $r_{0}=r$. The argument by contradiction with Noetherian induction principle (applied to the Noetherian ring $\bar{R}$ ) finishes in the same way as in the above proof of Main Theorem 1.3.

\section{Obtainable modules I}

In this section, we discuss the "simple obtainability." The discussion of the more complicated "two-sorted obtainability" is postponed to Sect. 6.

In this section, we work over an associative (not necessarily commutative) ring $R$. We denote by $R$-mod the abelian category of left $R$-modules and by $\bmod -R$ the abelian category of right $R$-modules.

Let $\mathrm{E}$ and $\mathrm{F} \subset R$-mod be two classes of left $R$-modules. Denote by ${ }_{\geq \geq 1} \mathrm{E} \subset R$-mod the class of all left $R$-modules $F$ such that $\operatorname{Ext}_{R}^{i}(F, E)=0$ for all $E \in \mathrm{E}$ and $i \geq 1$, and by $\mathrm{F}^{\perp \geq 1} \subset R$-mod the class of all left $R$-modules $C$ such that $\operatorname{Ext}_{R}^{i}(F, C)=0$ for all $F \in \mathrm{F}$ and $i \geq 1$.

Let $\mathrm{E} \subset R$-mod be a fixed class of left $R$-modules. Set $\mathrm{F}={ }^{\perp_{\geq 1}} \mathrm{E}$ and $\mathrm{C}=\mathrm{F}^{\perp_{\geq 1}}$. Our aim is to describe (to the extent possible, which may depend on the situation at hand) the class $C$ in terms of the class $E$.

The following definition of a transfinitely iterated extension is dual to that from Sect. 0.5. Let $C$ be a left $R$-module and $\delta$ be an ordinal. Suppose that for every ordinal $\alpha \leq \delta$ we are given a left $R$-module $C_{\alpha}$ and for every pair of ordinals $\alpha<\beta \leq \delta$ we are given an $R$-module morphism $C_{\beta} \longrightarrow C_{\alpha}$ such that the following conditions are satisfied:

- $C_{0}=0$ and $C_{\delta}=C$;

- the triangle diagrams $C_{\gamma} \longrightarrow C_{\beta} \longrightarrow C_{\alpha}$ are commutative for all triples of ordinals $\alpha<\beta<\gamma \leq \delta$

- the induced morphism into the projective limit $C_{\beta} \longrightarrow \lim _{\alpha<\beta} C_{\alpha}$ is an isomorphism for all limit ordinals $\beta \leq \delta$;

- the morphism $C_{\alpha+1} \longrightarrow C_{\alpha}$ is surjective for all ordinals $\alpha<\delta$.

Let $D_{\alpha}$ denote the kernel of the morphism $C_{\alpha+1} \longrightarrow C_{\alpha}$. Then the $R$-module $C$ is said to be a transfinitely iterated extension (in the sense of the projective limit) of the $R$-modules $D_{\alpha}$, where $0 \leq \alpha<\delta$.

Lemma 3.1 Let $F$ and $C$ be left $R$-modules such that $C$ is a transfinitely iterated extension (in the sense of the projective limit) of left $R$-modules $D_{\alpha}$. Assume that $\operatorname{Ext}_{R}^{1}\left(F, D_{\alpha}\right)=0$ for all $\alpha$. Then $\operatorname{Ext}_{R}^{1}(F, C)=0$.

Proof This is the dual version of the Eklof Lemma [6, Lemma 1]. See [6, Proposition 18] (or, for a generalization to arbitrary abelian categories, [26, Lemma 4.5]).

Lemma 3.2 For any class of left $R$-modules $F \subset R$-mod, the class of left $R$-modules $C=$ $F^{\perp \geq 1}$ is closed under the passages to direct summands, extensions, cokernels of injective 
morphisms, infinite products, and transfinitely iterated extensions in the sense of the projective limit.

Proof Notice that, for any class of left $R$-modules $\mathrm{G} \subset R$-mod, the class $\mathrm{G}^{\perp_{1}} \subset R$-mod consisting of all the left $R$-modules $C$ such that $\operatorname{Ext}_{R}^{1}(G, C)=0$ for all $G \in \mathrm{G}$ is closed under all the mentioned operations with the possible exception of the cokernels of injective morphisms. This follows from Lemma 3.1 (notice also that extensions and infinite products are particular cases of the transfinitely iterated extensions in the sense of the projective limit). Now let $G$ be the closure of $F$ with respect to the operation of the passage to a syzygy module. Then $F^{\perp_{\geq 1}}=G^{\perp_{1}}$. Checking that $F^{\perp_{\geq 1}}$ is closed under the cokernels of injective morphisms is easy.

The following definition plays a key role.

Definition 3.3 The class of all left $R$-modules simply right obtainable from a given class $\mathrm{E} \subset R$-mod is defined as the (obviously, unique) minimal class of left $R$-modules containing $E$ and closed under the operations of the passage to a direct summand, an extension, the cokernel of an injective morphism, an infinite product, or a transfinitely iterated extension in the sense of the projective limit.

Lemma 3.4 For any class of left $R$-modules $\mathrm{E} \subset R$-mod, all the left $R$-modules simply right obtainable from $\mathrm{E}$ belong to the class $\mathrm{C}=\left({ }^{{ } \geq 1} \mathrm{E}\right)^{\perp_{\geq 1}} \subset R$-mod.

Proof Follows from Lemma 3.2.

Now let us formulate the dual definition. For any class of left $R$-modules $\mathrm{M}$, we denote by $\mathrm{T}_{\geq 1} \mathrm{M} \subset \bmod -R$ the class of all right $R$-modules $N$ such that $\operatorname{Tor}_{i}^{R}(N, M)=0$ for all $M \in \mathrm{M}$ and $i \geq 1$. Similarly, for any class of right $R$-modules $\mathrm{N}$, we denote by $\mathrm{N}^{\top} \geq 1 \subset R$-mod the class of all left $R$-modules $M$ such that $\operatorname{Tor}_{i}^{R}(N, M)=0$ for all $N \in \mathrm{N}$ and $i \geq 1$.

Definition 3.5 The class of all left $R$-modules simply left obtainable from a given class $\mathrm{M} \subset R$-mod is defined as the (obviously, unique) minimal class of left $R$-modules containing $M$ and closed under the operations of the passage to a direct summand, an extension, the kernel of a surjective morphism, an infinite direct sum, or a transfinitely iterated extension in the sense of the inductive limit.

Lemma 3.6 (a) For any class of left $R$-modules $\mathrm{M} \subset R$-mod, all the left $R$-modules simply left obtainable from $\mathrm{M}$ belong to the class ${ }^{\perp \geq 1}\left(\mathrm{M}^{\perp_{\geq 1}}\right) \subset R$-mod.

(b) For any class of left $R$-modules $M \subset R$-mod, all the left $R$-modules simply left obtainable from $M$ belong to the class $\left({ }^{\top} \geq 1 \mathrm{M}\right)^{\top} \geq 1 \subset R$-mod.

Proof Part (a) is provable in the same way as Lemma 3.4 (using the classical Eklof Lemma [6, Lemma 1] in place of its dual version which we formulated as Lemma 3.1). Part (b) can be deduced from part (a) using the functor $\operatorname{Hom}_{\mathbb{Z}}(-, \mathbb{Q} / \mathbb{Z})$, or proved directly using the fact that the functor Tor preserves filtered inductive limits.

Remark 3.7 In the context of the arguments in this paper (see the discussion in Sect. 1.5), we will not nearly use the full strength of Definitions 3.3 and 3.5. In particular, the passage to direct summands will not be used, and we will only use countable products/sums, and the transfinitely iterated extensions indexed by the ordinal $\omega=\mathbb{Z}_{\geq 0}$ of nonnegative integers only. We will call the latter "infinitely iterated extensions." 


\section{Toy examples of a main lemma}

Part (a) of the following lemma will be used in the proofs of all the main lemmas.

Lemma 4.1 Let $R \longrightarrow R^{\prime}$ be a morphism of commutative rings and $F$ be an $R$-module. Assume that $\operatorname{Tor}_{i}^{R}\left(R^{\prime}, F\right)=0$ for all $i \geq 1$. Then

(a) for any $R^{\prime}$-module $C$ and all $i \geq 0$ there is a natural isomorphism $\operatorname{Ext}_{R}^{i}(F, C) \simeq$ $\operatorname{Ext}_{R^{\prime}}^{i}\left(R^{\prime} \otimes_{R} F, C\right)$;

(b) for any $R^{\prime}$-module $N$ and all $i \geq 0$ there is a natural isomorphism $\operatorname{Tor}_{i}^{R}(N, F) \simeq$ $\operatorname{Tor}_{i}^{R^{\prime}}\left(N, R^{\prime} \otimes_{R} F\right)$.

Proof It suffices to notice that, in the assumptions of the lemma, for any projective resolution $P$. $\longrightarrow F$ of the $R$-module $F$, the complex $R^{\prime} \otimes_{R} P$. is a projective resolution of the $R^{\prime}$-module $R^{\prime} \otimes_{R} F$.

Part (b) of the following generalization of Lemma 4.1 will be used in the proof of Lemma 1.10 in this section.

Lemma 4.2 Let $R \longrightarrow R^{\prime}$ be a morphism of commutative rings, $F$ be an $R$-module, and $n \geq 1$ be an integer. Assume that $\operatorname{Tor}_{i}^{R}\left(R^{\prime}, F\right)=0$ for all $1 \leq i \leq n$. Then

(a) for any $R^{\prime}$-module $C$ and all $0 \leq i \leq n$ there is a natural isomorphism $\operatorname{Ext}_{R}^{i}(F, C) \simeq$ $\operatorname{Ext}_{R^{\prime}}^{i}\left(R^{\prime} \otimes_{R} F, C\right)$;

(b) for any $R^{\prime}$-module $N$ and all $0 \leq i \leq n$ there is a natural isomorphism $\operatorname{Tor}_{i}^{R}(N, F) \simeq$ $\operatorname{Tor}_{i}^{R^{\prime}}\left(N, R^{\prime} \otimes_{R} F\right)$.

Proof Similarly to the previous proof, let $P_{n+1} \longrightarrow P_{n} \longrightarrow \cdots \longrightarrow P_{1} \longrightarrow P_{0}$ be an initial fragment of a projective resolution of the $R$-module $F$. Then $R^{\prime} \otimes_{R} P_{n+1} \longrightarrow R^{\prime} \otimes_{R} P_{n} \longrightarrow$ $\cdots \longrightarrow R^{\prime} \otimes_{R} P_{1} \longrightarrow R^{\prime} \otimes_{R} P_{0}$ is an initial fragment of a projective resolution of the $R^{\prime}-$ module $R^{\prime} \otimes_{R} F$.

We start with proving Lemma 1.10 before proceeding to prove the slightly more complicated Toy Main Lemma 1.8.

Let $R$ be a commutative ring and $r \in R$ be an element. An $R$-module $M$ is said to be $r$-torsion if for every $x \in M$ there exists $n \geq 1$ such that $r^{n} x=0$ in $M$. We denote the full subcategory of $r$-torsion submodules by $R$ - $\bmod _{r \text {-tors }} \subset R$-mod.

For any $R$-module $M$ and an element $t \in R$, denote by ${ }_{t} M \subset M$ the submodule formed by all the elements $x \in M$ such that $t x=0$ in $M$. Furthermore, denote by $\Gamma_{r}(M) \subset M$ the maximal $r$-torsion submodule in $M$; so $\Gamma_{r}(M)=\bigcup_{n \geq 1} r^{n} M$. The functor $\Gamma_{r}: R-\bmod \longrightarrow$ $R$ - $\bmod _{r \text {-tors }}$ is right adjoint to the embedding functor $R$ - $\bmod _{r \text {-tors }} \longrightarrow R$-mod.

Lemma 4.3 Any $r$-torsion $R$-module can be obtained as an infinitely iterated extension, in the sense of the inductive limit, of $R / r R$-modules (viewed as $R$-modules via the restriction of scalars).

Proof Let $M$ be an $r$-torsion $R$-module. Then we have $M=\bigcup_{n \geq 0} r^{n} M$, and the successive quotients ${ }_{r^{n+1}} M / r^{n} M$ are $R / r R$-modules.

Proof of Lemma 1.10 Let $N$ be an $R$-module. We have to show that $\operatorname{Tor}_{1}^{R}(N, F)=0$. 
Denote by $l_{N}$ the $R$-module morphism $N \longrightarrow N\left[r^{-1}\right]$. There are two short exact sequences of $R$-modules

$$
\begin{aligned}
& 0 \longrightarrow \operatorname{ker}\left(l_{N}\right) \longrightarrow N \longrightarrow \operatorname{im}\left(l_{N}\right) \longrightarrow 0, \\
& 0 \longrightarrow \operatorname{im}\left(l_{N}\right) \longrightarrow N\left[r^{-1}\right] \longrightarrow \operatorname{coker}\left(l_{N}\right) \longrightarrow 0,
\end{aligned}
$$

where $\operatorname{ker}\left(l_{N}\right)=\Gamma_{r}(N)$ and $\operatorname{coker}\left(l_{N}\right)=\operatorname{coker}\left(l_{R}\right) \otimes_{R} N$. From the related long exact sequences of $\operatorname{Tor}_{*}^{R}(-, F)$

$$
\begin{aligned}
& \cdots \longrightarrow \operatorname{Tor}_{1}^{R}\left(\operatorname{ker}\left(l_{N}\right), F\right) \longrightarrow \operatorname{Tor}_{1}^{R}(N, F) \longrightarrow \operatorname{Tor}_{1}^{R}\left(\operatorname{im}\left(l_{N}\right), F\right) \longrightarrow \cdots, \\
& \cdots \longrightarrow \operatorname{Tor}_{2}^{R}\left(\operatorname{coker}\left(l_{N}\right), F\right) \longrightarrow \operatorname{Tor}_{1}^{R}\left(\operatorname{im}\left(l_{N}\right), F\right) \longrightarrow \operatorname{Tor}_{1}^{R}\left(N\left[r^{-1}\right], F\right) \longrightarrow \cdots
\end{aligned}
$$

we see that, in order to prove that $\operatorname{Tor}_{1}^{R}(N, F)=0$, it suffices to check that $\operatorname{Tor}_{1}^{R}\left(\operatorname{ker}\left(l_{N}\right), F\right)=$ $0, \operatorname{Tor}_{1}^{R}\left(N\left[r^{-1}\right], F\right)=0$, and $\operatorname{Tor}_{2}^{R}\left(\operatorname{coker}\left(l_{N}\right), F\right)=0$.

Now $N\left[r^{-1}\right]$ is an $R\left[r^{-1}\right]$-module and the $R\left[r^{-1}\right]$-module $F\left[r^{-1}\right]$ is flat by assumption, so $\operatorname{Tor}_{1}^{R}\left(N\left[r^{-1}\right], F\right)=0$ by Lemma 4.1(b). Furthermore, $\operatorname{ker}\left(l_{N}\right)$ and $\operatorname{coker}\left(l_{N}\right)$ are $r$-torsion $R$-modules.

For any fixed $i \geq 1$ and an $r$-torsion $R$-module $M$, proving that $\operatorname{Tor}_{i}^{R}(M, F)=0$ reduces to showing that $\operatorname{Tor}_{i}^{R}\left(D_{n}, F\right)=0$ for some $R / r R$-modules $D_{n}, n \geq 0$ (by Lemma 4.3). Finally, in view of the assumptions of Lemma 1.10, for any $R / r R$-module $D$ and $i=1$ or 2 one has $\operatorname{Tor}_{i}^{R}(D, F)=0$ by Lemma 4.2(b).

Along the way, we have essentially proved the following result.

Proposition 4.4 Let $R$ be a commutative ring and $r \in R$ be an element. Then all $R$-modules are simply left obtainable from $R / r R$-modules and $R\left[r^{-1}\right]$-modules.

Proof All $r$-torsion $R$-modules are simply left obtainable from $R / r R$-modules by Lemma 4.3. Furthermore, according to the exact sequences (1-2) from the above proof of Lemma 1.10, every $R$-module $N$ is an extension of a torsion $R$-module $\operatorname{ker}\left(l_{N}\right)$ and an $R$-module $\operatorname{im}\left(l_{N}\right)$ obtainable as the kernel of a surjective morphism from an $R\left[r^{-1}\right]$-module $N\left[r^{-1}\right]$ onto an $r$-torsion $R$-module $\operatorname{coker}\left(l_{N}\right)$.

The proof of Toy Main Lemma 1.8 is based on a dual version of Lemma 4.3, which we will now formulate and prove.

We recall (cf. Sects. 0.5 and 1.7) that an $R$-module $C$ is said to be an $r$-contramodule if $\operatorname{Hom}_{R}\left(R\left[r^{-1}\right], C\right)=0=\operatorname{Ext}_{R}^{1}\left(R\left[r^{-1}\right], C\right)$. The full subcategory of $r$-contramodule $R$-modules $R$ - $\bmod _{r \text {-ctra }}$ is closed under the kernels, cokernels, extensions, and infinite products in $R-\bmod$ [9, Proposition 1.1], [24, Theorem 1.2(a)]. The embedding functor $R-\bmod _{r \text {-ctra }} \longrightarrow R$-mod has a left adjoint functor $\Delta_{r}: R-\bmod \longrightarrow R-\bmod _{r \text {-ctra }}$, which can be constructed as follows.

For any complex of $R$-modules $L^{\bullet}$ and an $R$-module $C$, we will use the simplified notation $\operatorname{Ext}_{R}^{i}\left(L^{\bullet}, C\right)$ for the $R$-modules $\operatorname{Hom}_{\mathrm{D}(R \text {-mod })}\left(L^{\bullet}, C[i]\right), i \in \mathbb{Z}$ of morphisms in the derived category $\mathrm{D}(R$-mod $)$. Denote by $K^{\bullet}=K^{\bullet}(R ; r)$ the two-term complex of $R$-modules $R \stackrel{l_{R}}{\longrightarrow}$ $R\left[r^{-1}\right]$, where the term $R$ is placed in the cohomological degree -1 and the term $R\left[r^{-1}\right]$ is placed in the cohomological degree 0 .

According to [24, Theorem 6.4 and Remark 6.5], we have a natural isomorphism $\Delta_{r}(C)=$ $\operatorname{Ext}_{R}^{1}\left(K^{\bullet}, C\right)$ for any $R$-module $C$.

Lemma 4.5 Any $r$-contramodule $R$-module is simply right obtainable from $R / r R$-modules (viewed as $R$-modules via the restriction of scalars). 
Proof This result goes back to [24, proof of Theorem 9.5], where a similar assertion was essentially proved for a finitely generated ideal (rather than just a single element) in a commutative ring.

As one has $\Delta_{r}(C)=C$ for any $r$-contramodule $R$-module $C$, it suffices to show that the $R$-module $\Delta_{r}(A)$ is simply right obtainable from $R / r R$-modules for any $R$-module $A$. For any $R$-module $A$, consider two projective systems of $R$-modules (indexed by the positive integers):

$$
A / r A \longleftarrow A / r^{2} A \longleftarrow A / r^{3} A \longleftarrow-\cdots,
$$

where the maps $A / r^{n+1} A \longrightarrow A / r^{n} A$ are the natural surjections, and

$$
r A \longleftarrow r^{2} A \longleftarrow-r^{3} A \longleftarrow \cdots,
$$

where the maps ${ }_{r^{n+1}} A \longrightarrow r^{n} A$ are provided by the operator of multiplication with $r$.

Sublemma 4.6 Let $R$ be a commutative ring and $r \in R$ be an element. Then for any $R$-module $A$ there is a natural short exact sequence of $R$-modules

$$
0 \longrightarrow \lim _{n \geq 1}^{1} r^{n} A \longrightarrow \Delta_{r}(A) \longrightarrow \lim _{n \geq 1} A / r^{n} A \longrightarrow 0 .
$$

Proof This is [24, Lemma 6.7]. Essentially, the reason is that the complex $K^{\bullet}$ is the inductive limit of the complexes $R \stackrel{r^{n}}{\longrightarrow} R$ over $n \geq 1$, so the complex $\mathbb{R} \operatorname{Hom}_{R}\left(K^{\bullet}, A\right)$ is the homotopy projective limit of the complexes $A \stackrel{r^{n}}{\longrightarrow} A$.

We denote the $r$-adic completion $\lim _{n \geq 1} A / r^{n} A$ of an $R$-module $A$ by $\Lambda_{r}(A)$. Sublemma 4.6 describes the kernel of the natural surjective morphism $\Delta_{r}(A) \longrightarrow \Lambda_{r}(A)$.

Sublemma 4.7 Let $R$ be a commutative ring and $r \in R$ be an element. Let $D_{1} \longleftarrow D_{2} \longleftarrow$ $D_{3} \longleftarrow \cdots$ be a projective system of $R$-modules such that $D_{n}$ is an $R / r^{n} R$-module for every $n \geq 1$. Then the $R$-modules (a) $\lim _{n} D_{n}$ and (b) $\lim _{n}^{1} D_{n}$ are simply right obtainable from $R / r R$-modules.

Proof First of all we notice that, for any $n \geq 2$, any $R / r^{n} R$-module is simply right obtainable (as a finitely iterated extension) from $R / r R$-modules.

Part (a): denote by $D_{n}^{\prime} \subset D_{n}$ the image of the projection map $\lim _{m} D_{m} \longrightarrow D_{n}$. Then we have $\lim _{n} D_{n}=\lim _{n} D_{n}^{\prime}$, and the maps $D_{n+1}^{\prime} \longrightarrow D_{n}^{\prime}$ are surjective. Hence, the $R$-module $\lim _{n} \overleftarrow{D}_{n}$ is a infinitely iterated extension of the $R$-modules $D_{1}^{\prime}$ and $\operatorname{ker}\left(D_{n+1}^{\prime} \rightarrow D_{n}^{\prime}\right), n \geq 1$. The former is, obviously, an $R / r R$-module, and the latter are $\left(R / r^{n+1} R\right)$-modules.

Part (b): by the definition of $\lim _{n \geq 1}^{1} D_{n}$, we have an exact sequence

$$
0 \longrightarrow \lim _{n \geq 1} D_{n} \longrightarrow \prod_{n \geq 1} D_{n} \longrightarrow \prod_{n \geq 1} D_{n} \longrightarrow \lim _{n \geq 1}^{1} D_{n} \longrightarrow 0 .
$$

Hence, the $R$-module $\lim _{n}^{1} D_{n}$ is obtainable from the $R$-modules $\lim _{n} D_{n}$ and $\prod_{n} D_{n}$ by two passages to the cokernel of an injective morphism.

The assertion of Lemma 4.5 follows from Sublemmas 4.6 and 4.7(a-b).

We recall (cf. Sect. 0.5 ) that an $R$-module $C$ is said to be $r$-contraadjusted if $\operatorname{Ext}_{R}^{1}\left(R\left[r^{-1}\right], C\right)=0$. The following result is a dual version of Proposition 4.4.

Toy Main Proposition 4.8 Let $R$ be a commutative ring and $r \in R$ be an element. Then an $R$ module $C$ is $r$-contraadjusted if and only if it is simply right obtainable from $R / r R$-modules and $R\left[r^{-1}\right]$-modules. 
Proof "If": denote by $\mathrm{E} \subset R$-mod the class of all $R / r R$-modules and $R\left[r^{-1}\right]$-modules (viewed as $R$-modules via the restriction of scalars). All the $R$-modules from $\mathrm{E}$ are $r$ contraadjusted, so one has $R\left[r^{-1}\right] \in \mathrm{F}={ }^{\perp} \geq 1 \mathrm{E}$. By Lemma 3.4, it follows that all the $R$-modules simply right obtainable from $\mathrm{E}$ belong to $\mathrm{F}^{\perp_{\geq 1}} \subset\left\{R\left[r^{-1}\right]\right\}^{\perp_{\geq 1}}$, i.e., they are $r$-contraadjusted.

"Only if": there is a distinguished triangle

$$
R \longrightarrow R\left[r^{-1}\right] \longrightarrow K^{\bullet} \longrightarrow R[1]
$$

in the derived category $\mathrm{D}(R-\mathrm{mod})$. Applying the functor $\operatorname{Hom}_{\mathrm{D}(R-\bmod )}(-, C[*])$, we obtain a 5-term exact sequence

$$
\begin{aligned}
0 \longrightarrow \operatorname{Hom}_{R}\left(R\left[r^{-1}\right] / R, C\right) \longrightarrow \operatorname{Hom}_{R}\left(R\left[r^{-1}\right], C\right) \longrightarrow C \\
\longrightarrow \Delta_{r}(C) \longrightarrow \operatorname{Ext}_{R}^{1}\left(R\left[r^{-1}\right], C\right) \longrightarrow 0,
\end{aligned}
$$

where $R\left[r^{-1}\right] / R$ is a shorthand notation for the cokernel of the map $l_{R}: R \longrightarrow R\left[r^{-1}\right]$, the derived category Hom module $\operatorname{Ext}_{R}^{0}\left(K^{\bullet}, C\right)=\operatorname{Hom}_{\mathrm{D}(R-\bmod )}\left(K^{\bullet}, C\right)$ is isomorphic to $\operatorname{Hom}_{R}\left(R\left[r^{-1}\right] / R, C\right)$, and $\operatorname{Ext}_{R}^{1}\left(K^{\bullet}, C\right)=\Delta_{r}(C)$.

When the $R$-module $C$ is $r$-contraadjusted, the rightmost term vanishes, so we are reduced to a 4-term exact sequence

$$
0 \longrightarrow \operatorname{Hom}_{R}\left(R\left[r^{-1}\right] / R, C\right) \longrightarrow \operatorname{Hom}_{R}\left(R\left[r^{-1}\right], C\right) \longrightarrow C \longrightarrow \Delta_{r}(C) \longrightarrow 0 \text {. }
$$

Now $\operatorname{Hom}_{R}\left(R\left[r^{-1}\right], C\right)$ is an $R\left[r^{-1}\right]$-module, $\Delta_{r}(C)$ is an $r$-contramodule $R$-module (computed in Sublemma 4.6), and $\operatorname{Hom}_{R}\left(R\left[r^{-1}\right] / R, C\right)$ is also an $r$-contramodule (by [24, Lemma 6.1(a)]). In fact, $M=R\left[r^{-1}\right] / R$ is an $r$-torsion $R$-module, so $\operatorname{Hom}_{R}(M, C)$ is the projective limit of the sequence of $R / r^{n} R$-modules $\operatorname{Hom}_{R}\left(r^{n} M, C\right)$ (cf. Sublemma 4.7(a)).

To sum up, the $R$-module $C$ is obtainable from two $r$-contramodule $R$-modules and one $R\left[r^{-1}\right]$-module using one passage to the cokernel of an injective morphism and one passage to an extension of two modules. In view of Lemma 4.5, the assertion of the toy main proposition follows.

Proof of Toy Main Lemma 1.8 The "only if" assertion holds, because the functors $F \longmapsto$ $F\left[r^{-1}\right]$ and $F \longmapsto F / r F$ preserve transfinitely iterated extensions, in the sense of the inductive limit, of flat $R$-modules, and both of them take the $R$-module $R\left[r^{-1}\right]$ to a free module (with 1 or 0 generators) over the respective ring. The "if" assertion is the nontrivial part.

To show that the $R$-module $F$ is $r$-very flat, we need to check that $\operatorname{Ext}_{R}^{1}(F, C)=0$ for all $r$-contraadjusted $R$-modules $C$. As in the proof of Toy Main Proposition 4.8, we denote by $\mathrm{E} \subset R$-mod the class of all $R / r R$-modules and $R\left[r^{-1}\right]$-modules.

By Lemma 4.1(a), it follows from the assumptions of flatness of the $R$-module $F$ and projectivity of the $R / r R$-module $F / r F$ and the $R\left[r^{-1}\right]$-module $F\left[r^{-1}\right]$ that $\operatorname{Ext}_{R}^{i}(F, E)=0$ for all $E \in \mathrm{E}$ and $i \geq 1$. So $F \in{ }^{\perp_{\geq 1}} \mathrm{E}$. By Toy Main Proposition 4.8, the $R$-module $C$ is simply right obtainable from $\mathrm{E}$. By Lemma 3.4 , it follows that $C \in\left({ }^{\perp_{\geq 1}} \mathrm{E}\right)^{\perp_{\geq 1}} \subset\{F\}^{\perp_{\geq 1}}$, and hence, $\operatorname{Ext}_{R}^{1}(F, C)=0$. 


\section{Contramodule approximation sequences}

The proofs of Main Lemmas 1.4 and 1.7 are based on the constructions of certain short exact sequences of $r$-contramodule $R$-modules, which are called the approximation sequences. In Sect. 5.1, we present the related general background material about cotorsion theories and approximation sequences in abelian categories. Then we proceed to construct the needed short exact sequences in Sects. 5.2, 5.3, and 5.6. The argument deducing the main lemmas from the existence of approximation sequences is based on the discussion of separated $r$-contramodule $R$-modules in Sect. 5.4; the important category of quotseparated $r$-contramodule $R$-modules is introduced in Sect. 5.5.

\subsection{Cotorsion theories in abelian categories}

Let $A$ be an abelian category and $F, C \subset A$ be two classes of objects in $A$. We will denote by $\mathrm{F}^{\perp_{1}} \subset \mathrm{A}$ the class of all objects $C \in \mathrm{A}$ such that $\operatorname{Ext}_{\mathrm{A}}^{1}(F, C)=0$ for all $F \in \mathrm{F}$, and by ${ }^{{ }_{1}} \mathrm{C} \subset \mathrm{A}$ the class of all objects $F \in \mathrm{A}$ such that $\operatorname{Ext}_{\mathrm{A}}^{1}(F, C)=0$ for all $C \in \mathrm{C}$.

A pair of classes of objects $(F, C)$ in an abelian category $A$ is called a cotorsion theory (or a cotorsion pair) if $C=F^{\perp_{1}}$ and $F={ }^{\perp_{1}} C$ (see, e.g., [30, Section 5] or [24, Section 8]). A cotorsion theory $(\mathrm{F}, \mathrm{C})$ is called complete if for every object $X \in \mathrm{A}$ there exist short exact sequences in the category $A$

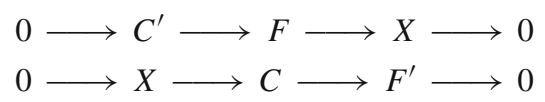

with some objects $F, F^{\prime} \in \mathrm{F}$ and $C, C^{\prime} \in \mathrm{C}$. The short exact sequences (5-6) are called approximation sequences for an object $X \in A$ with respect to the cotorsion theory $(\mathrm{F}, \mathrm{C})$. The morphism $F \longrightarrow X$ in a short exact sequence (5) is called a special $\mathrm{F}$-precover of an object $X$, while the morphism $X \longrightarrow C$ in a short exact sequence (6) is called a special C-preenvelope of $X$.

Conversely, let $\mathrm{F}$ and $\mathrm{C} \subset \mathrm{A}$ be two classes of objects such that $\operatorname{Ext}_{\mathrm{A}}^{1}(F, C)=0$ for all $F \in \mathrm{F}$ and $C \in \mathrm{C}$, and short exact sequences (5-6) exist for all objects $X \in \mathrm{A}$. Assume that the classes of objects $F$ and $C$ are closed with respect to the passages to direct summands in $A$. Then the pair of classes of objects $(F, C)$ is a (complete) cotorsion theory in A.

A complete cotorsion theory $(F, C)$ in $A$ is said to be hereditary if one of the following equivalent conditions holds [30, Lemma 6.17]:

(i) $\operatorname{Ext}_{\mathrm{A}}^{i}(F, C)=0$ for all $F \in \mathrm{F}, C \in \mathrm{C}$, and $i \geq 1$;

(ii) $\operatorname{Ext}_{\mathrm{A}}^{2}(F, C)=0$ for all $F \in \mathrm{F}$ and $C \in \mathrm{C}$;

(iii) the full subcategory $F \subset A$ is closed under the passages to the kernels of epimorphisms;

(iv) the full subcategory $C \subset A$ is closed under the passages to the cokernels of monomorphisms.

So, in a hereditary complete cotorsion theory, one has $C=F^{\perp_{\geq 1}}$ and $F={ }^{\perp_{\geq 1}} \mathrm{C}$ (in the notation of Sect. 3).

Let $S \subset A$ be a class of objects. Clearly, the pair of classes $F=S^{\perp_{1}}$ and $C={ }^{\perp_{1}} \mathrm{~F}$ is a cotorsion theory in A. One says that the cotorsion theory $(F, C)$ is generated by the class of objects $S \subset A$.

Any complete cotorsion theory generated by a class of objects of projective dimension not exceeding 1 in an abelian category A is hereditary (as the above condition (iv) is clearly satisfied). Moreover, in such a cotorsion theory the class $C$ is closed under the passages to 
arbitrary quotient objects, while the class $F$ consists of objects of projective dimension not exceeding 1.

A classical result of the paper [6, Theorem 10] claims that, in the abelian category $A=$ $R$-mod of modules over an associative ring $R$, any cotorsion theory generated by a set of objects is complete. Among many applications of this result, it allows to prove completeness of the following cotorsion theories in $R$-mod, which are of relevance to us.

A left module $C$ over an associative ring $R$ is said to be cotorsion [7] (cf. Sect. 0.10) if $\operatorname{Ext}_{R}^{1}(F, C)=0$ for all flat left $R$-modules $C$. The pair of full subcategories (flat left $R$-modules, cotorsion left $R$-modules) forms a complete cotorsion theory in $R$-mod [4, Proposition 2], which is called the flat cotorsion theory. The flat cotorsion theory in $R$-mod is also hereditary (as the condition (iii) is satisfied).

The definitions of very flat and contraadjusted modules over a commutative ring $R$ are given in Sect. 0.5. The pair of full subcategories (very flat $R$-modules, contraadjusted $R$ modules) forms a complete cotorsion theory in $R-\bmod [22$, Section 1.1], which is called the very flat cotorsion theory. The very flat cotorsion theory is hereditary, because it is generated by a set of modules of projective dimension not exceeding 1 (namely, the $R$-modules $R\left[r^{-1}\right.$, $r \in R)$.

In this section, we are interested in certain (hereditary complete) cotorsion theories in the abelian category $\mathrm{A}=R$ - $\bmod _{r \text {-ctra }}$ of $r$-contramodule $R$-modules, where $R$ is a commutative ring and $r \in R$ is an element. One thing one can say about the abelian category $R$ - $\bmod _{r \text {-ctra }}$ is that it is locally presentable, or more precisely, locally $\aleph_{1}$-presentable [26, Examples 4.1]. The following result from the paper [26] provides a generalization of [6, Theorem 10] to locally presentable abelian categories.

Theorem 5.1 Let A be a locally presentable abelian category, $\mathrm{S} \subset \mathrm{A}$ be a set of objects, and $(\mathrm{F}, \mathrm{C})$ be the cotorsion theory generated by $\mathrm{S}$ in A. Assume that every object of $\mathrm{A}$ is a quotient object of an object from $\mathrm{F}$ and a subobject of an object from $\mathrm{C}$. Then the cotorsion theory $(\mathrm{F}, \mathrm{C})$ in the category $\mathrm{A}$ is complete.

Proof This is [26, Corollary 3.6] (see [26, Theorem 4.8] for further details).

\subsection{Flat cotorsion theory in $\boldsymbol{r}$-contramodule $\boldsymbol{R}$-modules for a Noetherian commutative ring $R$}

In this section, we will consider $r$-contramodule $R$-modules that are flat, cotorsion, or contraadjusted as $R$-modules. To emphasize this aspect, we will call such $r$-contramodule $R$-modules $R$-flat, $R$-cotorsion, or $R$-contraadjusted.

The aim of this section is to present a sketch of proof of the following theorem (which is a particular case of the results of [22, Section C.2]).

Theorem 5.2 Let $R$ be a Noetherian ring and $r \in R$ be an element. Then the pair of full subcategories ( $R$-flat $r$-contramodule $R$-modules, $R$-cotorsion $r$-contramodule $R$-modules) is a hereditary complete cotorsion theory in the abelian category $R-\bmod _{r \text {-ctra }}$.

The idea of the proof is to produce approximation sequences for an $r$-contramodule $R$ module from its approximation sequences in the flat cotorsion theory in the category of arbitrary $R$-modules.

Let $R$ be a commutative ring and $r \in R$ be an element. For any $R$-module $M$, we denote by $b_{M}$ the $R$-module morphism $\operatorname{Hom}_{R}\left(R\left[r^{-1}\right], M\right) \longrightarrow M$ obtained by applying the functor $\operatorname{Hom}_{R}(-, M)$ to the $R$-module morphism $R \longrightarrow R\left[r^{-1}\right]$. According to 
the exact sequence (3) from the proof of Toy Main Proposition 4.8, one has $\operatorname{ker}\left(b_{M}\right)=$ $\operatorname{Hom}_{R}\left(R\left[r^{-1}\right] / R, M\right)$.

Let $h_{r}(M) \subset M$ denote the image of the morphism $b_{M}$. Equivalently, $h_{r}(M)$ is the maximal $r$-divisible $R$-submodule in $M$. According to the exact sequence (4), for any $r$ contraadjusted $R$-module $K$ the adjunction morphism $K \longrightarrow \Delta_{r}(K)$ is surjective with the kernel $h_{r}(K)$, so we have $\Delta_{r}(K)=K / h_{r}(K)$.

We recall that the class of $r$-contraadjusted $R$-modules is closed under the passages to arbitrary quotient objects, while the class of cotorsion $R$-modules is closed under the cokernels of injective morphisms.

Lemma 5.3 Let $R$ be a commutative ring and $r, s \in R$ be two elements. Then

(a) for any $r$-contraadjusted $R$-module $K$, the $R$-module $h_{r}(K)$ is $r$-contraadjusted;

(b) for any ( $r s)$-contraadjusted $R$-module $K$, the $R$-module $h_{r}(K)$ is s-contraadjusted;

(c) for any cotorsion $R$-module $K$, the $R$-module $\operatorname{Hom}_{R}\left(R\left[r^{-1}\right], K\right)$ is cotorsion.

Proof Part (b): the $R$-module $\operatorname{Hom}_{R}\left(R\left[r^{-1}\right], K\right)$ is $s$-contraadjusted for any $(r s)$ contraadjusted $R$-module $K$ by Lemma 8.6 below (cf. [22, proof of Lemma 1.2.1]); hence, its quotient $R$-module $h_{r}(C)$ is also $s$-contraadjusted. Part (a) is part (b) for $r=s$. Part (c): the $R$-module $\operatorname{Hom}_{R}(F, K)$ is cotorsion for any flat $R$-module $F$ and cotorsion $R$-module $K$ [22, Lemma 1.3.2(a)].

We refer to Sect. 1.4 for the definition of what it means that an $R$-module has bounded $r$-torsion.

Lemma 5.4 Let $R$ be a Noetherian commutative ring and $r \in R$ be an element. Then any flat $R$-module has bounded $r$-torsion.

Proof This is a particular case of [22, Lemma C.2.1], which can be also obtained as a particular case of Lemma 5.8 .

The following lemma plays a key role.

Lemma 5.5 Let $R$ be a Noetherian commutative ring and $r \in R$ be an element. Let $F$ be a flat $r$-contraadjusted $R$-module $F$. Then the $R$-module $\Delta_{r}(F)=F / h_{r}(F)$ is also flat.

Proof There are many ways to prove this basic result. In particular, one can deduce it from [22, Lemmas 1.6.2 and C.2.1], as explained in [22, proof of Corollary C.2.3(a)]. Alternatively, one can observe that the $R$-module $\Delta_{r}(F)$ is flat for any flat $R$-module $F$, which is a particular case of [24, Corollary 10.4(b)].

Now we can produce the promised approximation sequences in $R-\bmod _{r \text {-ctra }}$.

Lemma 5.6 Let $R$ be a Noetherian commutative ring and $r \in R$ be an element. Let $C$ be an $r$-contramodule $R$-module, and let $0 \longrightarrow K \longrightarrow F \longrightarrow C \longrightarrow 0$ be a short exact sequence of $R$-modules with a flat $R$-module $F$ and an $r$-contraadjusted $R$-module $K$. Then $0 \longrightarrow K / h_{r}(K) \longrightarrow F / h_{r}(F) \longrightarrow C \longrightarrow 0$ is a short exact sequence of $r$-contramodule $R$-modules with a flat $R$-module $F / h_{r}(F)$. If $K$ is a cotorsion $R$-module, then so are the $R$-modules $h_{r}(K)$ and $K / h_{r}(K)$.

Proof This is [22, Lemma C.2.6]. By Lemma 5.4, we have $\operatorname{Hom}_{R}\left(R\left[r^{-1}\right] / R, F\right)=0$, and consequently $\operatorname{Hom}_{R}\left(R\left[r^{-1}\right] / R, K\right)=0$. Therefore, $h_{r}(K)=\operatorname{Hom}_{R}\left(R\left[r^{-1}\right], K\right)$ and 
$h_{r}(F)=\operatorname{Hom}_{R}\left(R\left[r^{-1}\right], F\right)$. Since $C$ is an $r$-contramodule $R$-module, it follows that the morphism $K \longrightarrow F$ induces an isomorphism $h_{r}(K) \simeq h_{r}(F)$. Thus, the short sequence $0 \longrightarrow K / h_{r}(K) \longrightarrow F / h_{r}(F) \longrightarrow C \longrightarrow 0$ is exact.

The $R$-module $F$ is $r$-contraadjusted as an extension of two $r$-contraadjusted $R$-modules $C$ and $K$. The $R$-modules $K$ and $F$ being $r$-contraadjusted, it follows that the $R$-modules $K / h_{r}(K)$ and $F / h_{r}(F)$ are $r$-contramodules. The $R$-module $F / h_{r}(F)$ is flat by Lemma 5.5. If the $R$-module $K$ is cotorsion, then the $R$-module $h_{r}(K)$ is cotorsion by Lemma 5.3(c); hence, the $R$-module $K / h_{r}(K)$ is cotorsion as the cokernel of an injective morphism of cotorsion $R$-modules.

Lemma 5.7 Let $R$ be a Noetherian commutative ring and $r \in R$ be an element. Let $C$ be an $r$-contramodule $R$-module, and let $0 \longrightarrow C \longrightarrow K \longrightarrow F \longrightarrow 0$ be a short exact sequence of $R$-modules with a flat $R$-module $F$ and an $r$-contraadjusted $R$-module $K$. Then $0 \longrightarrow C \longrightarrow K / h_{r}(K) \longrightarrow F / h_{r}(F) \longrightarrow 0$ is a short exact sequence of $r$-contramodule $R$-modules with a flat $R$-module $F / h_{r}(F)$. If $K$ is a cotorsion $R$-module, then so are the $R$-modules $h_{r}(K)$ and $K / h_{r}(K)$.

Proof This is [22, Lemma C.2.4]. By Lemma 5.4, we have $\operatorname{Hom}_{R}\left(R\left[r^{-1}\right] / R, F\right)=0$. Since $\operatorname{Hom}_{R}\left(R\left[r^{-1}\right] / R, C\right) \subset \operatorname{Hom}_{R}\left(R\left[r^{-1}\right], C\right)=0$, it follows that $\operatorname{Hom}_{R}\left(R\left[r^{-1}\right] / R, K\right)=0$. The proof of exactness of the short sequence $0 \longrightarrow C \longrightarrow K / h_{r}(K) \longrightarrow F / h_{r}(F) \longrightarrow 0$ finishes as in the proof of Lemma 5.6.

The $R$-module $F$ is $r$-contraadjusted as a quotient module of an $r$-contraadjusted $R$ module $K$. The rest of the argument is the same as in Lemma 5.6.

Proof of Theorem 5.2 One has $\operatorname{Ext}_{R \text { - } \bmod _{r \text {-ctra }}}^{1}(F, C)=0$ for any $R$-flat $r$-contramodule $R$ module $F$ and any $R$-cotorsion $r$-contramodule $R$-module $C$, because $\operatorname{Ext}_{R}^{1}(F, C)=0$ and $R$ - od $_{r \text {-ctra }} \subset R$-mod is a full subcategory closed under kernels, cokernels, and extensions. The approximation sequences (5-6) in $R-\bmod _{r \text {-ctra }}$ can be produced from the similar sequences in $R$-mod using Lemmas 5.6-5.7. It remains to observe that the classes of $R$-flat $r$-contramodule $R$-modules and $R$-cotorsion $r$-contramodule $R$-modules are closed under direct summands. The flat cotorsion theory in $R-\bmod _{r \text {-ctra }}$ is hereditary, because the conditions (iii) and/or (iv) from Sect. 5.1 are satisfied (as the flat cotorsion theory in $R$-mod is hereditary).

\subsection{Very flat cotorsion theory in the bounded torsion case}

The notion of a left contramodule over a complete, separated topological associative ring $\mathfrak{R}$ with a base of neighborhoods of zero formed by open right ideals was introduced in [19, Remark A.3], [21, Section 1.2], and studied in [22, Appendix D], [26, Sections 5-7]. In the context of this paper, we set $\Re=\lim _{n \geq 1} R / r^{n} R$ to be the $r$-adic completion of a commutative ring $R$, endowed with the projective limit (=r-adic) topology. We denote the abelian category of $\mathfrak{R}$-contramodules by $\mathfrak{R}$-contra.

In this section, we are interested in commutative rings $R$ with an element $r \in R$ such that the $r$-torsion in $R$ is bounded (cf. Sect. 1.4).

Lemma 5.8 Let $R$ be a commutative ring and $r \in R$ be an element such that the $r$-torsion in $R$ is bounded. Then the $R$-torsion in any flat $R$-module is bounded, too.

Proof Let $m \geq 1$ be an integer such that one has $r^{m} x=0$ for any $r$-torsion element $x \in R$. Using the Govorov-Lazard description of flat $R$-modules as filtered inductive limits 
of projective ones, one easily shows that for any flat $R$-module $F$ and an $r$-torsion element $y \in F$ one also has $r^{m} y=0$.

The Noetherian particular case of the following result goes back to [21, Theorem B.1.1]. For a generalization to arbitrary commutative rings $R$ with an element $r$, see Theorem 5.20.

Theorem 5.9 Let $R$ be a commutative ring and $r \in R$ be an element such that the $r$-torsion in $R$ is bounded. Then the forgetful functor $\mathfrak{R}$-contra $\longrightarrow R$-mod induces an equivalence of abelian categories $\mathfrak{R}$-contra $\simeq R$ - $\bmod _{r \text {-ctra }}$.

Proof This is [25, Example 2.2(5)]. Essentially, both $\mathfrak{R}$-contra and $R$ - $\bmod _{r \text {-ctra }}$ are isomorphic to the category of algebras/modules over the additive monad $X \longmapsto \Delta_{r}(R[X])=$ $\Lambda_{r}(R[X])$ on the category of sets, where $R[X]$ denotes the free $R$-module generated by a set $X$ (cf. Sublemma 4.6).

So, in the bounded torsion case, "contramodules over the topological ring $\mathfrak{R}$ " is just another name for $r$-contramodule $R$-modules. The point is that this interpretation of $r$-contramodule $R$-modules allows to apply the results of [26, Section 7] about cotorsion theories in $\Re$-contra.

An $\Re$-contramodule $F$ is called flat if the $R / r^{n} R$-module $F / r^{n} F$ is flat for every $n \geq 1$ [22, Section D.1], [26, Sections 5-6]. Unlike in the Noetherian case of Sect. 5.2, there is no claim that flat $\mathfrak{R}$-contramodules are flat $R$-modules here.

In fact, when the $r$-torsion in $R$ is bounded, an $\mathfrak{R}$-contramodule or an $r$-contramodule $R$-module is the same thing as a cohomologically $I$-adically complete $R$-module in the sense of [18,32], where $I=(r) \subset R$ is the principal ideal generated by the element $r \in R$ [23, Section 0.9]. Furthermore, in the same assumption, an $\mathfrak{R}$-contramodule is flat if and only if it is an $I$-adically flat $R$-module in the sense of the paper [33] (see [33, Theorems 4.3 and 6.9] and [24, Lemma 10.1]). The counterexample in [33, Theorem 7.2] shows that a flat $\Re$-contramodule does not need to be a flat $R$-module.

Theorem 5.10 The cotorsion theory generated by any set of flat $\mathfrak{R}$-contramodules is complete in $\Re$-contra.

Proof This is a particular case of [26, Corollary 7.11].

Lemma 5.11 For any $R$-module $A$ and any integer $n \geq 1$, the adjunction morphism $A \longrightarrow$ $\Delta_{r}(A)$ induces an isomorphism $A / r^{n} A \simeq \Delta_{r}(A) / r^{n} \Delta_{r}(A)$.

Proof All $R / r^{n} R$-modules are $r$-contramodule $R$-modules. Since the functor $\Delta_{r}$ is left adjoint to the embedding $R-\bmod _{r \text {-ctra }} \longrightarrow R$-mod, for any $R / r^{n} R$-module $D$ we have

$$
\begin{aligned}
\operatorname{Hom}_{R / r^{n}}\left(A / r^{n} A, D\right) & =\operatorname{Hom}_{R}(A, D) \\
=\operatorname{Hom}_{R}\left(\Delta_{r}(A), D\right) & =\operatorname{Hom}_{R / r^{n} R}\left(\Delta_{r}(A) / r^{n} \Delta_{r}(A), D\right),
\end{aligned}
$$

implying the desired isomorphism.

Consider the set of all the $r$-contramodule $R$-modules $\Delta_{r}\left(R\left[s^{-1}\right]\right)$, where $s \in R$. One has $\Delta_{r}\left(R\left[s^{-1}\right]\right) / r^{n} \Delta_{r}\left(R\left[s^{-1}\right]\right)=R\left[s^{-1}\right] / r^{n} R\left[s^{-1}\right]=\left(R / r^{n} R\right)\left[s^{-1}\right]$ by Lemma 5.11, so $\Delta_{r}\left(R\left[s^{-1}\right]\right)$ is a flat $\mathfrak{R}$-contramodule.

The cotorsion theory $(\mathrm{F}, \mathrm{C})$ generated by the set of all $r$-contramodule $R$-modules $\Delta_{r}\left(R\left[s^{-1}\right]\right)$ is called the very flat cotorsion theory in $R$ - $\bmod _{r \text {-ctra }}$. The objects from the class $\mathrm{F}$ are called the very flat $r$-contramodule $R$-modules, and the objects of the class $C$ are called the contraadjusted $r$-contramodule $R$-modules. 
Once again, there is no claim that very flat $r$-contramodule $R$-modules are very flat $R$ modules (even for a Noetherian ring $R$; $\mathrm{cf}$. [22, Section C.3]). On the other hand, the following results hold.

Proposition 5.12 Let $R$ be a commutative ring and $r \in R$ be an element such that the $r$-torsion in $R$ is bounded. Then all very flat $r$-contramodule $R$-modules are flat $\mathfrak{R}$-contramodules.

Proof This follows from the existence of the flat cotorsion theory in $\Re$-contra; see [26, Corollary 7.8]. Alternatively, one can apply [26, Theorem 4.8(a,d)] to show that an $r$-contramodule $R$-module is very flat if and only if it is a direct summand of a transfinitely iterated extension of the objects $\Delta_{r}\left(R\left[s^{-1}\right]\right)$ in the category $R-\bmod _{r \text {-ctra }}$ (in the sense of [26, Definition 4.3]). All such transfinitely iterated extensions are flat $\Re$-contramodules by [26, Lemma 5.6 and Corollary 7.1(b)].

Theorem 5.13 Let $R$ be a commutative ring and $r \in R$ be an element such that the $r$-torsion in $R$ is bounded. Then an $r$-contramodule $R$-module is contraadjusted if and only if it is contraadjusted as an R-module.

Proof Recall that the functor $\Delta_{r}$ can be computed as $\Delta_{r}(A)=\operatorname{Ext}_{R}^{1}\left(K^{\bullet}, A\right)$, where $K^{\bullet}=$ $K^{\bullet}(R ; r)$ denotes the two-term complex $R \longrightarrow R\left[r^{-1}\right]$ (see Sect. 4). Now let $0 \longrightarrow C \longrightarrow$ $B \longrightarrow A \longrightarrow 0$ be a short exact sequence of $R$-modules. Applying the functor $\operatorname{Ext}_{R}^{*}\left(K^{\bullet},-\right)$, we obtain a long exact sequence

$\cdots \longrightarrow \operatorname{Hom}_{R}\left(R\left[r^{-1}\right] / R, A\right) \longrightarrow \Delta_{r}(C) \longrightarrow \Delta_{r}(B) \longrightarrow \Delta_{r}(A) \longrightarrow 0$.

Let $C$ be an $r$-contramodule $R$-module. Suppose that we have a short exact sequence of $R$-modules

$$
0 \longrightarrow C \longrightarrow B \longrightarrow R\left[s^{-1}\right] \longrightarrow 0 .
$$

Applying the functor $\Delta_{r}$, we get a short exact sequence

$$
0 \longrightarrow C \longrightarrow \Delta_{r}(B) \longrightarrow \Delta_{r}\left(R\left[s^{-1}\right]\right) \longrightarrow 0,
$$

because $\Delta_{r}(C)=C$ and $\operatorname{Hom}_{R}\left(R\left[r^{-1}\right] / R, R\left[s^{-1}\right]\right)=0$ by Lemma 5.8. There is a natural (adjunction) morphism from the short exact sequence (8) into the short exact sequence (9); hence, it follows that the sequence (8) is the pullback of the sequence (9) with respect to the morphism $R\left[s^{-1}\right] \longrightarrow \Delta_{r}\left(R\left[s^{-1}\right]\right)$.

Conversely, given a short exact sequence of $r$-contramodule $R$-modules

$$
0 \longrightarrow C \longrightarrow B^{\prime} \longrightarrow \Delta_{r}\left(R\left[s^{-1}\right]\right) \longrightarrow 0,
$$

one can take the pullback with respect to the morphism $R\left[s^{-1}\right] \longrightarrow \Delta_{r}\left(R\left[s^{-1}\right]\right)$ in order to produce a short exact sequence (8). Then the adjunction provides an isomorphism from the exact sequence (9) into the exact sequence (10).

We have constructed an isomorphism of the Ext modules

$$
\operatorname{Ext}_{R}^{1}\left(R\left[s^{-1}\right], C\right) \simeq \operatorname{Ext}_{R-\bmod _{r-\mathrm{ctra}}}^{1}\left(\Delta_{r}\left(R\left[s^{-1}\right]\right), C\right)
$$

for any $r$-contramodule $R$-module $C$, implying the assertion of the theorem.

Using sequence (7), one can also show that the objects $\Delta_{r}\left(R\left[s^{-1}\right]\right)$ have projective dimen-

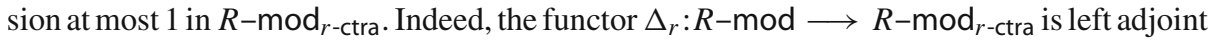
to an exact functor, so it takes projectives to projectives. Applying it to a projective resolution $0 \longrightarrow Q \longrightarrow P \longrightarrow R\left[s^{-1}\right] \longrightarrow 0$ of the $R$-module $R\left[s^{-1}\right]$, one obtains a projective resolution $0 \longrightarrow \Delta_{r}(Q) \longrightarrow \Delta_{r}(P) \longrightarrow \Delta_{r}\left(R\left[s^{-1}\right]\right) \longrightarrow 0$ of the $r$-contramodule $R$-module $\Delta_{r}\left(R\left[s^{-1}\right]\right)$. 
Remark 5.14 Alternatively, one could define a very flat $\mathfrak{R}$-contramodule as an $\mathfrak{R}$ contramodule $F$ such that the $R / r^{n} R$-module $F / r^{n} F$ is very flat for all $n \geq 1$. This also leads to a complete cotorsion theory in $\Re$-contra [26, Example 7.12(3)]. In fact, such a definition of a very flat $r$-contramodule $R$-module for a commutative ring $R$ with bounded $r$-torsion is equivalent to the above, so the two "very flat cotorsion theories in $R-\bmod _{r \text {-ctra" }}$ coincide. This can be shown by comparing Theorem 5.13 with the results of [22, Section D.4], or more specifically [22, Corollary D.4.8] (implying that the classes of contraadjusted objects in the two "very flat cotorsion theories" coincide). We will discuss the theory of [22, Section D.4] below in Sect. 5.6.

\subsection{Separated $r$-contramodule $R$-modules}

Let $R$ be a commutative ring and $r \in R$ be an element. An $R$-module $C$ is said to be $r$-complete, if the natural map

$$
\lambda_{r, C}: C \longrightarrow \Lambda_{r}(C)=\lim _{n \geq 1} C / r^{n} C
$$

is surjective, and $r$-separated, if the map $\lambda_{r, C}$ is injective (cf. Sect. 1.7).

Clearly, an $R$-module $C$ is $r$-separated if and only if the intersection $\bigcap_{n \geq 1} r^{n} C \subset C$ vanishes. It follows that any $R$-submodule of an $r$-separated $R$-module is $r$-separated.

Any $r$-contraadjusted $R$-module is $r$-complete [24, Theorem 2.3(a)]. Any $r$-separated and $r$-complete $R$-module is an $r$-contramodule [24, Theorem 2.4(b)]. However, an $r$-contramodule $R$-module does not need to be $r$-separated [18, Example 4.33], [24, Example 2.7(1)]. The kernel $\bigcap_{n \geq 1} r^{n} C \subset C$ of the $r$-completion morphism $\lambda_{r, C}$ for an $r$-contramodule $R$-module $C$ is computed in Sublemma 4.6.

The following corollaries pick out the aspects of the results of Sects. 5.2-5.3 relevant for the proofs of Main Lemmas 1.4 and 1.7 in Sect. 7. We recall that the terms " $R$-flat" and " $R$ contraadjusted" are being used as shorthands for "flat as an $R$-module" and "contraadjusted as an $R$-module."

Corollary 5.15 Let $R$ be a Noetherian commutative ring, $r \in R$ be an element, and $C$ be an $R$-contraadjusted $r$-contramodule $R$-module. Then the $R$-module $C$ can be presented as the cokernel of an injective morphism of $R$-contraadjusted $r$-separated $r$-complete $R$-modules.

Proof By Theorem 5.2, or more specifically by Lemma 5.6, there exists a short exact sequence of $r$-contramodule $R$-modules $0 \longrightarrow K \longrightarrow F \longrightarrow C \longrightarrow 0$, where the $R$-module $F$ is flat and the $R$-module $K$ is cotorsion. What is important for us is that the $R$-module $K$ is contraadjusted; since the $R$-module $C$ is contraadjusted by assumption, it follows that the $R$-module $F$ is contraadjusted, too.

Furthermore, any $R$-flat $r$-contramodule $R$-module is $r$-separated [24, Corollary 10.3(b)]. The $R$-module $F$ being $r$-separated, it follows that its submodule $K$ is $r$-separated, too. Thus $K \longrightarrow F$ is an injective morphism of $R$-contraadjusted $r$-separated $r$-contramodules with the cokernel $C$.

Corollary 5.16 Let $R$ be a commutative ring and $r \in R$ be an element such that the $r$-torsion in $R$ is bounded. Then any $R$-contraadjusted $r$-contramodule $R$-module can be presented as the cokernel of an injective morphism of $R$-contraadjusted $r$-separated $r$-complete $R$-modules.

Proof By Theorem 5.10, the very flat cotorsion theory in $\mathfrak{R}$-contra (as defined in Sect. 5.3) is complete. By Theorem 5.9, the categories $\mathfrak{R}$-contra and $R$ - $\bmod _{r \text {-ctra }}$ coincide. Hence, 
for any $r$-contramodule $R$-module $C$ there exists a short exact sequence $0 \longrightarrow K \longrightarrow$ $F \longrightarrow C \longrightarrow 0$, where $K$ is a contraadjusted $r$-contramodule $R$-module and $F$ is a very flat $r$-contramodule $R$-module.

By Theorem 5.13, $K$ is a contraadjusted $R$-module. Assuming that $C$ is a contraadjusted $R$ module, we can conclude that the $R$-module $F$ is contraadjusted, too. By Proposition 5.12, $F$ is a flat $\mathfrak{R}$-contramodule. By [22, Corollary D.1.7] or [26, Corollary 6.15], all flat $\mathfrak{R}$ contramodules are $r$-separated. So the $R$-module $F$ is $r$-separated, and it follows that its submodule $K$ is $r$-separated, too.

Thus, $K \longrightarrow F$ is an injective morphism of $R$-contraadjusted $r$-separated $r$-contramodule $R$-modules with the cokernel $C$.

Remark 5.17 Corollary 5.16 is a generalization of Corollary 5.15, and Corollary 5.16 as it is stated can be, in turn, generalized a bit further, though admittedly not much.

As above, let $h_{r}(M) \subset M$ denote the maximal $r$-divisible submodule in an $R$-module $M$. In particular, $h_{r}(R)$ is an ideal in $R$. Let $C$ be an $r$-contramodule $R$-module. For any element $c \in C$, consider the $R$-module morphism $f_{c}: R \longrightarrow C$ taking 1 to $c$. Since there are no $r$-divisible submodules in $C$, we have $f_{c}\left(h_{r}(R)\right)=0$. Hence, the ideal $h_{r}(R) \subset R$ acts by zero in $C$, so $C$ is an $R / h_{r}(R)$-module.

Furthermore, an $R / h_{r}(R)$-module is contraadjusted if and only if it is contraadjusted as an $R$-module [22, Lemma 1.6.6(a)], and the property of an $R$-module to be an $r$-contramodule only depends on its underlying abelian group structure and the action of the operator $r$ [24, Remark 5.5]. So any $R$-contraadjusted $r$-contramodule $R$-module $C$ is at the same time an $R / h_{r}(R)$-contraadjusted $\bar{r}$-contramodule $R / h_{r}(R)$-module, where $\bar{r} \in R / h_{r}(R)$ denotes the image of the element $r \in R$.

Assume that the $r$-torsion ( $=\bar{r}$-torsion) in $R / h_{r}(R)$ is bounded. Applying Corollary 5.16 to the $R / h_{r}(R)$-module $C$, we conclude that it is the cokernel of an injective morphism of $R / h_{r}(R)$-contraadjusted $\bar{r}$-separated $\bar{r}$-contramodule $R / h_{r}(R)$-modules, or, which is the same, $R$-contraadjusted $r$-separated $r$-contramodule $R$-modules.

For any $R$-module $M$ and integer $n \geq 1$, any element $\bar{x} \in M / h_{r}(M)$ such that $r^{n} \bar{x}=0$ in $M / h_{r}(M)$ can be lifted to an element $x \in M$ such that $r^{n} x=0$ in $M$. Hence, the $r$-torsion in $M / h_{r}(M)$ is bounded whenever the $r$-torsion in $M$ is bounded, but the converse is not true. In fact, the $r$-torsion in $M / h_{r}(M)$ is bounded if and only if the $r$-torsion in $M$ is a sum of bounded $r$-torsion and $r$-divisible $r$-torsion.

To sum up, the condition that the $r$-torsion in $R$ is bounded in Corollary 5.16 can be replaced with the weaker condition that the $r$-torsion in $R / h_{r}(R)$ is bounded. In other words, $r$-divisible $r$-torsion in $R$ does not yet present a problem for Corollary 5.16, but any non$r$-divisible unbounded $r$-torsion does (cf. Proposition 5.21). Consequently, the same applies to Main Lemma 1.7 (cf. the proof in Sect. 7). So we can prove the conclusion of Main Lemma 1.7 with our methods assuming that the $r$-torsion in $R$ is a sum of bounded $r$-torsion and $r$-divisible $r$-torsion.

\subsection{Quotseparated $r$-contramodule $R$-modules}

The rest of Sect. 5 is devoted to the discussion of a partial extension of Corollary 5.16 to arbitrary commutative rings $R$ with an element $r \in R$.

Notice first of all that, dropping the contraadjustness requirements, a given $r$-contramodule $R$-module needs to be a quotient of an $r$-separated $r$-contramodule $R$-module for the conclusion Corollary 5.16 to have a chance to hold for it. This idea is captured in the following definition. 
Definition 5.18 Let $R$ be a commutative ring and $r \in R$ be an element. An $r$-contramodule $R$ module is called quotseparated if it is a quotient $R$-module of an $r$-separated $r$-contramodule $R$-module. We denote the full subcategory of quotseparated $r$-contramodule $R$-modules by $R-\bmod _{r \text {-ctra }}^{\text {qs }} \subset R-\bmod _{r \text {-ctra. }}$

\section{Lemma 5.19 Let $R$ be a commutative ring and $r \in R$ be an element. Then}

(a) the full subcategory $R$ - $\bmod _{r \text {-ctra }}^{\mathrm{qs}}$ is closed under subobjects, quotient objects, and infinite products in $R$ - $\bmod _{r \text {-ctra; }}$;

(b) any $r$-contramodule $R$-module is an extension of two quotseparated $r$-contramodule $R$-modules.

Proof Part (a): the full subcategory $R$ - $\bmod _{r \text {-ctra }}^{\mathrm{qs}} \subset R$ - $\bmod _{r \text {-ctra }}$ is closed under quotient objects by definition, and it is closed under subobjects and infinite products because the full subcategory of $r$-separated $r$-contramodules is closed under subobjects and infinite products in $R-\bmod _{r \text {-ctra }}$ Part (b): for any $r$-contramodule $R$-module $C$, one has $\Delta_{r}(C)=C$; hence, $C$ is the middle term of the short exact sequence of $r$-contramodule $R$-modules provided by Sublemma 4.6. Now $\Lambda_{r}(C)=\lim _{n \geq 1} C / r^{n} C$ is an $r$-separated $r$-contramodule, and $\lim _{n \geq 1}^{1} r^{n} C$ is a quotient $r$-contramodule $R$-module of an $r$-separated $r$-contramodule $R$ module $\prod_{n \geq 1} r^{n} C$.

The following theorem is a generalization of Theorem 5.9 to the unbounded torsion case. As in Sect. 5.3, we set $\Re=\lim _{n \geq 1} R / r^{n} R$.

Theorem 5.20 Let $R$ be a commutative ring and $r \in R$ be an element. Then the forgetful functor $\mathfrak{R}$-contra $\longrightarrow R$-mod induces an equivalence of abelian categories $\mathfrak{R}$-contra $\simeq$ $R-\bmod _{r \text {-ctra }}^{\mathrm{qs}}$.

Proof For any $r$-contramodule $R$-module $C$, consider the $R$-module $R[C]$ freely generated by the elements of $C$. By the adjunction property of the functor $\Delta_{r}$, the natural surjective $R$-module morphism $R[C] \longrightarrow C$ induces an $r$-contramodule $R$-module morphism $\Delta_{r}(R[C]) \longrightarrow C$. According to the discussion in [25, Example 3.6(3)], the forgetful functor identifies the category $\mathfrak{R}$-contra with the full subcategory in $R$ - $\bmod _{r \text {-ctra }}$ consisting of all the $r$-contramodule $R$-modules $C$ for which the morphism $\Delta_{r}(R[C]) \longrightarrow C$ factorizes as $\Delta_{r}(R[C]) \longrightarrow \Lambda_{r}(R[C]) \longrightarrow C$.

Now the $r$-contramodule $R$-module $\Lambda_{r}(R[C])$ is $r$-separated, and hence, the latter condition implies that the $r$-contramodule $R$-module $C$ is quotseparated. Conversely, if $C$ is the quotient of an $r$-separated $r$-contramodule $R$-module $B$, then the morphism $\Delta_{r}(R[C]) \longrightarrow$ $C$ factorizes through $B$, as $\Delta_{r}(R[C])$ is a projective object of $R$ - $\bmod _{r \text {-ctra }}$. Finally, for any $R$-module $A$, any morphism from $\Delta_{r}(A)$ to an $r$-separated $R$-module factorizes through $\Lambda_{r}(A)$, as $\Lambda_{r}(A)=\Lambda_{r}\left(\Delta_{r}(A)\right)$ is the maximal $r$-separated quotient $R$-module of $\Delta_{r}(A)$.

Let us point out that both the abelian categories $R-\bmod _{r \text {-ctra }}$ and $R-\bmod _{r \text {-ctra }}^{\mathrm{qs}}$ have enough projective objects [25,26], but the projective quotseparated $r$-contramodule $R$-modules are quite different from the projective $r$-contramodule $R$-modules.

The next proposition should be compared with the discussion in Remark 5.17.

Proposition 5.21 Let $R$ be a commutative ring and $r \in R$ be an element. Then all the $r$ contramodule $R$-modules are quotseparated if and only if the $r$-torsion in the ring $R / h_{r}(R)$ is bounded. 
Proof Notice that $\mathfrak{R}=\Lambda_{r}(R)=\Lambda_{r}\left(R / h_{r}(R)\right)$. So if the $r$-torsion in $R / h_{r}(R)$ is bounded, then $R-\bmod _{r \text {-ctra }}=R / h_{r}(R)-\bmod _{\bar{r} \text {-ctra }}=\Re$-contra by Theorem 5.9. Conversely, if $R$ - $\bmod _{r \text {-ctra }}=\mathfrak{R}$-contra, then the $r$-torsion in $\Delta_{r}(R)$ is bounded according to the discussion in [25, Example 5.2(7)], and $R / h_{r}(R)$ is a subring in $\Delta_{r}(R)$.

Remark 5.22 The following aspect of the notion of a quotseparated $r$-contramodule $R$-module is worth pointing out. All the conditions of $r$-contraadjustedness, $r$-contramoduleness, $r$ separatedness, and $r$-completeness of an $R$-module $C$ only depend on the underlying abelian group of $C$ and the additive operator $r: C \longrightarrow C$. So they can be equivalently considered as properties of modules over the ring of polynomials $\mathbb{Z}[r]$ rather than over a ring $R$.

On the other hand, all $r$-contramodule $\mathbb{Z}[r]$-modules are quotseparated by Proposition 5.21, but $r$-contramodule $R$-modules may be not. So an $r$-contramodule $R$-module $C$ always can be presented as a quotient $\mathbb{Z}[r]$-module of an $r$-separated $r$-contramodule $\mathbb{Z}[r]$-module $B$, but defining an action of the ring $R$ in $B$ compatible with the given action of $R$ in $C$ may be an impossible task when the ring $R$ contains too much $r$-torsion.

\subsection{Very flat cotorsion theory in quotseparated $r$-contramodule $\boldsymbol{R}$-modules}

The aim of this section is to prove the following generalization of Corollaries 5.15 and 5.16 to arbitrary commutative rings $R$ with an element $r \in R$. It claims that the quotseparatedness condition, which is necessary as we explained in the beginning of Sect. 5.5, is in fact also sufficient.

Proposition 5.23 Let $R$ be a commutative ring and $r \in R$ be an element. Then any $R$ contraadjusted quotseparated $r$-contramodule $R$-module can be presented as the cokernel of an injective morphism of $R$-contraadjusted $r$-separated $r$-complete $R$-modules.

The proof of Proposition 5.23 uses an approximation sequence in the very flat cotorsion theory on the category $R$ - $\bmod _{r \text {-ctra }}^{q s}=\mathfrak{R}$-contra, which is provided by the results of [22, Sections D.1 and D.4]. The exposition below in this section contains a sketch of some arguments from [22, Section D] specialized to the situation at hand.

Let $R$ be a commutative ring and $r \in R$ be an element. We keep the notation $\Re=$ $\lim _{n \geq 1} R / r^{n} R$. Recall from Sect. 5.3 that an $\Re$-contramodule $F$ is said to be flat if the $R / r^{n} R$ module $F / r^{n} F$ is flat for every $n \geq 1$. There are enough projective objects in $\Re$-contra, and all the projective $\Re$-contramodules are flat [22, Lemma D.1.6], [26, Lemma 6.9]. All the flat $\mathfrak{R}$-contramodules are $r$-separated [22, Corollary D.1.7], [26, Corollary 6.15]. The class of all flat $\mathfrak{R}$-contramodules is closed under extensions, the kernels of epimorphisms, and filtered inductive limits in $\Re$-contra [22, Lemmas D.1.4-5], [22, Corollary 7.1].

A quotseparated $r$-contramodule $R$-module $F$ is said to be very flat if the $R / r^{n} R$-module $F / r^{n} F$ is very flat for every $n \geq 1$ (cf. [22, Section C.3]) and Remark 5.14). This is equivalent to the $R / r^{n} R$-module $F / r^{n} F$ being flat for every $n \geq 1$ and the $R / r R$-module $F / r F$ being very flat [22, Lemma 1.6.8(b)]. All the projective quotseparated $r$-contramodule $R$-modules are very flat; the class of all very flat quotseparated $r$-contramodule $R$-modules is closed under extensions and the kernels of epimorphisms in $\mathfrak{R}-\bmod _{r \text {-ctra }}^{q \mathrm{~s}}$. The projective dimension of any very flat quotseparated $r$-contramodule $R$-module (as an object of $R$ - $\bmod _{r \text {-ctra }}^{\text {qs }}$ ) does not exceed 1 [22, Corollary D.4.1].

One can say that a quotseparated $r$-contramodule $R$-module $K$ is contraadjusted if $\operatorname{Ext}_{R \text {-mod }}^{1}$ qs $(F, K)=0$ for all very flat quotseparated $r$-contramodule $R$-modules $F$. Then the claim that the pair of full subcategories (very flat quotseparated $r$-contramodule $R$ modules, contraadjusted quotseparated $r$-contramodule $R$-modules) is a complete cotorsion 
theory in $R$ - $\bmod _{r \text {-ctra }}^{\text {qs }}$ follows already from the general results of [26, Example 7.12(3)]. The trick is to understand what the contraadjusted quotseparated $r$-contramodule $R$-modules are.

Recall that an $R / r R$-module is contraadjusted if and only if it is contraadjusted as an $R$-module [22, Lemma 1.6.6(a)] (cf. Lemma 7.3).

Lemma 5.24 Let $K$ be an $R$-module such that the $R / r R$-module $K / r K$ is contraadjusted. Then for any $n \geq 1$ the kernel of the natural surjective map $K / r^{n+1} K \longrightarrow K / r^{n} K$ is a contraadjusted $R / r R$-module.

Proof We have an exact sequence $K / r K \stackrel{r^{n}}{\longrightarrow} K / r^{n+1} K \longrightarrow K / r^{n} K \longrightarrow 0$, so the kernel of the map $K / r^{n+1} K \longrightarrow K / r^{n} K$ is a quotient $R / r R$-module of $K / r K$.

Let us introduce the following piece of terminology relevant for the argument in the rest of this section. We will say that an $R$-module $K$ is $r$-solid if it is $r$-separated, $r$-complete, and the $R / r R$-module $K / r K$ is contraadjusted. Obviously, all the $r$-solid $R$-modules belong to the category $R-\bmod _{r \text {-ctra }}^{\mathrm{qs}}$.

Lemma 5.25 Let $F$ be a flat $R$-module such that the $R / r R$-module $F / r F$ is very flat, and let $K$ be an $r$-solid $R$-module. Then $\operatorname{Ext}_{R}^{1}(F, K)=0$.

Proof Denoting the kernel of the morphism $K / r^{n+1} K \longrightarrow K / r^{n} K$ by $L_{n}, n \geq 0$, we have $\operatorname{Ext}_{R}^{1}\left(F, L_{n}\right)=\operatorname{Ext}_{R / r R}^{1}\left(F / r F, L_{n}\right)=0$ by Lemma 5.24. Hence, the assertion follows by virtue of the dual Eklof Lemma [6, Proposition 18].

Lemma 5.26 Let $F$ be a very flat quotseparated $r$-contramodule $R$-module and $K$ be an $r$-solid R-module. Then $\operatorname{Ext}_{R \text { - } \bmod _{r-c t r a}^{1}}^{q \text { s }}(F, K)=0$.

Proof This is [22, Lemma D.1.8]. Applying the Eklof Lemma for abelian categories [26, Lemma 4.5] to the category opposite to $R$ - $\bmod _{r \text {-ctra }}^{\mathrm{qs}}$ it remains to check that $\operatorname{Ext}_{R-\text { mod }_{r-\text { ctra }}^{1}}^{\text {qs }}(F, L)=0$ for any contraadjusted $R / r R$-module $L$. Let $0 \longrightarrow H \longrightarrow$ $G \longrightarrow \stackrel{r \text {-ctra }}{\longrightarrow} 0$ be a short exact sequence of quotseparated $r$-contramodule $R$-modules with a projective quotseparated $r$-contramodule $R$-module $G$. By [22, Lemma D.1.4] or [26, Lemma 6.7], the short sequence of $R / r R$-modules $0 \longrightarrow H / r H \longrightarrow G / r G \longrightarrow$ $F / r F \longrightarrow 0$ is exact. Since the $R / r R$-module $F / r F$ is very flat, it follows that any $R / r R$-module morphism $H / r H \longrightarrow L$ can be extended to an $R / r R$-module morphism $G / r G \longrightarrow L$. Thus any $R$-module morphism $H \longrightarrow L$ can be extended to an $R$-module morphism $G \longrightarrow L$.

Lemma 5.25 tells that the $r$-solid $R$-modules are contraadjusted as $R$-modules, while Lemma 5.26 shows they are contraadjusted as quotseparated $r$-contramodule $R$-modules (in the sense of the above definition). It follows, in particular, that the cokernel of any injective morphism of $r$-solid $R$-modules is also contraadjusted both in the sense of $R$-mod and $R-\bmod _{r \text {-ctra }}^{\text {qs }}(\mathrm{cf}$. Lemma 5.29).

The following construction plays a key role.

Lemma 5.27 Let $C$ be an $r$-separated $r$-contramodule $R$-module. Then there exists a short exact sequence of quotseparated $r$-contramodule $R$-modules $0 \longrightarrow C \longrightarrow K \longrightarrow F \longrightarrow 0$ with a very flat quotseparated $r$-contramodule $R$-module $F$ and an $r$-solid $R$-module $K$.

Proof This is a very simple particular case of [22, Lemma D.4.4]. Let $0 \longrightarrow C \longrightarrow K^{\prime} \longrightarrow$ $F^{\prime} \longrightarrow 0$ be a short exact sequence of $R$-modules with a very flat $R$-module $F^{\prime}$ and a 
contraadjusted $R$-module $K^{\prime}$. Then for every $n \geq 1$ the short sequence of $R / r^{n} R$-modules $0 \longrightarrow C / r^{n} C \longrightarrow K^{\prime} / r^{n} K^{\prime} \longrightarrow F^{\prime} / r^{n} F^{\prime} \longrightarrow 0$ is exact. Passing to the projective limit as $n \rightarrow \infty$, we obtain a short exact sequence $0 \longrightarrow C \longrightarrow K \longrightarrow F \longrightarrow 0$ with $C=\lim _{n} C / r^{n} C$ (by assumption), $K=\lim _{n} K^{\prime} / r^{n} K^{\prime}$ and $F=\lim _{n} F^{\prime} / r^{n} F^{\prime}$. As $K / r K={\overleftarrow{K^{\prime}}}^{n} r K^{\prime}$ is a contraadjusted $R / r R$-module and $F / r^{n} F=F^{\prime} / \overleftarrow{r}^{n} F^{\prime}$ are very flat $R / r^{n} R$-modules, all the desired properties hold.

Lemma 5.28 Any quotseparated $r$-contramodule $R$-module $C$ can be included into a short exact sequence of quotseparated $r$-contramodule $R$-modules $0 \longrightarrow K \longrightarrow F \longrightarrow C \longrightarrow 0$ with a very flat quotseparated $r$-contramodule $R$-module $F$ and an $r$-solid $R$-module $K$.

Proof Let $0 \longrightarrow B \longrightarrow P \longrightarrow C \longrightarrow 0$ be a short exact sequence of quotseparated $r$-contramodule $R$-modules with a projective quotseparated $r$-contramodule $R$-module $P$. Then the $R$-module $B$ is $r$-separated as a submodule of an $r$-separated $R$-module $P$, so the construction of Lemma 5.27 is applicable to $B$. It remains to use the construction from one of the Salce Lemmas [6, second paragraph of the proof of Theorem 10] in order to obtain the desired short exact sequence.

Lemma 5.29 Any quotseparated $r$-contramodule $R$-module $C$ can be included into a short exact sequence of quotseparated $r$-contramodule $R$-modules $0 \longrightarrow C \longrightarrow K \longrightarrow F \longrightarrow 0$ such that $F$ is a very flat quotseparated $r$-contramodule $R$-module and $K$ is the cokernel of an injective morphism of $r$-solid $R$-modules.

Proof Follows from Lemmas 5.28 and 5.27 (the latter of which has to be applied to a very flat quotseparated $r$-contramodule $R$-module-one needs to keep in mind that these are $r$-separated). We refer to [22, Corollary D.4.6] for the details.

Theorem 5.30 The pair of full subcategories (very flat quotseparated $r$-contramodule $R$-modules, $R$-contraadjusted quotseparated $r$-contramodule $R$-modules) is a hereditary complete cotorsion theory in $R$ - $\bmod _{r \text {-ctra }}^{q s}$. Moreover, a quotseparated $r$-contramodule $R$ module $K$ is $R$-contraadjusted if and only if the $R / r R$-module $K / r K$ is contraadjusted.

$I n$ other words, the three classes of quotseparated $r$-contramodule $R$-modules $K$ defined by the conditions

(i) $K$ is contraadjusted as a quotseparated $r$-contramodule $R$-module;

(ii) $K$ is contraadjusted as an $R$-module; and

(iii) the $R / r R$-module $K / r K$ is contraadjusted

coincide with each other.

Proof This is (an improvement over) [22, Corollary C.4.8]. The approximation sequences (with an $R$-contraadjusted quotseparated $r$-contramodule $R$-module $K$ ) are provided by Lemmas 5.28 and 5.29, so in order to prove all the assertions of the theorem it suffices to show that $\operatorname{Ext}_{R \text {-mod }}^{1}$ qs $(F, K)=0$ for any very flat quotseparated $r$-contramodule $R$ module $F$ and any quotseparated $r$-contramodule $R$-module $K$ such that the $R / r R$-module $K / r K$ is contraadjusted.

Indeed, applying Lemma 5.28 to the quotseparated $r$-contramodule $R$-module $K$, we obtain a short exact sequence of quotseparated $r$-contramodules $R$-modules $0 \longrightarrow L \longrightarrow$ $G \longrightarrow K \longrightarrow 0$ with an $r$-solid $R$-module $L$ and a very flat quotseparated $r$-contramodule $R$-module $G$. Tensoring with $R / r R$ over $R$, we get an exact sequence of $R / r R$-modules $L / r L \longrightarrow G / r G \longrightarrow K / r K \longrightarrow 0$. Since the class of contraadjusted $R / r R$-modules 
is closed under extensions and quotients, it follows that $G / r G$ is a contraadjusted $R / r R$ -

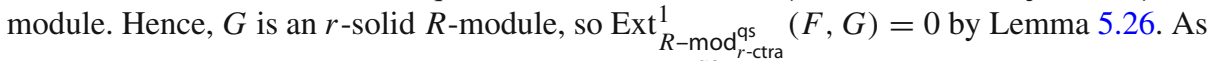
the projective dimension of the object $F \in R$ - $\bmod _{r \text {-ctra }}^{\text {qs }}$ does not exceed 1, it follows that $\operatorname{Ext}_{R-\bmod _{r-\text { ctra }}^{\text {qs }}}^{1}(F, K)=0$.

Proof of Proposition 5.23 Let $C$ be an $R$-contraadjusted quotseparated $r$-contramodule $R$ module. Applying Lemma 5.28, we obtain a short exact sequence $0 \longrightarrow K \longrightarrow F \longrightarrow$ $C \longrightarrow 0$ with an $r$-solid $R$-module $K$ and a very flat quotseparated $r$-contramodule $R$ module $F$. By Lemma 5.25, the $R$-module $K$ is contraadjusted, and it follows that so is the $R$-module $F$. As any flat quotseparated $r$-contramodule $R$-module, the $R$-module $F$ is $r$-separated. Thus $K \longrightarrow F$ is an injective morphism of $R$-contraadjusted $r$-separated $r$-contramodules with the cokernel $C$.

\section{Obtainable modules II}

In this section, as in Sect. 3 before, we work over an associative ring $R$.

Let $\mathrm{E}$ and $\mathrm{F}$ be two classes of left $R$-modules and $n \geq 1$ be an integer. Denote by ${ }^{\perp \geq n} \mathrm{E} \subset$ $R$-mod the class of all left $R$-modules such that $\operatorname{Ext}_{R}^{i}(F, E)=0$ for all $E \in \mathrm{E}$ and $i \geq n$, and by $\mathrm{F}^{\perp \geq n} \subset R$-mod the class of all left $R$-modules $C$ such that $\operatorname{Ext}_{R}^{i}(F, C)=0$ for all $F \in \mathrm{F}$ and $i \geq n$.

Let $\mathrm{E} \subset R$-mod be a fixed class of left $R$-modules. Set $\mathrm{F}={ }^{\perp_{\geq 1}} \mathrm{E}$ and $\mathrm{C}_{n}=\mathrm{F}^{\perp_{\geq n}}, n \geq 1$. Clearly, one has $C_{n} \subset C_{n+1}$ for all $n \geq 1$.

As in Sect. 3, our aim is to describe the class $C=C_{1}$ in terms of the class $E$. In this section, we develop a more powerful procedure of obtaining objects of the class $C_{1}$ from objects of the class $E$, which provides for the possibility of making detours through the classes $C_{n}$ (or, more specifically, $C_{2}$ ) when producing objects of the class $C_{1}$ from objects of the "seed" class E.

Lemma 6.1 For any class of left $R$-modules $F \subset R$-mod, the classes of left $R$-modules $C_{n}=F^{\perp \geq n}$ have the following properties:

(i) one has $C_{n} \subset C_{n+1}$ for all $n \geq 1$;

(ii) for every $n \geq 1$, the class $C_{n} \subset R$-mod is closed under the passages to direct summands, extensions, cokernels of injective morphisms, infinite products, and transfinitely iterated extensions in the sense of the projective limit;

(iii) the kernel of any surjective morphism from an object of $C_{n+1}$ to an object of $C_{n}$ belongs to $C_{n+1}$;

(iv) the cokernel of any injective morphism from an object of $C_{n+1}$ to an object of $C_{n}$ belongs to $C_{n}$.

Proof The property (i) holds by the definition, and (iii-iv) follow from the long exact sequence of $\operatorname{Ext}_{R}^{*}(F,-), \quad F \in \mathrm{F}$. To prove (ii), pick for every module $F \in \mathrm{F}$ one of its $(n-1)$-th syzygy modules, that is a module $G_{n-1}$ in an exact sequence $0 \longrightarrow G_{n-1} \longrightarrow P_{n-1} \longrightarrow$ $\cdots \longrightarrow P_{1} \longrightarrow F \longrightarrow 0$ with projective $R$-modules $P_{i}$. Denote by $\mathrm{G}_{n-1}, n \geq 2$ the class of all the left $R$-modules $G_{n-1}$ so obtained (and set $\mathrm{G}_{0}=\mathrm{F}$ ). Then one has $\mathrm{F}^{\perp \geq n}=\mathrm{G}_{n-1}^{\perp \geq 1}$ for every $n \geq 1$, and the assertion follows from Lemma 3.2 applied to the class $\mathrm{G}_{n-1} \subset R$-mod. 
Definition 6.2 The pair of classes of left $R$-modules right 1-obtainable and right 2-obtainable from a given class $\mathrm{E} \subset R$-mod is defined as the (obviously, unique) minimal pair of classes of left $R$-modules satisfying the following generation rules:

(i) all the $R$-modules from $\mathrm{E}$ are right 1-obtainable; all the right 1-obtainable $R$-modules are right 2-obtainable;

(ii) all the $R$-modules simply right obtainable (in the sense of Definition 3.3) from right 1obtainable $R$-modules are right 1-obtainable; all the $R$-modules simply right obtainable from right 2-obtainable $R$-modules are right 2-obtainable;

(iii) the kernel of any surjective morphism from a right 2-obtainable $R$-module to a right 1 -obtainable $R$-module is right 2-obtainable;

(iv) the cokernel of any injective morphism from a right 2-obtainable $R$-module to a right 1-obtainable $R$-module is right 1-obtainable.

The purpose of Definition 6.2 is that it describes a procedure according to which, starting from a seed class $E$, we first obtain some objects of the class $C_{1}$, then use them to produce some objects of the class $C_{2}$, and then use the latter in order to obtain even more objects of the class $C_{1}$, etc. A similar obtainability procedure involving $n$-obtainable modules for all $n \geq 2$ would be even more powerful, in the sense that it would possibly allow to obtain more modules (at least, in principle), but we will not use any $n$-obtainable modules beyond the 2-obtainable ones in the arguments in this paper, so we do not define them.

Lemma 6.3 For any class of left $R$-modules $\mathrm{E} \subset R$-mod, all the left $R$-modules right 1 obtainable from $\mathrm{E}$ belong to the class $\mathrm{C}_{1}=\left({ }^{\perp_{\geq 1}} \mathrm{E}\right)^{\perp_{\geq 1}} \subset R$-mod, and all the left $R$-modules right 2-obtainable from $\mathrm{E}$ belong to the class $\mathrm{C}_{2}=\left({ }^{\perp_{\geq 1}} \mathrm{E}\right)^{\perp_{\geq 2}} \subset R$-mod.

Proof Follows from Lemma 6.1.

Remark 6.4 As with the simple right obtainability rules of Definition 3.3, we will not use the full strength of Definition 6.2 in this paper. In addition to the limitations mentioned in Remark 3.7, the following one should be mentioned here: the second part of the rule (i) and the rule (iii) from Definition 6.2 will be only used in the following weak conjunction:

(iii') the kernel of any surjective morphism between two right 1-obtainable $R$-modules is right 2-obtainable.

Besides, not nearly all the complicated possibilities of transfinite iteration of the generation rules (i-iv) will be used in the actual constructions.

\section{Noetherian Main Lemma}

The proof of Main Lemmas 1.4 and 1.7 in this section is based on the results of Corollaries 5.15 and 5.16, respectively. The main lemmas are deduced from the following main propositions.

Main Proposition 7.1 Let $R$ be a Noetherian commutative ring and $r \in R$ be an element. Then an $R$-module is contraadjusted if and only if it is right 1-obtainable from contraadjusted $R / r R$-modules and contraadjusted $R\left[r^{-1}\right]$-modules.

Main Proposition 7.2 Let $R$ be a commutative ring and $r \in R$ be an element such that the $r$-torsion in $R$ is bounded. Then an $R$-module is contraadjusted if and only if it is right 1 -obtainable from contraadjusted $R / r R$-modules and contraadjusted $R\left[r^{-1}\right]$-modules. 
As the bounded torsion assumption in Corollary 5.16 can be weakened to the assumption that the $r$-torsion in $R$ is a sum of bounded $r$-torsion and $r$-divisible $r$-torsion (see Remark 5.17), this assumption in Main Proposition 7.2 and Main Lemma 1.7 also can be similarly weakened.

The following lemma shows that there is no ambiguity in the formulations of the main propositions.

Lemma 7.3 Let $f: R \longrightarrow R^{\prime}$ be a morphism of commutative rings such that for every element $r^{\prime} \in R^{\prime}$ there exist an element $r \in R$ and an invertible element $u \in R^{\prime}$ for which $r^{\prime}=u f(r)$. Then an $R^{\prime}$-module is contraadjusted if and only if it is contraadjusted as an $R$-module.

Proof By [22, Lemma 1.2.2(a)], contraadjustness is preserved by the restrictions of scalars, so all the contraadjusted $R^{\prime}$-modules are contraadjusted as $R$-modules. Conversely, let $C$ be an $R^{\prime}$-module that is contraadjusted as an $R$-module. We have to show that $\operatorname{Ext}_{R^{\prime}}^{1}\left(R^{\prime}\left[r^{\prime-1}\right], C\right)=$ 0 for all $r^{\prime} \in R^{\prime}$. By assumption, we have $r^{\prime}=u f(r)$, where $u \in R^{\prime}$ is invertible and $r \in R$. Hence, $R^{\prime}\left[r^{\prime-1}\right]=R^{\prime}\left[f(r)^{-1}\right]=R^{\prime} \otimes_{R} R\left[r^{-1}\right]$ and $\operatorname{Ext}_{R^{\prime}}^{1}\left(R^{\prime}\left[r^{\prime-1}\right], C\right)=$ $\operatorname{Ext}_{R}^{1}\left(R\left[r^{-1}\right], C\right)=0$.

In particular, an $R / r R$-module is contraadjusted if and only if it is contraadjusted as an $R$-module [22, Lemma 1.6.6(a)], and an $R\left[r^{-1}\right]$-module is contraadjusted if and only if it is contraadjusted as an $R$-module.

Lemma 7.4 Let $R$ be a commutative ring and $r \in R$ be an element. Then any $R$ contraadjusted $r$-separated $r$-complete $R$-module is an infinitely iterated extension, in the sense of the projective limit, of contraadjusted $R / r R$-modules.

Proof Let $K$ be an $R$-contraadjusted $r$-separated $r$-complete $R$-module. Then we have $K=$ $\lim _{n \geq 1} K / r^{n} K$, and it remains to apply Lemma 5.24.

Lemma 7.5 Let $R$ be a commutative ring and $r \in R$ be an element. Then the $R\left[r^{-1}\right]$-module $\operatorname{Hom}_{R}\left(R\left[r^{-1}\right], C\right)$ is contraadjusted for any contraadjusted $R$-module $C$.

Proof This is [22, Lemma 1.2.1(b) or Lemma 1.2.3(a)].

Lemma 7.6 For any commutative ring $R$, any $R$-module is right 2-obtainable from contraadjusted R-modules.

Proof Any $R$-module $A$ can be embedded into a contraadjusted (e.g., injective) $R$-module $B$. The quotient $R$-module $B / A$ is then also contraadjusted. Now $A$ is the kernel of the surjective morphism $B \longrightarrow B / A$, so it is right 2-obtainable from $B$ and $B / A$ according to the rules (i) and (iii) from Definition 6.2, or according to the rule (iii') from Remark 6.4.

Proof of Main Propositions 7.1 and 7.2 The (trivial) proof of the "if" assertion does not depend on the Noetherianity/bounded torsion assumption. Let $\mathrm{E}$ be a class of contraadjusted $R$ modules; then, all the very flat $R$-modules $F$ belong to the class $\mathrm{F}={ }^{\perp_{\geq 1}} \mathrm{E}$. By Lemma 6.3 , we have $C \in \mathrm{F}^{\perp \geq 1} \subset\{F\}^{\perp \geq 1}$ for all the $R$-modules $C$ right 1-obtainable from E; so all such $R$-modules $C$ are contraadjusted.

To prove the "only if" in either of the respective assumptions on the ring $R$ or the element $r$, denote by $\mathrm{E} \subset R$-mod the class of all contraadjusted $R / r R$-modules and contraadjusted $R\left[r^{-1}\right]$-modules (viewed as $R$-modules via the restriction of scalars). Let $C$ be a contraadjusted $R$-module. Our argument is based on the exact sequence (4). In view of the rules (ii) 
and (iv) from Definition 6.2, in order to show that $C$ is right 1-obtainable from $\mathrm{E}$ it suffices to check that the $R$-modules $\operatorname{Hom}_{R}\left(R\left[r^{-1}\right], C\right)$ and $\Delta_{r}(C)$ are right 1-obtainable from $\mathrm{E}$ and the $R$-module $\operatorname{Hom}_{R}\left(R\left[r^{-1}\right] / R, C\right)$ is right 2-obtainable from $\mathrm{E}$. Let us consider these three $R$-modules one by one.

The $R$-module $\operatorname{Hom}_{R}\left(R\left[r^{-1}\right], C\right)$ is a contraadjusted $R\left[r^{-1}\right]$-module by Lemma 7.5. So it already belongs to $\mathrm{E}$.

The $R$-module $\Delta_{r}(C)$ is contraadjusted as a quotient module of a contraadjusted $R$ module $C$. Besides, $\Delta_{r}(C)$ is an $r$-contramodule. Applying Corollary 5.15 or 5.16, we can present $\Delta_{r}(C)$ as the cokernel of an injective morphism of $R$-contraadjusted $r$-separated $r$-complete $R$-modules $K \longrightarrow L$. By Lemma 7.4, both $K$ and $L$ are obtainable as infinitely iterated extensions of contraadjusted $R / r R$-modules. Thus, the $R$-module $\Delta_{r}(C)$ is simply right obtainable from $\mathrm{E}$.

The $R$-module $\operatorname{Hom}_{R}\left(R\left[r^{-1}\right] / R, C\right)$ is an $r$-contramodule, and in fact even an $r$-separated $r$-complete $R$-module (see the proof of Toy Main Proposition 4.8). So it is simply right obtainable from $R / r R$-modules by Lemma 4.5 (Sublemma 4.7(a) is sufficient). By Lemma 7.6, all the $R / r R$-modules are right 2-obtainable from contraadjusted $R / r R$-modules. Hence, the $R$-module $\operatorname{Hom}_{R}\left(R\left[r^{-1}\right] / R, C\right)$ is right 2-obtainable from $\mathrm{E}$.

Proof of Main Lemmas 1.4 and 1.7 The "only if" assertion holds, because very flatness is preserved by the extensions of scalars (see Lemma 2.2(a)). The "if" assertion is the nontrivial part.

Let $F$ be an $R$-module such that the $R / r R$-module $F / r F$ is very flat and the $R\left[r^{-1}\right]$ module $F\left[r^{-1}\right]$ is very flat. In order to show that the $R$-module $F$ is very flat, we will check that $\operatorname{Ext}_{R}^{1}(F, C)=0$ for every contraadjusted $R$-module $C$.

As above, we denote by $\mathrm{E} \subset R$-mod the class of all contraadjusted $R / r R$-modules and contraadjusted $R\left[r^{-1}\right]$-modules. Let us check that $\operatorname{Ext}_{R}^{i}(F, E)=0$ for all $E \in \mathrm{E}$ and $i \geq 1$. Indeed, if $E$ is a contraadjusted $R\left[r^{-1}\right]$-module, then we have $\operatorname{Ext}_{R}^{i}(F, E)=$ $\operatorname{Ext}_{R\left[r^{-1}\right]}^{i}\left(F\left[r^{-1}\right], E\right)=0$ by Lemma 4.1(a), and if $E$ is a contraadjusted $R / r R$-module, we have $\operatorname{Ext}_{R}^{i}(F, E)=\operatorname{Ext}_{R / r R}^{i}(F / r F, E)=0$, also by Lemma 4.1(a). Hence, we have $F \in \mathrm{F}={ }^{\perp} \geq 1 \mathrm{E}$.

According to Main Proposition 7.1 or 7.2, the $R$-module $C$ is right 1-obtainable from $\mathrm{E}$. By Lemma 6.3, it follows that $C \in \mathrm{F}^{\perp \geq 1}$, $\operatorname{so}_{\operatorname{Ext}_{R}^{1}}^{1}(F, C)=0$.

The proof of Main Theorem 1.3 is now finished.

\section{Finitely very flat Main Lemma}

Let us recall the setting of Sects. 1.8-1.9. We consider an arbitrary commutative ring $R$ and a finite set of elements $\mathbf{r}=\left\{r_{1}, \ldots, r_{m}\right\} \subset R$. For every subset $J \subset\{1, \ldots, m\}$, we denote by $r_{J}$ the product $\prod_{j \in J} r_{j} \in R$. The set of all elements $\left\{r_{J}\right\}$ is denoted by $\mathbf{r}^{\times}$. We put $K=\{1, \ldots, m\} \backslash J$, and denote by $R_{J}$ the localization-quotient ring $\left(R / \sum_{k \in K} r_{k} R\right)\left[r_{J}^{-1}\right]$ obtained by annihilating all the elements $r_{k}, k \in K$, and inverting all the elements $r_{j}, j \in J$ in the ring $R$.

An $R$-module $C$ is said to be $\mathbf{r}$-contraadjusted if $\operatorname{Ext}_{R}^{1}\left(R\left[r_{j}^{-1}\right], C\right)=0$ for all $1 \leq j \leq m$, and an $R$-module $F$ is said to be $\mathbf{r}$-very flat if $\operatorname{Ext}_{R}^{1}(F, C)=0$ for every $\mathbf{r}$-contraadjusted $R$-module $C$ (see Sect. 1.3 for a discussion). We are mostly interested in $\mathbf{r}^{\times}$-contraadjusted and $\mathbf{r}^{\times}$-very flat $R$-modules. 
In this section, we start with proving Theorem 1.9 and then proceed to deduce Main Lemma 1.6.

Main Proposition 8.1 Let $R$ be a commutative ring and $r_{1}, \ldots, r_{m} \in R$ be a finite collection of its elements. Then an $R$-module is $\mathbf{r}^{\times}$-contraadjusted if and only if it is right 1-obtainable from $R_{J}$-modules, where $J \subset\{1, \ldots, m\}$.

Notice first of all that it follows from Main Proposition 8.1 (by means of Lemma 7.6) that all $R$-modules are right 2-obtainable from $R_{J}$-modules.

Lemma 8.2 Let $R$ be a commutative ring and $r_{1}, \ldots, r_{m} \in R$ be a finite set of elements. Let $C$ be an $R$-module such that $C$ is an $r_{k}$-contramodule for every $1 \leq k \leq m$. Then $C$ is simply right obtainable from $R /\left(r_{1} R+\cdots+r_{m} R\right)$-modules (viewed as $R$-modules via the restriction of scalars).

Proof This is a generalization of Lemma 4.5 to the case of a finite set of elements in $R$ instead of just one element. One can prove it in a way similar to the proof of Lemma 4.5. Specifically, let $I \subset R$ denote the ideal generated by the elements $r_{1}, \ldots, r_{m}$. According to [24, Theorem 5.1], the full subcategory of all $R$-modules that are $r_{k}$-contramodules for all $1 \leq k \leq m$ depends only on the ideal $I$ (and, in fact, even only on its radical $\sqrt{I} \subset R$ ) rather than on a specific set of its generators $r_{k}$. Such $R$-modules are called $I$-contramodules, and the full subcategory formed by them is denoted by $R-\bmod _{I \text {-ctra }} \subset R$-mod. The embedding functor $R-\bmod _{I \text {-ctra }} \longrightarrow R-\bmod$ has a left adjoint, which is denoted by $\Delta_{I}: R-\bmod \longrightarrow$ $R-\bmod _{I \text {-ctra }}[24$, Theorem 7.2].

For every $n \geq 1$, denote by $I_{n} \subset R$ the ideal generated by the elements $r_{1}^{n}, \ldots, r_{m}^{n} \in R$. So one has $I_{n} \subset I^{n} \subset R$, and for any integer $n \geq 1$ there exists an integer $N>n$ such that $I^{N} \subset I_{n}$.

The following two sublemmas allow to deduce Lemma 8.2 in the same way as Lemma 4.5 is deduced from Sublemmas 4.6 and 4.7.

Sublemma 8.3 Let $R$ be a commutative ring, $r_{1}, \ldots, r_{m} \in R$ be a finite set of its elements, and $I=\left(r_{1}, \ldots, r_{m}\right) \subset R$ be the ideal generated by them. Then for any $R$-module $A$ there is a natural short exact sequence of $R$-modules

$$
0 \longrightarrow \lim _{n \geq 1}^{1} D_{n}(A) \longrightarrow \Delta_{I}(A) \longrightarrow \lim _{n \geq 1} A / I^{n} A \longrightarrow 0
$$

where $D_{n}(A)$ are certain $R / I_{n} R$-modules forming a projective system indexed by the positive integers (which depends functorially on $A$ ).

Proof This is [24, Lemma 7.5].

Sublemma 8.4 Let $R$ be a commutative ring and $r_{1}, \ldots, r_{m} \in R$ be a finite set of its elements. Let $D_{1} \longleftarrow D_{2} \longleftarrow D_{3} \longleftarrow \cdots$ be a projective system of $R$-modules such that $D_{n}$ is an $R / I_{n} R$-module for every $n \geq 1$. Then the $R$-modules (a) $\lim _{n} D_{n}$ and (b) $\lim _{n}^{1} D_{n}$ are simply right obtainable from $R /\left(r_{1} R+\cdots+r_{m} R\right)$-modules.

Proof Similar to the proof of Sublemma 4.7 (cf. [24, proof of Theorem 9.5]).

Alternatively, one can deduce Lemma 8.2 from (the proof of) Lemma 4.5 arguing by induction in the cardinality $m$ of the set of elements $\left\{r_{k}\right\}$. Inspecting the proof of Lemma 4.5, one can observe that all the $R$-modules involved in obtaining an $r$-contramodule $R$-module $C$ from $R / r R$-modules according to this proof depend functorially on $C$. Moreover, these 
functors are nothing but various compositions of the basic operations of the passage to the kernel, cokernel, and infinite product.

For any second element $s \in R$, these operations preserve the full subcategory of $s$ contramodule $R$-modules $R$ - $\bmod _{s \text {-ctra }} \subset R$-mod. Thus, given an $r$-contramodule and $s$ contramodule $R$-module $C$, the constructions of Lemma 4.5 show that $C$ is simply right obtainable from $R / r R$-modules that are also $s$-contramodules.

Similarly, given an $R$-module $C$ that is an $r_{k}$-contramodule for every $1 \leq k \leq m$, the constructions of Lemma 4.5 show that $C$ is simply right obtainable from $R / r_{m} R$-modules that are $r_{k}$-contramodules for every $1 \leq k \leq m$. Passing to the ring $R / r_{m} R$ and proceeding by induction in $m$, one proves that $C$ is simply right obtainable from $R /\left(r_{1} R+\cdots+r_{m} R\right)$ modules.

Lemma 8.5 Let $R$ be a commutative ring and $r \in R$ be an element. Let $L^{\bullet} \in \mathrm{D}^{\mathrm{b}}(R-\mathrm{mod})$ be a bounded complex of $R$-modules and $C$ be an $r$-contramodule $R$-module. Then the $R$-modules $\operatorname{Hom}_{\mathrm{D}(R \text {-mod })}\left(L^{\bullet}, C[i]\right), i \in \mathbb{Z}$ are also r-contramodules.

Proof This is [24, Lemma 6.2(b)].

Lemma 8.6 Let $R$ be a commutative ring and $r, s \in R$ be two elements. Assume that an $R$ module $C$ is ( $r$ s)-contraadjusted. Then the $R$-module $\operatorname{Hom}_{k}\left(R\left[r^{-1}\right], C\right)$ is s-contraadjusted.

Proof For any three $R$-modules $F, G$, and $C$, there is a pair of spectral sequences

$$
\begin{aligned}
{ }^{\prime} E_{2}^{p q} & =\operatorname{Ext}_{R}^{p}\left(G, \operatorname{Ext}_{R}^{q}(F, C)\right) \Longrightarrow H^{p+q}\left(\mathbb{R} \operatorname{Hom}_{R}\left(G, \mathbb{R} \operatorname{Hom}_{R}(F, C)\right)\right) \\
{ }^{\prime \prime} E_{2}^{p q} & =\operatorname{Ext}_{R}^{p}\left(\operatorname{Tor}_{q}^{R}(F, G), C\right) \Longrightarrow H^{p+q}\left(\mathbb{R} \operatorname{Hom}_{R}\left(F \otimes_{R}^{\mathbb{L}} G, C\right)\right)
\end{aligned}
$$

with the differentials $d_{r}^{p q}: E_{2}^{p q} \longrightarrow E_{2}^{p+r, q-r+1}$, converging to the same limit $E_{\infty}^{p+q}=$ $H^{p+q}\left(\mathbb{R} \operatorname{Hom}_{R}\left(G, \mathbb{R} \operatorname{Hom}_{R}(F, C)\right)\right)=H^{p+q}\left(\mathbb{R} \operatorname{Hom}_{R}\left(F \otimes_{R}^{\mathbb{L}} G, C\right)\right)$. In low degrees, each of them turns into an exact sequence

$$
0 \longrightarrow E_{2}^{1,0} \longrightarrow E_{\infty}^{1} \longrightarrow E_{2}^{0,1} \longrightarrow E_{2}^{2,0} \longrightarrow E_{\infty}^{2} .
$$

In particular, if $\operatorname{Tor}_{1}^{R}(F, G)=0$, then ${ }^{\prime \prime} E_{2}^{0,1}=0$ implies ${ }^{\prime \prime} E_{2}^{1,0}=E_{\infty}^{1}$, leading to an injective morphism

$$
\operatorname{Ext}_{R}^{1}\left(F, \operatorname{Hom}_{R}(G, C)\right)={ }^{\prime} E_{2}^{1,0} \longrightarrow E_{\infty}^{1}={ }^{\prime \prime} E_{2}^{1,0}=\operatorname{Ext}_{R}^{1}\left(F \otimes_{R} G, C\right) .
$$

Thus, in particular, for any $R$-module $C$, there is a natural injective $R$-module morphism

$$
\operatorname{Ext}_{R}^{1}\left(R\left[s^{-1}\right], \operatorname{Hom}_{R}\left(R\left[r^{-1}\right], C\right)\right) \longrightarrow \operatorname{Ext}_{R}^{1}\left(R\left[(r s)^{-1}\right], C\right),
$$

so the left-hand side vanishes whenever the right-hand side does (cf. [22, proof of Lemma 1.2.1]).

Proof of Main Proposition 8.1 Denote by $\mathrm{E} \subset R$-mod the class of all $R_{J}$-modules, where $J$ runs over the subsets of $\{1, \ldots, m\}$, viewed as $R$-modules via the restriction of scalars. We have to show that an $R$-module $C$ is $\mathbf{r}^{\times}$-contraadjusted if and only if it is right 1-obtainable from $\mathrm{E}$.

To prove the "if", notice that all the $R$-modules from the class $\mathrm{E}$ are $\mathbf{r}^{\times}$-contraadjusted. Indeed, let $I$ and $J$ be two subsets in $\{1, \ldots, m\}$, and $E$ be an $R_{J}$-module. Then we have

$$
R_{J} \otimes_{R} R\left[r_{I}^{-1}\right]= \begin{cases}R_{J}, & \text { if } I \subset J, \\ 0, & \text { if } I \not \subset J .\end{cases}
$$


By Lemma 4.1(a), it follows that $\operatorname{Ext}_{R}^{i}\left(R\left[r_{I}^{-1}\right], E\right)=\operatorname{Ext}_{R_{J}}^{i}\left(R_{J} \otimes_{R} R\left[r_{I}^{-1}\right], E\right)=0$ for $i \geq 1$ in both cases.

In other words, we have $R\left[r_{I}^{-1}\right] \in \mathrm{F}={ }^{\perp_{\geq 1}} \mathrm{E}$ for all $I \subset\{1, \ldots, m\}$. By Lemma 6.3, it follows that $C \in \mathrm{F}^{\perp_{\geq 1}} \subset\left\{R\left[r_{I}^{-1}\right]\right\}^{\perp_{\geq 1}}$ for every $R$-module $C$ right 1-obtainable from $\mathrm{E}$, so $\operatorname{Ext}_{R}^{1}\left(R\left[r_{I}^{-1}\right], C\right)=0$ and $C$ is $\mathbf{r}^{\times}$-contraadjusted.

The nontrivial part is the "only if." Before spelling out formally the induction procedure constituting our argument, let explain the key ideas first in some informal terms. What we are doing here can be described as "cutting modules into pieces." The main proposition claims that any $\mathbf{r}^{\times}$-contraadjusted $R$-module can be cut into certain kind of pieces of simple nature.

We have a finite collection of elements $r_{1}, \ldots, r_{m}$, and so we work with them one by one. Using the exact sequence (4), an $r_{m}$-contraadjusted $R$-module $C$ can be cut into three pieces of two sorts (meaning that we need to show two of them to be 1-obtainable from $\mathrm{E}$, while the third one can be only 2-obtainable). One of these three pieces is an $R\left[r_{m}^{-1}\right]$-module, while the other two are $r_{m}$-contramodule $R$-modules.

As with any such inductive transformation procedure, the trick is to make sure that we do not lose important properties along the way. The original module $C$ is not just $r_{m}$-contraadjusted; it is $\mathbf{r}^{\times}$-contraadjusted. The pieces into which we cut it should remain $\mathbf{r}^{\times}$-contraadjusted, or something close to that; or otherwise our induction procedure would not work. To be more precise, when we get a piece about which we only need to show that it is 2-obtainable, we are not concerned with its contraadjustness properties anymore; but to show that a module is 1-obtainable, we need to know that it is $r_{J}$-contraadjusted for various elements $r_{J} \in \mathbf{r}^{\times}$.

The problem is that contraadjustness is a fragile property. It is destroyed by passages to the kernels of morphisms. Transforming modules using the exact sequence (4) does not involve passages to the kernels, but the constructions of Sublemmas 4.6-4.7 or 8.3-8.4 do. So we cannot just cut an $r_{m}$-contramodule $R$-module into $R / r_{m} R$-modules, using constructions of this kind, without possibly destroying our contraadjustness properties with respect to the other elements $r_{j}$ or $r_{J}$.

The solution implemented in the argument below is to postpone all the cutting of $r_{j}$ contramodules into $R / r_{j} R$-modules until the moment after we have already applied the exact sequence (4) along all the elements $r=r_{j}, j=1, \ldots, m$, replacing all the contraadjustness with either invertibility of $r_{j}$, or $r_{j}$-contramoduleness, for every $1 \leq j \leq m$ (or rather, strengthening all the contraadjustness to various combinations of invertibility and contramoduleness). Invertibility and contramoduleness are robust properties, preserved by passages to the kernels, cokernels, and infinite products, so applying the constructions of Sublemmas $8.3-8.4$ becomes possible at this point and no vital information is destroyed.

Here is the promised formal induction procedure. We will prove the following assertion by induction on $0 \leq n \leq m$ : any $R$-module that is $r_{J}$-contraadjusted for all $J \subset\{1, \ldots, n\}$ and an $r_{k}$-contramodule for all $n+1 \leq k \leq m$, is right 1-obtainable from $R_{J}$-modules, where $J \subset\{1, \ldots, n\} \subset\{1, \ldots, m\}$. For $n=m$, this is the assertion of the main proposition.

Induction base: for $n=0$, this is the assertion of Lemma 8.2.

Let $C$ be an $R$-module that is $r_{J}$-contraadjusted for all $J \subset\{1, \ldots, n\}$ and an $r_{k^{-}}$ contramodule for all $n+1 \leq k \leq m$. Consider the exact sequence (4) for $r=r_{n}$ :

$$
0 \longrightarrow \operatorname{Hom}_{R}\left(R\left[r_{n}^{-1}\right] / R, C\right) \longrightarrow \operatorname{Hom}_{R}\left(R\left[r_{n}^{-1}\right], C\right) \longrightarrow C \longrightarrow \Delta_{r_{n}}(C) \longrightarrow 0 \text {. }
$$

Denote by $\mathrm{E}_{n} \subset R$-mod the class of all $R_{J}$-modules with $J \subset\{1, \ldots, n\}$. To show that $C$ is right 1-obtainable from $\mathrm{E}_{n}$, it suffices to check that the $R$-modules $\operatorname{Hom}_{R}\left(R\left[r_{n}^{-1}\right], C\right)$ 
and $\Delta_{r_{n}}(C)$ are right 1-obtainable from $\mathrm{E}_{n}$ and the $R$-module $\operatorname{Hom}_{R}\left(R\left[r_{n}^{-1}\right] / R, C\right)$ is right 2-obtainable from $\mathrm{E}_{n}$. Let us consider these three $R$-modules one by one.

The $R$-module $\operatorname{Hom}_{R}\left(R\left[r_{n}^{-1}\right], C\right)$ is an $R\left[r_{n}^{-1}\right]$-module. By Lemma 8.5 , it is also an $r_{k}$-contramodule for all $n+1 \leq k \leq m$, and by Lemma 8.6, it is $r_{J}$-contraadjusted for all $J \subset\{1, \ldots, n-1\}$. In order to conclude that the $R$-module $\operatorname{Hom}_{R}\left(R\left[r_{n}^{-1}\right], C\right)$ is right 1-obtainable from $\mathrm{E}_{n}$, it remains to apply the induction assumption to the pair of integers $\left(n^{\prime}, m^{\prime}\right)=(n-1, m-1)$, the ring $R^{\prime}=R\left[r_{n}^{-1}\right]$, and the set of elements $r_{1}^{\prime}, \ldots, r_{m-1}^{\prime} \in R^{\prime}$ equal to the images of the elements $r_{1}, \ldots, r_{n-1}, r_{n+1}, \ldots, r_{m}$ under the localization morphism $R \longrightarrow R\left[r_{n}^{-1}\right]$.

The $R$-module $\Delta_{r_{n}}(C)$ is $r_{J}$-contraadjusted for all $J \subset\{1, \ldots, n-1\}$ as a quotient module of an $r_{J}$-contraadjusted $R$-module $C$. It is also an $r_{n}$-contramodule (because the $R$ module $\Delta_{r}(A)$ is an $r$-contramodule for any $R$-module $A$ ), and it is an $r_{k}$-contramodule for all $n+1 \leq k \leq m$ by Lemma 8.5. So $\Delta_{r_{n}}(C)$ is an $r_{k}$-contramodule for all $n \leq k \leq m$. The claim that the $R$-module $\Delta_{r_{n}}(C)$ is right 1-obtainable from $\mathrm{E}_{n}$ (in fact, even from $\mathrm{E}_{n-1}$ ) now follows from the induction assumption applied to the pair of integers $\left(n^{\prime}, m^{\prime}\right)=(n-1, m)$, the same ring $R^{\prime}=R$, and the same set of elements $r_{1}^{\prime}=r_{1}, \ldots, r_{m}^{\prime}=r_{m}$.

The $R$-module $\operatorname{Hom}_{R}\left(R\left[r_{n}^{-1}\right] / R, C\right)$ is an $r_{n}$-contramodule (in fact, even an $r_{n}$-separated $r_{n}$-complete $R$-module) and an $r_{k}$-contramodule for all $n+1 \leq k \leq m$ (by Lemma 8.5). By Lemma 8.2, the $R$-module $\operatorname{Hom}_{R}\left(R\left[r_{n}^{-1}\right] / R, C\right)$ is simply right obtainable from $R /\left(r_{n} R+\right.$ $\cdots+r_{m} R$ )-modules.

Now we apply the induction assumption to the pair of integers $\left(n^{\prime}, m^{\prime}\right)=(n-1, n-1)$, the ring $R^{\prime}=R /\left(r_{n} R+\cdots+r_{m} R\right)$, and the sequence of elements $r_{1}^{\prime}, \ldots, r_{n-1}^{\prime} \in R^{\prime}$ equal to the images of the elements $r_{1}, \ldots, r_{n-1}$ under the surjective ring homomorphism $R \longrightarrow R^{\prime}$. This is simply the assertion of the main proposition for the ring $R^{\prime}$ with $n-1$ elements $r_{1}^{\prime}, \ldots, r_{n-1}^{\prime}$. By Lemma 7.6, this assertion implies that every $R^{\prime}$-module is right 2-obtainable from $\mathrm{E}_{n-1}$. Thus, the $R$-module $\operatorname{Hom}_{R}\left(R\left[r_{n}^{-1}\right] / R, C\right)$ is right 2-obtainable from $\mathrm{E}_{n-1}$.

Proof of Theorem 1.9 As in the proof of Toy Main Lemma 1.8 in Sect. 4, the "only if" assertion holds, because the functors $F \longmapsto R_{J} \otimes_{R} F, J \subset\{1, \ldots, m\}$ preserve transfinitely iterated extensions, in the sense of the inductive limit, of flat $R$-modules, and take the $R$-modules $R\left[r_{I}^{-1}\right], I \subset\{1, \ldots, m\}$ to free $R_{J}$-modules (with 1 or 0 generators). The "if" assertion is the nontrivial part.

To show that the $R$-module $F$ is $\mathbf{r}^{\times}$-very flat, we need to check that $\operatorname{Ext}_{R}^{1}(F, C)=0$ for all $\mathbf{r}^{\times}$-contraadjusted $R$-modules $C$. As in the proof of Main Proposition 8.1, we denote by $\mathrm{E} \subset R$-mod the class of all $R_{J}$-modules, $J \subset\{1, \ldots, m\}$.

By Lemma 4.1(a), it follows from the assumption of flatness of the $R$-module $F$ and projectivity of the $R_{J}$-modules $R_{J} \otimes_{R} F$ that $\operatorname{Ext}_{R}^{i}(F, E)=0$ for all $E \in \mathrm{E}$ and $i \geq 1$. So $F \in{ }^{\perp_{\geq 1}} \mathrm{E}$. By Main Proposition 8.1, the $R$-module $C$ is right 1-obtainable from E. Applying Lemma 6.3, we can conclude that $C \in\left({ }^{\perp_{\geq 1}} \mathrm{E}\right)^{\perp_{\geq 1}} \subset\{F\}^{\perp_{\geq 1}}$, and hence, $\operatorname{Ext}_{R}^{1}(F, C)=0$.

Proof of Main Lemma 1.6 The "only if" assertion holds, because finite very flatness is preserved by the extensions of scalars (see Lemma 2.2(b)). The "if" assertion is the nontrivial part.

Let us recall the discussion from Sect. 1.8, with a slight change in notation. We have a finite set of elements $\mathbf{S}=\left\{s_{1}, \ldots, s_{p}\right\}$ in the quotient ring $R / r R$ such that the $R / r R$-module $F / r F$ is $\mathbf{s}^{\times}$-very flat, and a finite set $\mathbf{t}=\left\{t_{1}, \ldots, t_{q}\right\}$ of elements in the ring of fractions $R\left[r^{-1}\right]$ such that the $R\left[r^{-1}\right]$-module $F\left[r^{-1}\right]$ is $\mathbf{t}^{\times}$-very flat. Lift the elements $s_{i} \in R / r R$ to some elements $\tilde{s}_{i} \in R$, and choose elements $\tilde{t}_{l} \in R$ such that $t_{l}=\tilde{t}_{l} / r^{n_{l}}$ in $R\left[r^{-1}\right]$ for some exponents $n_{l} \geq 0$. 
Set $r_{0}=r, r_{i}=\tilde{s}_{i}$ for $1 \leq i \leq p$, and $r_{p+l}=\tilde{t}_{l}$ for $1 \leq l \leq q$. Denote the set of all elements $r_{0}, \ldots, r_{p+q} \in R$ by $\mathbf{r}$. We claim that the $R$-module $F$ is $\mathbf{r}^{\times}$-very flat.

According to Theorem 1.9, it suffices to check that the $R_{J}$-module $R_{J} \otimes_{R} F$ is projective for all subsets $J \subset\{0, \ldots, p+q\}$. Let us consider two cases separately.

If $0 \notin J$, then the ring homomorphism $R \longrightarrow R_{J}$ factorizes as $R \longrightarrow R / r R \longrightarrow R_{J}$. Denote by $J^{\prime}$ the set $J \cap\{1, \ldots, p\}$. Then, moreover, the ring homomorphism $R / r R \longrightarrow R_{J}$ factorizes as $R / r R \longrightarrow(R / r R)_{J^{\prime}} \longrightarrow R_{J}$, where the notation is $K^{\prime}=\{1, \ldots, p\} \backslash J^{\prime}$, $s_{J^{\prime}}=\prod_{j \in J^{\prime}} s_{j}$, and $(R / r R)_{J^{\prime}}=\left((R / r R) / \sum_{k \in K^{\prime}} s_{k}(R / r R)\right)\left[s_{J^{\prime}}^{-1}\right]$.

Since the $R / r R$-module $F / r F$ is $\mathbf{s}^{\times}$-very flat, by the "only if" assertion of Theorem 1.9 the $(R / r R)_{J^{\prime}}$-module $(R / r R)_{J^{\prime}} \otimes_{R} F=(R / r R)_{J^{\prime}} \otimes_{R / r R} F / r F$ is projective, and it follows that the $R_{J}$-module $R_{J} \otimes_{R} F$ is also projective.

If $0 \in J$, then the ring homomorphism $R \longrightarrow R_{J}$ factorizes as $R \longrightarrow R\left[r^{-1}\right] \longrightarrow R_{J}$. Denote by $J^{\prime \prime} \subset\{1, \ldots, q\}$ the set of all integers $j-p$, where $j \in J \cap\{p+1, \ldots, p+$ $q$ \}. Then, moreover, the ring homomorphism $R\left[r^{-1}\right] \longrightarrow R_{J}$ factorizes as $R\left[r^{-1}\right] \longrightarrow$ $R\left[r^{-1}\right]_{J^{\prime \prime}} \longrightarrow R_{J}$, where the notation is $K^{\prime \prime}=\{1, \ldots, q\} \backslash J^{\prime \prime}, \quad t_{J^{\prime \prime}}=\prod_{j \in J^{\prime \prime}} t_{j}$, and $R\left[r^{-1}\right]_{J^{\prime \prime}}=\left(R\left[r^{-1}\right] / \sum_{k \in K^{\prime \prime}} t_{k} R\left[r^{-1}\right]\right)\left[t_{J^{\prime \prime}}^{-1}\right]$.

Since the $R\left[r^{-1}\right]$-module $F\left[r^{-1}\right]$ is $\mathbf{t}^{\times}$-very flat, by the "only if" assertion of Theorem 1.9 the $R\left[r^{-1}\right]$-module $R\left[r^{-1}\right]_{J^{\prime \prime}} \otimes_{R} F=R\left[r^{-1}\right]_{J^{\prime \prime}} \otimes_{R\left[r^{-1}\right]} F\left[r^{-1}\right]$ is projective, and it follows that the $R_{J}$-module $R_{J} \otimes_{R} F$ is also projective.

This finishes the proof of Main Theorem 1.5, and consequently also of Main Theorems 1.2 and 1.1.

\section{Examples and applications}

Let $R$ be a commutative ring. An $R$-algebra is said to be flat if it is a flat $R$-module. A commutative $R$-algebra $S$ is called very flat if the $R$-module $S\left[s^{-1}\right]$ is very flat for every element $s \in S$ (see the discussion in Sect. 0.6).

Corollary 9.1 Let $R$ be a commutative ring. Then any finitely presented, flat $R$-algebra is very flat.

Proof Let $S$ be a finitely presented flat $R$-algebra and $s \in S$ be an element. Then the $R$-module $S\left[s^{-1}\right]$ is flat, because it is a directed inductive limit of copies of the free $S$-module $S$, which is a flat $R$-module by assumption. Furthermore, the $R$-algebra $S\left[s^{-1}\right]$ is finitely presented, since the $R$-algebra $S$ is. Indeed, if $S$ is the quotient algebra of the algebra of polynomials $R\left[x_{1}, \ldots, x_{m}\right]$ by a finitely generated ideal $I$, then $S\left[s^{-1}\right]$ is the quotient algebra of $R\left[x_{1}, \ldots, x_{m}, z\right]$ by the finitely generated ideal $J$ whose generators are the generators of the ideal $I$ and the element $z q\left(x_{1}, \ldots, x_{m}\right)-1$, where $q \in R\left[x_{1}, \ldots, x_{m}\right]$ is a preimage of the element $s \in S$. Applying Main Theorem 1.1, we conclude that $S\left[s^{-1}\right]$ is a very flat $R$-module.

Let $R$ be a commutative ring. A commutative $R$-algebra $S$ is said to be finitely very flat if the $R$-module $S\left[s^{-1}\right]$ is finitely very flat for every element $s \in S$. The following corollary is a stronger version of Corollary 9.1 .

Corollary 9.2 Let $R$ be a commutative ring. Then any finitely presented, flat $R$-algebra is finitely very flat. 
Proof Let $S$ be a finitely presented flat $R$-algebra and $s \in S$ be an element. As it was explained in the proof of Corollary 9.1, $S\left[s^{-1}\right]$ is a finitely presented flat $R$-algebra, too. Applying Main Theorem 1.5 to the $R$-algebra $S\left[s^{-1}\right]$ and the $S\left[s^{-1}\right]$-module $F=S\left[s^{-1}\right]$, we conclude that the $R$-module $S\left[s^{-1}\right]$ is finitely very flat.

The next lemma explains the importance of (finitely) very flat morphisms of commutative rings.

Lemma 9.3 Let $R$ be a commutative ring and $S$ be a commutative $R$-algebra. Then

(a) the $R$-algebra $S$ is very flat if and only if every very flat $S$-module is a very flat $R$-module;

(b) the $R$-algebra $S$ is finitely very flat if and only if every finitely very flat $S$-module is a finitely very flat $R$-module.

Proof Part (a): to prove the "if", it suffices to consider the very flat $S$-module $S\left[s^{-1}\right]$. The assertion "only if" is [22, Lemma 1.2.3(b)]. Part (b): once again, to prove the "if", it suffices to consider the finitely very flat $S$-module $S\left[s^{-1}\right]$. "Only if": let $F$ be a finitely very flat $S$-module, and let $\mathbf{s}=\left\{s_{1}, \ldots, s_{m}\right\}$ be a finite set of elements in $S$ such that the $S$-module $F$ is s-very flat. Set $s_{0}=1 \in S$, and for every $0 \leq j \leq m$ choose a finite set of elements $\mathbf{r}_{j}=\left\{r_{j, 1}, \ldots, r_{j, n_{j}}\right\} \subset R$ such that the $R$-module $S\left[s_{j}^{-1}\right]$ is $\mathbf{r}_{j}$-very flat. Denote by $\mathbf{r} \subset R$ the finite set of all elements $\left\{r_{j, k}\right\}, 0 \leq j \leq m, 1 \leq k \leq n_{j}$. Then the $R$-module $F$ is $\mathbf{r}$-very flat.

Example 9.4 This example, suggested to us by Jan Trlifaj, shows that the finite presentability condition on the $R$-algebra $S$ or the $S$-module $F$ cannot be replaced by a finite generatedness condition in the formulations of Main Theorems 1.1 and 1.2.

Let $R$ be a von Neumann regular commutative ring. Then all $R$-modules are flat, while all very flat $R$-modules are projective [29, Example 2.9]. Let $I \subset R$ be an infinitely generated ideal. Then $S=R / I$ is a finitely generated flat $R$-algebra which is not a very flat $R$-module. Alternatively, set $S=R$ and $F=R / I$; then, $S$ is a finitely presented $R$-algebra and $F$ is a finitely generated $S$-module which is a flat, but not a very flat $R$-module.

Main Theorem 1.2 tells, on the other hand, that if $R$ is a von Neumann regular commutative ring, $S$ is a finitely presented $R$-algebra, and $F$ is a finitely presented $S$-module, then $F$ is a projective $R$-module.

The following corollary is an application of Main Theorem 1.2 or 1.5.

Corollary 9.5 Let $R$ be a commutative ring, $P$ be a finitely generated projective $R$-module, and $x: P \longrightarrow P$ be an $R$-linear map. Denote by $P\left[x^{-1}\right]$ the inductive limit of the sequence of maps

$$
P \stackrel{x}{\longrightarrow} P \stackrel{x}{\longrightarrow} P \stackrel{x}{\longrightarrow} \cdots
$$

Then $P\left[x^{-1}\right]$ is a finitely very flat $R$-module.

Proof Consider the algebra of polynomials $R[x]$ in one variable $x$ over the ring $R$. Then $P$ can be viewed as an $R[x]$-module in which the element $x \in R[x]$ acts by the operator $x: P \longrightarrow P$. Notice that $P$ is a finitely presented $R[x]$-module.

Indeed, let $p_{1}, \ldots, p_{m} \in P$ be a set of generators of the $R$-module $P$. A finitely generated projective $R$-module is finitely presentable; specifically, if $f: R^{m} \longrightarrow P$ is the surjective map taking the free generator $e_{i} \in R^{m}$ to the generator $p_{i} \in P$ and $g: P \longrightarrow R^{m}$ is a splitting of the $R$-module morphism $f$, then $P$ is the cokernel of the $R$-module morphism 
$\operatorname{id}_{R^{m}}-g f: R^{m} \longrightarrow R^{m}$. So the elements $e_{i}-g f\left(e_{i}\right) \in R^{m}$ form a finite set of relations defining the $R$-module $P$ as a quotient $R$-module of the free $R$-module $R^{m}$. Now let $x_{i j} \in R$, $1 \leq i, j \leq m$ be some elements such that $x\left(p_{i}\right)=\sum_{j=1}^{m} x_{i j} p_{j}$ for all $1 \leq i \leq m$. Then the elements $e_{i}-g f\left(e_{i}\right) \in R^{m} \subset R[x]^{m}$ and $x e_{i}-\sum_{j=1}^{m} x_{i j} e_{j} \in R[x]^{m}$ can be used as a finite set of relations defining the $R[x]$-module $P$ as a quotient module of the free $R[x]$-module $R[x]^{m}$.

Denoting by $R\left[x, x^{-1}\right]$ the algebra of Laurent polynomials in the variable $x$ over the ring $R$, it follows that $P\left[x^{-1}\right] \simeq R\left[x, x^{-1}\right] \otimes_{R[x]} P$ is a finitely presented $R\left[x, x^{-1}\right]$-module. Clearly, $P\left[x^{-1}\right]$ is also a flat $R$-module, while $R\left[x, x^{-1}\right]$ is a finitely presented $R$-algebra. Applying Main Theorem 1.2 to the $R$-algebra $S=R\left[x, x^{-1}\right]$ and the $S$-module $F=P\left[x^{-1}\right]$, we conclude that $P\left[x^{-1}\right]$ is a very flat $R$-module. Applying Main Theorem 1.5 , we see that $P\left[x^{-1}\right]$ is even a finitely very flat $R$-module.

The above discussion of finite presentability of a finitely generated projective module could be avoided by replacing a projective $R$-module $P$ with an $R$-linear map $x: P \longrightarrow P$ by a direct sum $(P, x) \oplus(Q, y)$, where $Q$ is a finitely generated projective $R$-module such that the $R$-module $P \oplus Q$ is free, and $y: Q \longrightarrow Q$ is an arbitrary $R$-linear map. Then the $R$-module $P\left[x^{-1}\right]$ is a direct summand of $(P \oplus Q)\left[(x \oplus y)^{-1}\right]$, and the passage to a direct summand preserves (finite) very flatness. Taking $y=0$, one even gets $(P \oplus Q)\left[(x \oplus y)^{-1}\right]=P\left[x^{-1}\right]$. Denote the finitely generated free $R$-module $P \oplus Q$ by $R^{m}$ and the $R$-linear operator $x \oplus y$ by $z: R^{m} \longrightarrow R^{m}$. Then one can define the $R[x]$-module $R^{m}$, with the element $x \in R[x]$ acting in it by the operator $z$, by the finite set of relations $x e_{i}-\sum_{z_{i j} e_{j}} z_{i j} e_{j} \in R[x]^{m}$, where $e_{i} \in R^{m}, 1 \leq i \leq m$ are the free generators of the $R$-module $R^{m}$ and $z_{i j} \in R, 1 \leq i, j \leq m$ are the elements such that $z\left(e_{i}\right)=\sum_{j=1}^{m} z_{i j} e_{j}$ for all $1 \leq i \leq m$.

Alternatively, one could say that there is a subring $\bar{R} \subset R$ finitely generated over $\mathbb{Z}$ and a finitely generated projective $\bar{R}$-module $\bar{P}$ with an $\bar{R}$-linear map $\bar{x}: \bar{P} \longrightarrow \bar{P}$ such that $P=R \otimes_{\bar{R}} \bar{P}$ and $x=R \otimes_{\bar{R}} \bar{x}$ (because only a finite set of elements of $R$ are involved as the matrix entries of an idempotent linear operator $R^{m} \longrightarrow R^{m}$ defining $P$ and the operator $x: P \longrightarrow P$ ). Then $\bar{P}$ is a finitely generated module over a Noetherian $\operatorname{ring} \bar{R}[x]$ and $\bar{P}\left[\bar{x}^{-1}\right]$ is a finitely generated/presented module over $\bar{R}\left[x, x^{-1}\right]$, so one can apply Main Theorem 1.5 to the $\bar{R}$-algebra $\bar{R}\left[x, x^{-1}\right]$ and the $\bar{R}\left[x, x^{-1}\right]$-module $\bar{P}\left[\bar{x}^{-1}\right]$ in order to show that the $\bar{R}$-module $\bar{P}\left[\bar{x}^{-1}\right]$ is finitely very flat. Hence, the $R$-module $P\left[x^{-1}\right]=R \otimes_{R} \bar{P}\left[\bar{x}^{-1}\right]$ is finitely very flat.

Example 9.6 The following counterexample shows that the condition that the projective $R$ module $P$ should be finitely generated cannot be dropped in Corollary 9.5. Let $R=\mathbb{Z}$ be the ring of integers and $P$ be a free abelian group with an infinite set of generators $p_{1}, p_{2}$, $p_{3}, \ldots$ Let the additive operator $x: P \longrightarrow P$ act by the rule $x\left(p_{n}\right)=(n+1) p_{n+1}, n \geq 1$. Then the abelian group $P\left[x^{-1}\right]$ is a vector space of countable dimension over the field of rational numbers $\mathbb{Q}$, which is not a very flat $\mathbb{Z}$-module [22, Example 1.7.7].

Example 9.7 This is a counterexample showing that a commutative $R$-algebra $S$ can be a free $R$-module without being a very flat $R$-algebra (cf. Sect. 0.6). Once again, set $R=\mathbb{Z}$, and let $S$ be the "trivial ring extension" $S=\mathbb{Z}[x] \oplus P$, where $P$ is the abelian group from Example 9.6. The multiplication in $S$ is defined by the rule $(f, p)(g, q)=(f g, g(p)+f(q))$, where $f, g \in \mathbb{Z}[x], p, q \in P$, and the $\mathbb{Z}[x]$-module structure on $P$ is given by the operator $x: P \longrightarrow P$ from Example 9.6. This formula means that, by the definition, the product of any two elements of $P$ is zero in $S$. So $S$ is a free abelian group and a commutative $\mathbb{Z}$-algebra with an element $x \in S$, but $S\left[x^{-1}\right]=\mathbb{Z}\left[x, x^{-1}\right] \oplus P\left[x^{-1}\right]$ is not a very flat $\mathbb{Z}$-module.

Finally, let us prove the last property (VF16) announced in Sect. 0.7. 
Corollary 9.8 Let $k$ be a field, $R$ and $S$ be commutative $k$-algebras, and $t \in R \otimes_{k} S$ be an element. Then the $R$-module $\left(R \otimes_{k} S\right)\left[t^{-1}\right]$ is very flat.

Proof Notice that the assertion of the corollary can be generalized as follows. Let $M$ be an $S$-module. Then $R \otimes_{k} M$ is an $\left(R \otimes_{k} S\right)$-module, and one can consider the $\left(R \otimes_{k} S\right)\left[t^{-1}\right]$ module $\left(R \otimes_{k} M\right)\left[t^{-1}\right]=\left(R \otimes_{k} S\right)\left[t^{-1}\right] \otimes_{R \otimes_{k} S}\left(R \otimes_{k} M\right)$. We claim that $\left(R \otimes_{k} M\right)\left[t^{-1}\right]$ is a very flat $R$-module.

Let $r_{1}, \ldots, r_{m} \in R$ and $s_{1}, \ldots, s_{m} \in S$ be some elements such that $t=r_{1} \otimes s_{1}+$ $\cdots+r_{m} \otimes s_{m} \in R \otimes_{k} S$. Denote by $\bar{S}$ the $k$-subalgebra generated by $s_{1}, \ldots, s_{m}$ in $S$. Then $t \in R \otimes_{k} \bar{S} \subset R \otimes_{k} S$, and the $R$-module $\left(R \otimes_{k} M\right)\left[t^{-1}\right]$ only depends on the $\bar{S}$-module structure on $M$, rather than on the whole $S$-module structure. Replacing $S$ with $\bar{S}$, for any $\bar{S}$-module $M$ we can consider the $\left(R \otimes_{k} \bar{S}\right)\left[t^{-1}\right]$-module $\left(R \otimes_{k} M\right)\left[t^{-1}\right]$. Let us show that $\left(R \otimes_{k} M\right)\left[t^{-1}\right]$ is a very flat $R$-module for any $\bar{S}$-module $M$.

Any module over a commutative ring $\bar{S}$ is a transfinitely iterated extension, in the sense of the inductive limit, of the $\bar{S}$-modules $\bar{S} / I$, where $I \subset R$ are ideals. The functor $M \longmapsto$ $\left(R \otimes_{k} M\right)\left[t^{-1}\right]$ is exact and preserves inductive limits, so it preserves transfinitely iterated extensions. Thus the $\left(R \otimes_{k} \bar{S}\right)\left[t^{-1}\right]$-module $\left(R \otimes_{k} M\right)\left[t^{-1}\right]$ is a transfinitely iterated extension of the $\left(R \otimes_{k} \bar{S}\right)\left[t^{-1}\right]$-modules $\left(R \otimes_{k} \bar{S} / I\right)\left[t^{-1}\right]$.

It remains to show that the $R$-module $\left(R \otimes_{k} \bar{S} / I\right)\left[t^{-1}\right]$ is very flat for every ideal $I \subset \bar{S}$. Now $\bar{S} / I$ is a finitely generated $k$-algebra; hence, $R \otimes_{k} \bar{S} / I$ is a finitely presented $R$-algebra. Furthermore, $R \otimes_{k} \bar{S} / I$ is a free $R$-module. Applying Corollary 9.1, we conclude that $R \otimes_{k} \bar{S} / I$ is a very flat $R$-algebra, so the $R$-module $\left(R \otimes_{k} \bar{S} / I\right)\left[t^{-1}\right]$ is very flat. (In fact, the $R$-module $\left(R \otimes_{k} \bar{S} / I\right)\left[t^{-1}\right]$ is even finitely very flat, by Corollary 9.2 , but finite very flatness as such is not preserved by transfinitely iterated extensions, so the $R$-module $\left(R \otimes_{k} S\right)\left[t^{-1}\right]$ is still only very flat.)

\section{Descent for surjective morphisms}

The proofs of Theorems 1.11 and 1.12 are similar to (but slightly more complicated than) the argument deducing the main theorems from the main lemmas in Sect. 2.

The most important technical property of the class of all morphisms of commutative rings inducing surjective maps of the spectra is its stability under the base change. Let us start with the following trivial observation: the spectrum of a commutative ring $R$ is empty if and only if $R=0$.

Lemma 10.1 Let $R \longrightarrow S$ be a homomorphism of commutative rings such that the induced map of the spectra $\operatorname{Spec} S \longrightarrow \operatorname{Spec} R$ is surjective. Let $R \longrightarrow R^{\prime}$ be an arbitrary morphism of commutative rings. Then the map $\operatorname{Spec}\left(R^{\prime} \otimes_{R} S\right) \longrightarrow \operatorname{Spec} R^{\prime}$ induced by the homomorphism of commutative rings $R^{\prime} \longrightarrow R^{\prime} \otimes_{R} S$ is surjective.

Proof Let $\mathfrak{p}^{\prime}$ be a prime ideal in the ring $R^{\prime}$ and $\mathfrak{p}$ be its image under the map Spec $R^{\prime} \longrightarrow$ Spec $R$. By assumption, there exists a prime ideal $\mathfrak{q}$ in the $\operatorname{ring} S$ whose image under the map Spec $S \longrightarrow$ Spec $R$ is equal to $\mathfrak{p}$. Let $k_{\mathfrak{p}^{\prime}}\left(R^{\prime}\right), k_{\mathfrak{p}}(R)$, and $k_{\mathfrak{q}}(S)$ denote the residue fields of the prime ideals $\mathfrak{p}^{\prime} \subset R^{\prime}, \mathfrak{p} \subset R$, and $\mathfrak{q} \subset S$. Then the ring $k_{\mathfrak{p}^{\prime}}\left(R^{\prime}\right) \otimes_{k_{\mathfrak{p}}(R)} k_{\mathfrak{q}}(S)$ is nonzero (as the tensor product of nonzero vector spaces over a field), so it has a maximal ideal $\mathfrak{m} \subset$ $k_{\mathfrak{p}^{\prime}}\left(R^{\prime}\right) \otimes_{k_{\mathfrak{p}}(R)} k_{\mathfrak{q}}(S)$. There is a natural ring homomorphism $R^{\prime} \otimes_{R} S \longrightarrow k_{\mathfrak{p}^{\prime}}\left(R^{\prime}\right) \otimes_{k_{\mathfrak{p}}(R)}$ $k_{\mathfrak{q}}(S)$; denote the full preimage of $\mathfrak{m}$ under this ring homomorphism by $\mathfrak{q}^{\prime} \subset R^{\prime} \otimes_{R} S$. Then $\mathfrak{q}^{\prime} \in \operatorname{Spec}\left(R^{\prime} \otimes_{R} S\right)$ is a point whose image under the map $\operatorname{Spec}\left(R^{\prime} \otimes_{R} S\right) \longrightarrow \operatorname{Spec} R^{\prime}$ is equal to $\mathfrak{p}^{\prime}$. 
Let $R$ be a commutative ring. A projective $R$-module $P$ is said to be faithfully projective if it is a projective generator of the abelian category $R$-mod, that is $\operatorname{Hom}_{R}(P, M)=0$ implies $M=0$ for any $R$-module $M$. Equivalently, it means that the free $R$-module $R$ is a direct summand of a (finite) direct sum of copies of $P$.

Lemma 10.2 Let $R$ be a commutative ring and $S$ be a commutative $R$-algebra such that $S$ is a faithfully projective $R$-module. Let $F$ be an $R$-module.

(a) Assume that the R-algebra $S$ is very flat. Then the $R$-module $F$ is very flat if and only if the $S$-module $S \otimes_{R} F$ is very flat.

(b) Assume that the $R$-algebra $S$ is finitely very flat. Then the $R$-module $F$ is finitely very flat if and only if the $S$-module $S \otimes_{R} F$ is finitely very flat.

Proof This is a version of [22, Lemma 1.7.12]. Part (a): the "only if" holds by Lemma 2.2(a). To prove the "if", recall that all the very flat $S$-modules are very flat $R$-modules by Lemma 9.3(a). In particular, the very flat $S$-module $S \otimes_{R} F$ is a very flat $R$-module. It remains to use the assumption that the $R$-module $R$ is a direct summand of a finite direct sum of copies of the $R$-module $S$, so the $R$-module $F$ is a direct summand of a finite direct sum of copies of the $R$-module $S \otimes_{R} F$.

Part (b): the "only if" holds by Lemma 2.2(b). To prove the "if", recall that all the finitely very flat $S$-modules are finitely very flat $R$-modules by Lemma 9.3(b). In particular, the finitely very flat $S$-module $S \otimes_{R} F$ is a finitely very flat $R$-module. The argument finishes in the same way as the proof of part (a).

Lemma 10.3 Let $R$ be a commutative ring and $S$ be a finitely presented commutative $R$ algebra such that $S$ is a faithfully projective $R$-module. Let $F$ be an $R$-module. Then

(a) the $R$-module $F$ is very flat if and only if the $S$-module $S \otimes_{R} F$ is very flat;

(b) the $R$-module $F$ is finitely very flat if and only if the $S$-module $S \otimes_{R} F$ is finitely very flat.

Proof Part (a): the $R$-algebra $S$ is very flat by Corollary 9.1, so Lemma 10.2(a) can be applied. Part (b): the $R$-algebra $S$ is finitely very flat by Corollary 9.2, so Lemma 10.2(b) can be applied.

The following proposition is a particular case of (the "if" assertion of) Theorem 1.11 from which the general case is deduced.

Proposition 10.4 Let $R$ be a Noetherian commutative ring and $S$ be a finitely generated commutative $R$-algebra such that the induced map of the spectra $\operatorname{Spec} S \longrightarrow \operatorname{Spec} R$ is surjective. Let $F$ be a flat $R$-module. Assume that the $S$-module $S \otimes_{R} F$ is very flat, and the $R / r R$-module $F / r F$ is very flat for every nonzero element $r \in R$. Then $F$ is a very flat $R$-module.

Proof Case I: the ring $R$ contains zero-divisors. Let $a \neq 0 \neq b$ be two elements in $R$ such that $a b=0$ in $R$. By assumption, $F / a F$ is a very flat $R / a R$-module and $F / b F$ is a very flat $R / b R$-module. As in the proof of Proposition 2.3, it follows from the latter that $F\left[a^{-1}\right]$ is a very flat $R\left[a^{-1}\right]$-module. By Main Lemma 1.4, we conclude that $F$ is a very flat $R$-module.

Case II: the ring $R$ is an integral domain. By Lemma 2.1, there exists a nonzero element $a \in R$ such that the $R\left[a^{-1}\right]$-module $S\left[a^{-1}\right]$ is free. By Lemma 10.1, the map of spectra Spec $S\left[a^{-1}\right] \longrightarrow \operatorname{Spec} R\left[a^{-1}\right]$ is surjective, so the ring $S\left[a^{-1}\right]$ is nonzero whenever the ring $R\left[a^{-1}\right]$ is nonzero. Hence, $S\left[a^{-1}\right]$ is a faithfully projective $R\left[a^{-1}\right]$-module. The 
$S\left[a^{-1}\right]$-module $S\left[a^{-1}\right] \otimes_{R} F$ is very flat, since the $S$-module $S \otimes_{R} F$ is very flat. Applying Lemma 10.3 (a) to the ring $R\left[a^{-1}\right]$, the algebra $S\left[a^{-1}\right]$ over it, and the module $F\left[a^{-1}\right]$, we conclude that the $R\left[a^{-1}\right]$-module $F\left[a^{-1}\right]$ is very flat.

By assumption, the $R / a R$-module $F / a F$ is very flat. By Main Lemma 1.4, it follows that $F$ is a very flat $R$-module.

Proof of Theorem 1.11 The assertion "only if" is a particular case of Lemma 2.2(a). The assertion "if" is the nontrivial part.

Assume that the $R$-module $F$ is not very flat. By Proposition 10.4, it follows that there exists a nonzero element $r \in R$ such that the $R / r R$-module $F / r F$ is not very flat. Now $R_{1}=R / r R$ is a Noetherian commutative ring, $S_{1}=S / r S$ is a finitely generated commutative $R_{1}$-algebra, and the map Spec $S_{1} \longrightarrow \operatorname{Spec} R_{1}$ is surjective by Lemma 10.1. Furthermore, $F_{1}=F / r F$ is a flat $R / r R$-module.

So Proposition 10.4 can be applied again in order to produce a nonzero element $r_{1} \in R_{1}$ such that the $R_{1} / r_{1} R_{1}$-module $F_{1} / r_{1} F_{1}$ is not very flat, etc. The argument finishes in the same way as the proof of Main Theorem 1.3 in Sect. 2.

Similarly, the next proposition is a particular case of Theorem 1.12 from which the general case is deduced.

Proposition 10.5 Let $\bar{R}$ be a Noetherian commutative ring and $\bar{S}$ be a finitely generated commutative $\bar{R}$-algebra. Let $R$ be a commutative $\bar{R}$-algebra; set $S=R \otimes_{\bar{R}} \bar{S}$ and assume that the induced map of spectra Spec $S \longrightarrow \operatorname{Spec} R$ is surjective. Let $F$ be a flat $R$-module. Assume that the $S$-module $S \otimes_{R} F$ is finitely very flat, and the $R / r R$-module $F / r F$ is finitely very flat for every nonzero element $r \in \bar{R}$. Then $F$ is a finitely very flat $R$-module.

Proof Case I: the ring $\bar{R}$ contains zero-divisors. The argument in this case is similar to the proofs of Case I in Propositions 2.6 and 10.4. It does not use the rings $\bar{S}$ and $S$ and the assumptions related to them (but uses Lemma 2.2(b) and Main Lemma 1.6).

Case II: the ring $\bar{R}$ is an integral domain. By Lemma 2.1, there exists a nonzero element $a \in \bar{R}$ such that the $\bar{R}\left[a^{-1}\right]$-module $\bar{S}$ is free. Then the $R\left[a^{-1}\right]$-module $S\left[a^{-1}\right]$ is free, too. Arguing as in the proof of Proposition 10.4, we see that $S\left[a^{-1}\right]$ is a faithfully projective $R\left[a^{-1}\right]$-module. The $S\left[a^{-1}\right]$-module $S\left[a^{-1}\right] \otimes_{R} F$ is finitely very flat, since the $S$-module $S \otimes_{R} F$ is finitely very flat. Applying Lemma 10.3(b), we conclude that the $R\left[a^{-1}\right]$-module $F\left[a^{-1}\right]$ is finitely very flat, and the argument finishes as in the proof of Proposition 10.4, using Main Lemma 1.6.

Proof of Theorem 1.12 The assertion "only if" is a particular case of Lemma 2.2(b). "If" is the nontrivial part. First of all, we apply Lemma 2.4 in order to produce a Noetherian commutative ring $\bar{R}$ with a ring homomorphism $\bar{R} \longrightarrow R$ and a finitely generated commutative $\bar{R}$-algebra $\bar{S}$ such that $S=R \otimes_{\bar{R}} \bar{S}$.

Assume that the $R$-module $F$ is not finitely very flat. By Proposition 10.5, it then follows that there exists a nonzero element $r \in \bar{R}$ such that the $R / r R$-module $F / r F$ is not finitely very flat. The argument finishes in the way similar to the proof of Main Theorem 1.5 in Sect. 2 and the above proof of Theorem 1.11.

Let $R$ be a commutative ring. A flat $R$-module $F$ is said to be faithfully flat if $F \otimes_{R} N=0$ implies $N=0$ for any $R$-module $N$. The $P$-support $\operatorname{PSupp}_{R} M \subset \operatorname{Spec} R$ of an $R$-module $M$ is the set of all prime ideals $\mathfrak{p} \subset R$ such that $k_{\mathfrak{p}}(R) \otimes_{R} M \neq 0$, where $k_{\mathfrak{p}}(R)$ denotes the residue field of the prime ideal $\mathfrak{p}$ in $R$ (cf. Sect. 0.3, [22, Section 1.7], and [29, Definition 2.7]). 
A flat $R$-module $F$ is faithfully flat if and only if its $\mathrm{P}$-support $\operatorname{PSupp}_{R} F$ coincides with the whole of Spec $R$.

An $R$-algebra is said to be faithfully flat if it is faithfully flat as an $R$-module. A flat commutative $R$-algebra $S$ is faithfully flat if and only if the induced map of the spectra Spec $S \longrightarrow$ Spec $R$ is surjective.

Lemma 10.6 Let $R$ be a commutative ring and $S$ be a faithfully flat commutative $R$-algebra. Then an $R$-module $F$ is flat if and only if the $S$-module $S \otimes_{R} F$ is flat.

Proof The "only if" holds because flatness of modules is preserved by extensions of scalars. To prove the "if", notice the isomorphisms $S \otimes_{R} \operatorname{Tor}_{i}^{R}(F, N) \simeq \operatorname{Tor}_{i}^{R}\left(S \otimes_{R} F, N\right) \simeq$ $\operatorname{Tor}_{i}^{S}\left(S \otimes_{R} F, S \otimes_{R} N\right)$, which hold for any flat $R$-algebra $S$, any $R$-modules $F$ and $N$, and all $i \geq 0$. In particular, if $S$ is a faithfully flat $R$-algebra, then $\operatorname{Tor}_{1}^{S}\left(S \otimes_{R} F, S \otimes_{R} N\right)=0$ implies $\operatorname{Tor}_{1}^{R}(F, N)=0$.

Corollary 10.7 Let $R$ be a Noetherian commutative ring and $S$ be a finitely generated faithfully flat commutative $R$-algebra. Then an $R$-module $F$ is very flat if and only if the $S$-module $S \otimes_{R} F$ is very flat.

Proof The "only if" holds by Lemma 2.2(a). To prove the "if", assume that $F$ is an $R$-module such that the $S$-module $S \otimes_{R} F$ is very flat. Then, in particular, the $S$-module $S \otimes_{R} F$ is flat; hence, by Lemma 10.6 the $R$-module $F$ is flat. Now we can apply Theorem 1.11 in order to conclude that the $R$-module $F$ is very flat.

Corollary 10.8 Let $R$ be a commutative ring and $S$ be a finitely presented faithfully flat commutative $R$-algebra. Then an $R$-module $F$ is finitely very flat if and only if the $S$-module $S \otimes_{R} F$ is finitely very flat.

Proof Similar to the proof of Corollary 10.7, using Lemma 2.2(b) (for the "only if") and Lemma 10.6 with Theorem 1.12 (for the "if").

Notice that Corollary 10.8 is a generalization of Lemma 10.3(b), but Corollary 10.7 does not imply Lemma 10.3(a) [because there is a Noetherianity assumption in Corollary 10.7, which was not needed in Lemma 10.3(a)].

Acknowledgements This paper grew out of the first author's visits to Prague in 2015-2017, and he wishes to thank to Jan Trlifaj for inviting him. Our discussions with Jan Trlifaj also played a particularly important role in the development of this project. The first author is grateful to Silvana Bazzoni, Jan Št ovíček, and Amnon Yekutieli for helpful conversations. The first author's research is supported by research plan RVO: 67985840, by the Israel Science Foundation Grant \# 446/15, and by the Grant Agency of the Czech Republic under the Grant P201/12/G028. The second author's research is supported by the Grant Agency of the Czech Republic under the Grant 17-23112S and by the SVV project under the Grant SVV-2017-260456.

\section{References}

1. Bass, H.: Big projective modules are free. Ill. J. Math. 7(1), 24-31 (1963)

2. Bazzoni, S., Salce, L.: Strongly flat covers. J. Lond. Math. Soc. 66(2), 276-294 (2002)

3. Bazzoni, S., Salce, L.: On strongly flat modules over integral domains. Rocky Mt. J. Math. 34(2), 417-439 (2004)

4. Bican, L., El Bashir, R., Enochs, E.: All modules have flat covers. Bull. Lond. Math. Soc. 33(4), 385-390 (2001)

5. Efimov, A.I., Positselski, L.: Coherent analogues of matrix factorizations and relative singularity categories. Algebra Number Theory 9(5), 1159-1292 (2015). arXiv:1102.0261 [math.CT] 
6. Eklof, P.C., Trlifaj, J.: How to make Ext vanish. Bull. Lond. Math. Soc. 33(1), 41-51 (2001)

7. Enochs, E.: Flat covers and flat cotorsion modules. Proc. Am. Math. Soc. 92(2), 179-184 (1984)

8. Estrada, S., Slávik, A.: Quillen equivalent models for the derived category of flats and the resolution property. Electronic preprint arXiv:1708.05913 [math.AT]

9. Geigle, W., Lenzing, H.: Perpendicular categories with applications to representations and sheaves. J. Algebra 144(2), 273-343 (1991)

10. Göbel, R., Trlifaj, J.: Approximations and Endomorphism Algebras of Modules. Second Revised and Extended Edition. De Gruyter Expositions in Mathematics 41. De Gruyter, Berlin (2012)

11. Grothendieck, A., Dieudonné, J.: Éléments de géométrie algébrique IV (1965) Étude locale des schémas et des morphismes des schémas, Seconde partie. Publications Mathematiques de l'IHÉS 24, 5-231 (1965)

12. Matsumura, H.: Commutative Ring Theory. Translated by M. Reid. Cambridge University Press, Cambridge (1986-2006)

13. Murfet, D.: Derived categories of quasi-coherent sheaves. Notes (Oct 2006). http://www.therisingsea.org/ notes. Accessed 24 Aug 2019

14. Murfet, D.: The mock homotopy category of projectives and Grothendieck duality. Ph.D. Thesis, Australian National University (Sept 2007). http://www.therisingsea.org/thesis.pdf. Accessed 24 Aug 2019

15. Neeman, A.: The homotopy category of flat modules, and Grothendieck duality. Invent. Math. 174, 225308 (2008)

16. Orlov, D.: Matrix factorizations for nonaffine LG-models. Math. Ann. 353(1), 95-108 (2012). arXiv:1101.4051 [math.AG]

17. Perry, A.: Faithfully flat descent for projectivity of modules. Electronic preprint arXiv:1011.0038 [math.AC]

18. Porta, M., Shaul, L., Yekutieli, A.: On the homology of completion and torsion. Algebras Represent. Theory 17(1), 31-67 (2014). arXiv:1010.4386 [math.AC]. Erratum in Algebras and Represent. Theory 18(5), 1401-1405 (2015). arXiv:1506.07765 [math.AC]

19. Positselski, L.: Homological Algebra of Semimodules and Semicontramodules: Semi-infinite Homological Algebra of Associative Algebraic Structures. Appendix C in collaboration with D. Rumynin; Appendix D in collaboration with S. Arkhipov. Monografie Matematyczne, vol. 70. Birkhäuser/Springer, Basel (2010). xxiv+349 pp. arXiv:0708.3398 [math.CT]

20. Positselski, L.: Two kinds of derived categories, Koszul duality, and comodule-contramodule correspondence. Mem. Am. Math. Soc. 212(996), vi+133 pp (2011). arXiv:0905.2621 [math.CT]

21. Positselski, L.: Weakly curved $\mathrm{A}_{\infty}$-algebras over a topological local ring. Mémoires de la Société Mathématique de France 159, vi+206 pp (2018). arXiv:1202.2697 [math.CT]

22. Positselski, L.: Contraherent cosheaves. Electronic preprint arXiv:1209.2995 [math.CT]

23. Positselski, L.: Dedualizing complexes and MGM duality. J. Pure Appl. Algebra 220(12), 3866-3909 (2016). arXiv:1503.05523 [math.CT]

24. Positselski, L.: Contraadjusted modules, contramodules, and reduced cotorsion modules. Mosc. Math. J. 17(3), 385-455 (2017). arXiv:1605.03934 [math.CT]

25. Positselski, L.: Abelian right perpendicular subcategories in module categories. Electronic preprint arXiv:1705.04960 [math.CT]

26. Positselski, L., Rosický, J.: Covers, envelopes, and cotorsion theories in locally presentable abelian categories and contramodule categories. J. Algebra 483, 83-128 (2017). arXiv:1512.08119 [math.CT]

27. Positselski, L., Slávik, A.: On strongly flat and weakly cotorsion modules. Math. Z. 291(3-4), 831-875 (2019). arXiv:1708.06833 [math.AC]

28. Raynaud, M., Gruson, L.: Critères de platitude et de projectivité: Techniques de "platification" d'un module. Invent. Math. 13(1-2), 1-89 (1971)

29. Slávik, A., Trlifaj, J.: Very flat, locally very flat, andcontraadjusted modules. J. Pure Appl. Algebra 220(12), 3910-3926 (2016). arXiv:1601.00783 [math.AC]

30. Št'ovíček, J.: Exact model categories, approximation theory, and cohomology of quasi-coherent sheaves. In: Advances in Representation Theory of Algebras. EMS Series of Congress Reports, European Mathematical Society, Zürich, pp. 297-367 (2013). arXiv:1301.5206 [math.CT]

31. Totaro, B.: The resolution property for schemes and stacks. J. für die reine und angew. Mathematik 577, 1-22 (2004). arXiv:math.AG/0207210

32. Yekutieli, A.: A separated cohomologically complete module is complete. Commun. Algebra 43(2), 616-622 (2015). arXiv:1312.2714 [math.AC]

33. Yekutieli, A.: Flatness and completion revisited. Algebras Represent. Theory 21(4), 717-736 (2018). arXiv:1606.01832 [math.AC]

Publisher's Note Springer Nature remains neutral with regard to jurisdictional claims in published maps and institutional affiliations. 